\title{
The quark anti-quark potential and the cusp anomalous dimension from a TBA equation
}

\author{
Diego Correa, ${ }^{a}$ Juan Maldacena ${ }^{b}$ and Amit Sever ${ }^{b, c}$ \\ ${ }^{a}$ Instituto de Física La Plata, Universidad Nacional de La Plata, \\ C.C. 67, 1900 La Plata, Argentina \\ ${ }^{b}$ School of Natural Sciences, Institute for Advanced Study, \\ Princeton, NJ 08540, U.S.A. \\ ${ }^{c}$ Perimeter Institute for Theoretical Physics \\ Waterloo, Ontario N2J 2W9, Canada \\ E-mail: diegocorrea@gmail.com, malda@ias.edu, amit.sever@gmail.com
}

ABSTRACT: We derive a set of integral equations of the TBA type for the generalized cusp anomalous dimension, or the quark antiquark potential on the three sphere, as a function of the angles. We do this by considering a family of local operators on a Wilson loop with charge $L$. In the large $L$ limit the problem can be solved in terms of a certain boundary reflection matrix. We determine this reflection matrix by using the symmetries and the boundary crossing equation. The cusp is introduced through a relative rotation between the two boundaries. Then the TBA trick of exchanging space and time leads to an exact equation for all values of $L$. The $L=0$ case corresponds to the cusped Wilson loop with no operators inserted. We then derive a slightly simplified integral equation which describes the small angle limit. We solve this equation up to three loops in perturbation theory and match the results that were obtained with more direct approaches.

Keywords: Wilson, 't Hooft and Polyakov loops, AdS-CFT Correspondence, Integrable Field Theories, Scattering Amplitudes

ARXIV EPRINT: 1203.1913 


\section{Contents}

1 Introduction $\quad \mathbf{2}$

1.1 Remarks on the cusp anomalous dimension 3

1.2 Method 4

2 Spectrum of operators on a Wilson line 7

2.1 The boundary reflection matrix 8

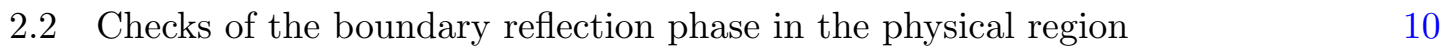

$\begin{array}{lll}2.3 & \text { Reflection matrix for a Wilson line at general angles } & 12\end{array}$

$\begin{array}{lll}2.4 & \text { Luscher computations and checks in the mirror region } & 12\end{array}$

$\begin{array}{ll}\text { 2.4.1 Leading Luscher correction at weak coupling } & 16\end{array}$

$\begin{array}{ll}\text { 2.4.2 Leading Luscher correction at strong coupling } & 17\end{array}$

3 The open Asymptotic Bethe Ansatz equations $\quad 17$

4 The boundary TBA equations $\quad 21$

4.1 Recovering the Luscher result 23

5 The near BPS limit $\quad 24$

5.1 The simplified equations at small angles 24

5.2 Weak coupling expansion of the small $\phi$ TBA 26

6 Conclusions and discussion $\quad 27$

$\begin{array}{lr}\text { A Reflection matrix } & 29\end{array}$

B Solution to the boundary crossing equation $\quad 30$

C Luscher correction at strong coupling $\quad 33$

C.1 Strong coupling expansion of the function $F \quad 35$

$\begin{array}{ll}\text { D Evaluating } t(q) & 36\end{array}$

$\begin{array}{ll}\text { E Derivation of the BTBA equations } & 38\end{array}$

E.1 Derivation by embedding into the bulk system 38

E.2 Direct derivation of the boundary TBA 43

E.3 Kernels conventions 46

$\begin{array}{lll}\text { F Perturbative solution of the small } \phi \text { TBA } & 47\end{array}$

$\begin{array}{lll}\text { F.1 Small } \phi \text { solution at 2-loops } & 48\end{array}$

F.2 Small $\phi$ solution at 3-loops 49

F.3 Recurrence resolvent 52 


\section{Introduction}

In this article we derive an equation for the cusp anomalous dimension for all angles and for all values of the 't Hooft coupling $\lambda=g_{Y M}^{2} N$ in the planar limit of $\mathcal{N}=4$ super Yang Mills. We obtain a system of non-linear integral equations of the form of a Thermodynamic Bethe Anstaz (TBA) system. The value of the cusp anomalous dimension can be obtained from a solution of the TBA system. This is also equal to the quark/anti-quark potential on the three sphere, see figure 1.

The cusp anomalous dimension is associated with the logarithmic divergence arising from a Wilson loop with a cusped contour [1]

$$
\langle W\rangle \sim e^{-\Gamma_{\text {cusp }}(\phi, \lambda) \log \frac{L_{\mathrm{IR}}}{\epsilon_{\mathrm{UV}}}},
$$

where $L_{\mathrm{IR}}$ and $\epsilon_{\mathrm{UV}}$ are IR and UV cutoffs respectively.

The locally supersymmetric Wilson loop in $\mathcal{N}=4$ super Yang Mills also includes a coupling to the scalar fields specified by a direction in the internal space $\vec{n}$ (with $\vec{n}^{2}=1$ )

$$
W \sim \operatorname{Tr}\left[P e^{i \oint A \cdot d x+\oint|d x| \vec{n} \cdot \vec{\Phi}}\right] .
$$

Instead of considering the same vector $\vec{n}$ on the two lines that make the cusp, we can take two vectors $\vec{n}$ and $\vec{n}^{\prime}$. This introduces a second angle $\cos \theta=\vec{n} \cdot \vec{n}^{\prime}$. Thus we have the generalized cusp anomalous dimension $\Gamma_{\text {cusp }}(\phi, \theta, \lambda)[2] . \quad \Gamma_{\text {cusp }}(\phi, \theta)$ can be computed in terms of a solution of the TBA system of equations presented in this article. We can also consider the continuation $\phi=i \varphi$, where $\varphi$ is a boost angle in Lorentzian signature. Before describing the computation, let us make some general remarks.

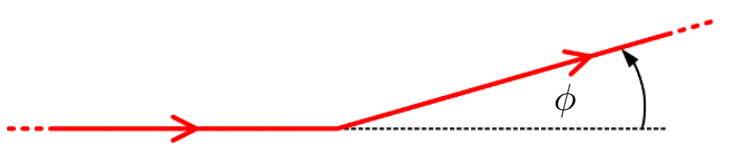

(a)

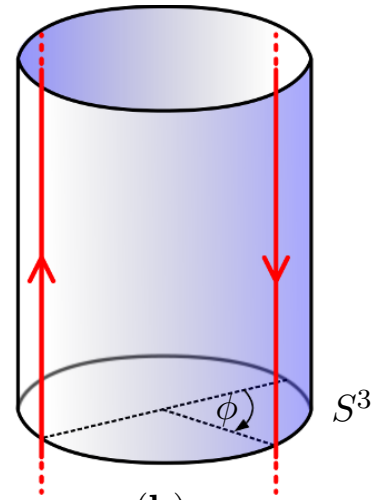

(b)

Figure 1. (a) A Wilson line with a cusp angle $\phi$. (b) Under the plane to cylinder map the two half lines in (a) are mapped to a quark anti-quark pair sitting at two points on $S^{3}$ at a relative angle of $\pi-\phi$. The quark anti-quark lines are extended along the time direction. 


\subsection{Remarks on the cusp anomalous dimension}

$\Gamma_{\text {cusp }}$ is related to a variety of physical observables:

- It characterizes the IR divergences that arise when we scatter massive colored particles. Here $\varphi$ is the boost angle between two external massive particle lines. For each consecutive pair of lines in the color ordered diagram we get a factor of the form (1.1), where $L_{I R}$ is the IR cutoff and $\epsilon_{\mathrm{UV}}^{2}$ is the given by the square of the sum of the momenta of the two consecutive particles. More explicitly, the angle is given by $\cosh \varphi=-\frac{p_{1} \cdot p_{2}}{\sqrt{p_{1}^{2} p_{2}^{2}}}$. This relation is general for any conformal gauge theory. See $[3,4]$ and references therein. In $\mathcal{N}=4$ super Yang Mills the massive particles can be obtained by setting some Higgs vevs to be non-zero $\vec{\Phi}$. Then the angle $\theta$ is the angle between the Higgs vevs associated to consecutive massive particles [5].

- The IR divergences of massless particles are characterized by $\Gamma_{\text {cusp }}^{\infty}$ which is the coefficient of the large $\varphi$ behavior of the cusp anomalous dimension, $\Gamma_{\text {cusp }} \propto \varphi \Gamma_{\text {cusp }}^{\infty}$. $\Gamma_{\text {cusp }}^{\infty}$ was computed in the seminal paper [6]. Note that $\Gamma_{\text {cusp }}^{\infty}$ is also sometimes called the "cusp anomalous dimension" though it is a particular limit of the general, angle dependent "cusp anomalous dimension" defined in (1.1) .

- By the plane to cylinder map this quantity is identical with the energy of a static quark and anti-quark sitting on a spatial three sphere at an angle $\pi-\phi$.

$$
\Gamma_{\text {cusp }}(\phi, \theta)=V(\phi, \theta) .
$$

See figure 1 . This potential depends on the angle $\phi$ as well as on the internal orientations of the quark and anti-quark, which define the second angle $\theta$.

- In particular, in the small $\delta=\pi-\phi$ limit we get the same answer as the quark-antiquark potential in flat space ${ }^{1}$

$$
\Gamma_{\text {cusp }}(\phi, \lambda) \sim \frac{v(\theta, \lambda)}{\delta}, \quad \text { when } \quad \delta=\pi-\phi \rightarrow 0,
$$

where $v(\lambda)$ is the coefficient of the quark-anti-quark potential, $V=\frac{v(\theta, \lambda)}{r}$, for a quark and an anti-quark at distance $r$ in flat space and couplings to the Higgs fields which are rotated by a relative angle $\theta$.

- In the small $\phi$ limit the cusp anomalous dimension goes as $\phi^{2}$ and one can define a Bremsstrahlung function $B$ by

$$
\Gamma_{\text {cusp }} \sim-\left(\phi^{2}-\theta^{2}\right) B(\lambda) \quad \phi, \theta \ll 1 .
$$

This function $B$ can be computed exactly using localization, see [8] and [9]. Here we will derive a set of integral equations that also determines $B$. In this way we can link the localization and integrability exact solutions. This function $B$ is also related to a variety of observables, see $[8,9]$ for further discussion.

\footnotetext{
${ }^{1}$ This limit does not commute with the perturbative expansion in $\lambda$. So (1.4) is correct if $\delta \ll \lambda$. If we expand first in $\lambda$ and then take the $\delta \rightarrow 0$ limit we get a different answer due to IR divergences that arise in the naive perturbative expansion. These also arise in QCD, The origin of these logs are discussed in [5, 7].
} 
Another motivation to study the cusp anomalous dimension is the study of amplitudes. Amplitudes are also functions of the angles between particles. Here we get a very simple function of one angle which has a structure very similar to amplitudes, since it is related to amplitudes of massive particles. Thus, obtaining exact results for this quantity is useful to learn about the general structure of the amplitude problem.

\subsection{Method}

The method to obtain the equation is a bit indirect and we need several preliminary results that are interesting in their own right. Just for orientation we will outline the main idea and method for its derivation.

The method consists of the following steps

- We first consider the problem of computing the spectrum of local operators on a Wilson line. We consider the particular case of operators with a large charge, i.e. operators containing a large number, $L$, of the complex scalar field $Z$ insertions. These insertions create a BMN vacuum [10].

- In the large $L$ limit the problem can be solved using an asymptotic Bethe Ansatz that involves the propagation of certain "magnons". These equations describe magnons moving on a long strip of length $L$ with two boundaries associated to the Wilson loop on each of the "sides" of the operator, see [11]. The propagation of the magnons in the bulk is the usual one [12]. The new feature is the existence of a boundary. The magnons are reflected at the boundary and one needs the boundary reflection matrix. This is fixed in two steps.

- We determine the matrix structure of the reflection matrix from group theory, as in [13-16]. This reflection matrix is such that it obeys the boundary Yang Baxter equation [17]. This is evidence that the boundary condition preserves integrability.

- We derive a crossing equation for the reflection phase and we find a solution.

- Doing a time/space flip, so that now we have the mirror theory between two boundary states separated by a mirror "time" $L$. See figure 2 . We can apply a symmetry generator that rotates one boundary relative to the other, so that we introduce the two angles.

- We compute this overlap using TBA equations for any $L$, focusing on the ground state energy, which is extracted by taking the large $T$ limit of the computation in figure 2. These boundary TBA equations can be derived following a method similar to the relativistic case [18].

- We set $L=0$ we get the cusp anomalous dimension.

Let us discuss these steps in a bit more detail. First we should note that the exact integrability methods, as currently understood, work best to compute energies of states. 
Thus, we should phrase the computation of the cusp anomalous dimension as the computation of an energy. This is very simple. Under the usual plane to cylinder map, the cusp on the plane maps into two static quark and anti-quark lines on $S^{3} \times R$. The quark and anti-quark lines are extended along the time direction, and they are separated by an angle $\pi-\phi$ on the $S^{3}$, see figure 1 . The case $\phi=0$, which is the straight line in the plane, is mapped to a quark-anti-quark pair at opposite points on the sphere. If $\theta=0$, this is a BPS configuration and the cusp anomalous dimension vanishes exactly for all $\lambda$. In fact, for $\theta= \pm \phi$ we continue to have a BPS configuration [19] and the cusp anomalous dimension continues to vanish. In general, the cusp anomalous dimension is the energy of this quark-anti-quark configuration, as a function of the two angles, $\phi$ and $\theta$.

The configuration with $\theta=\phi=0$ preserves 16 supercharges which, together with the bosonic symmetries, give rise to a $O S p\left(4^{*} \mid 4\right)$ symmetry group. This is important to determine the boundary reflection matrix.

Let us begin by considering an apparently unrelated problem which is the problem of computing the anomalous dimension of operators inserted along a Wilson loop. First we consider a straight Wilson loop and we insert an operator at the point $t=0$. For example, we can consider an insertion of a complex scalar field $Z$ on the contour

$$
P e^{i \int_{-\infty}^{0}\left(A+i \Phi_{4}\right)} Z(0) e^{i \int_{0}^{\infty}\left(A_{t}+i \Phi_{4}\right)}=B_{l} Z(0) B_{r}
$$

These are operators that live on the loop and should not be confused with closed string operators. We denote these operators as $B_{l} Z B_{r}$, where $B_{l, r}$ stands for the usual path ordered exponentials of the gauge field. The operator considered above is BPS if $Z$ is constructed out of scalars that do not appear in $B_{l, r}$ (1.6). To be definite, we consider $Z=\Phi_{5}+i \Phi_{6}$. We can similarly consider operators of the form $B_{l} Z^{L} B_{r}$ which continue to be BPS. The straight Wilson loop is invariant under dilatations, so we can characterize the operators by their dimension under dilatations. These operators have dimension $\Delta=L$.

Determining the scaling dimension of operators of this type, but with more general insertions, is easier in the large $L$ limit. Then, we can solve this problem by considering impurities propagating along a long chain of $Z$ 's. The impurities are the same as the ones that were used to solve the closed string problem in a similar regime [13, 20]. The new aspect is that the impurities can be reflected from the boundaries at the end of the chain. This picture was discussed at the 1-loop order in the weak coupling limit in [11]. To proceed, we need to determine the boundary reflection matrix to all orders in the coupling. The matrix structure can be determined by group theory, as in [13]. The phase factor is more subtle. We write a crossing equation for it and we solve it following the strategy outlined in $[21,22]$. At this stage we have completely solved the problem for operators

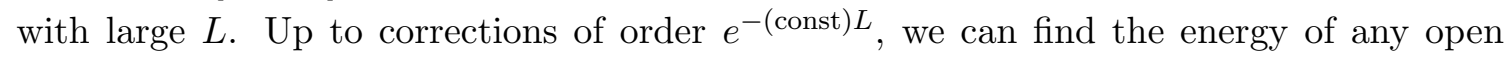
string state by solving the appropriate Asymptotic Bethe equations.

After we have found the boundary reflection matrix we can then consider the possibility of rotating the half Wilson line that is associated with it. This rotation will simply act on the indices of the reflection matrix via a global transformation. Now we can consider states of the form $B_{l} Z^{L} B_{r}(\theta, \phi)$, where we have rotated one of the sides of the Wilson line. This 


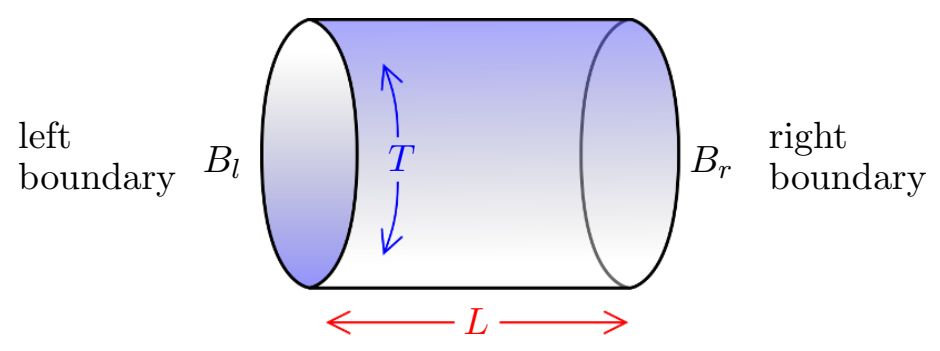

Figure 2. The BTBA trick. The same partition function can be viewed in two ways (1.7). In the open string channel it is a trace over all states in the open string Hilbert space. In this case Euclidean time runs along the $T$ arrow. Alternatively we can view it as the propagation of a closed string along the $L$ arrow. The closed string has length $T$ and propagates over a Euclidean time $L$. The two boundary conditions, now lead to two boundary states that create the closed strings that propagate along the closed string channel.

operator is no longer BPS but its energy is very small when $L$ is very large, i.e. it has zero energy up to $e^{- \text {(const)L }}$ corrections. These are called Luscher (or wrapping) corrections.

Before writing down the Thermodynamic Bethe Ansatz that describes the most general finite $L$ state, we will make some checks on the phase that has been obtained. As a nontrivial check one can get the first corrections to the ground state energy for large $L$. Namely, we are interested in the anomalous dimension of the operator of the form $B_{l} Z^{L} B_{r}(\theta, \phi)$. This correction is given by a Luscher-type formula. This formula can be most simply understood by considering the problem in the mirror picture. Namely, we exchange space and time in the open string picture. In other words, we have the equivalence

$$
Z_{B_{l}, B_{r}}^{\text {open }}=\operatorname{Tr}_{\text {open }}\left[e^{-T H_{B_{l}, B_{r}}^{\text {open }}}\right]=\left\langle B_{l}\left|e^{-L H_{\text {closed }}}\right| B_{r}\right\rangle,
$$

where $H_{B_{l}, B_{r}}^{\text {open }}$ is the open chain Hamiltonian on a strip of length $L$ and $H_{\text {closed }}$ is the closed chain Hamiltonian of the mirror theory on a circle of size $T$. So now we have a closed string exchanged between two boundary states. The analytic continuation of the boundary reflection matrix gives us the probability of emitting a pair of particles from the boundary state. It turns out that this continued reflection matrix has a pole at zero mirror momentum which implies that we can create single particles [17]. The coefficient of the pole in the reflection matrix at zero mirror momentum determines the prefactor of the Luscher correction [23]. We compute this at strong coupling and we find agreement with a direct string theory computation. Furthermore, the leading order correction at weak coupling, going like $g^{2}$, also comes from this Luscher type term. In this way we match the leading corrections at weak and strong coupling. This constitutes a test of the boundary reflection matrix. In particular the very existence of the pole at zero mirror momentum is due to the phase factor of the matrix, which we derived by solving the crossing equation.

Finally, one can write down a Thermodynamic Bethe Ansatz equation that describes the finite $L$ situation. This follows the standard route for getting the energies of states of an integrable field theory with a boundary. The derivation of these equations is very similar to the derivation of the equations for closed string states. The new element is that instead of a thermodynamic partition function we have the overlap between two boundary states, 
as in (1.7). The derivation of TBA equations for integrable systems with a boundary was considered in [18]. The boundary states are given in terms of the analytic continuation of the boundary reflection matrix. The TBA system of equations arises from evaluating this exact overlap between the two boundary states in an approximate way by giving the densities. Most of the TBA equations come from the entropy terms, which are the same in our case. Thus the boundary TBA equations are very similar in structure to the closed ones. We obtain

$$
\log Y_{A}=\log \left(\kappa_{A}^{l} \kappa_{A}^{r}\right)-2 L E_{m, A}+K_{A B} * \log \left(1+Y_{B}\right) .
$$

The cusp anomalous dimension, or quark/anti-quark potential is given schematically by

$$
\mathcal{E}=-\frac{1}{2 \pi} \sum_{A} \int_{0}^{\infty} d q_{A} \log \left(1+Y_{A}\right)
$$

Here $E_{m, A}$ and $q_{A}$ are the energies and momenta of the excitations in the mirror theory. The equations will be given below in their full detail, (4.2)-(4.6). The information about the boundary is contained in $\kappa_{A}$ which comes from the reflection phase of the theory and depends on the boundary state.

Note. We were informed that similar ideas were pursued in [24].

\section{Spectrum of operators on a Wilson line}

Let us first discuss the symmetries preserved by a straight Wilson line. Let us start with the bosonic symmetries. It preserves an $S L(2) \times S U(2) \times S O(5)$ symmetry group. The $S O(5)$ is the subset of $S O(6)$ that leaves $\Phi^{4}$ invariant, where $\Phi^{4}$ is the scalar that couples to the Wilson line. The $S U(2)$ factor corresponds to the spatial rotations around the loop. The $S L(2)$ factor contains time translations, dilatations and special conformal transformations along the time direction. In addition, we preserve half of the supercharges. The full supergroup is $O S p\left(4^{*} \mid 4\right)$. The star means it is the real form of $S O(4)$ such that $S O\left(4^{*}\right) \sim S L(2) \times S U(2)$.

Now we can consider the insertion of an operator of the form $Z^{L}$ on the Wilson loop, we can denote this as $B_{l} Z^{L} B_{r}$. Here we choose $Z$ to be $Z=\Phi^{5}+i \Phi^{6}$.

The operator $Z$ inserted at the origin preserves an $S U(2 \mid 2)^{2}$ subgroup of the full symmetry group of the theory. The Wilson loop, together with the $Z$ insertions at the origin preserve an $S U(2 \mid 2)_{D}$ subgroup of all the symmetry groups we mentioned. This is a diagonal combination of the two $S U(2 \mid 2)$ factors preserved by $Z$. This common preserved symmetry is very useful for analyzing this problem. These operators are BPS, and they have protected anomalous dimension, $\mathcal{E} \equiv \Delta-J_{56}=0$.

Note that on $S^{3}$ we have a flux tube that goes between the quark and the anti-quark. These operators inserted on the Wilson loop are mapped to to various excitations of the flux tube. 


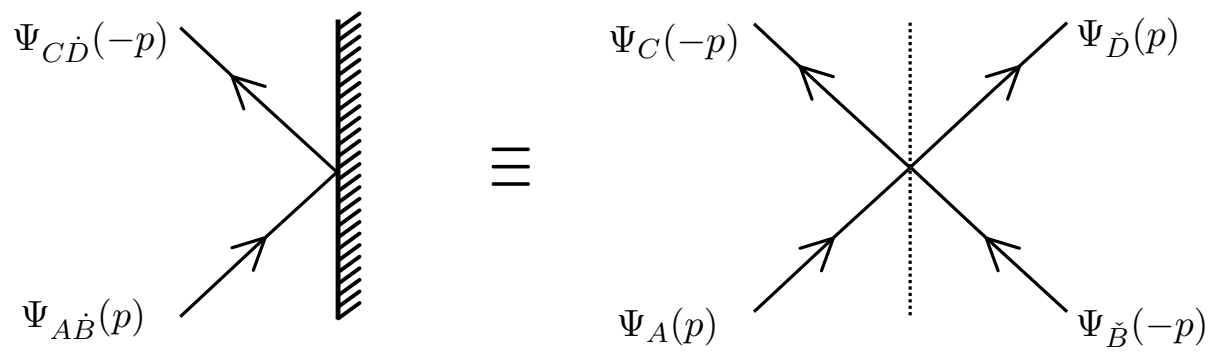

Figure 3. Unfolding of $R(p)$ into $S(p,-p)$. There is a non-trivial map between dotted and checked indices. See appendix A for details.

\subsection{The boundary reflection matrix}

Recall that the bulk excitations are in a fundamental representation of each of the two $\widetilde{s u}(2 \mid 2)$ factors of the $\widetilde{s u}(2 \mid 2)^{2}$ symmetry of the $Z$-vacuum. The tilde means that we are considering the momentum dependent central extensions discussed in [13, 25]. In other words, we can think of them as particles with two indices $\Psi_{A, \dot{B}}$, where $A$ labels the fundamental of the first $\widetilde{s u}(2 \mid 2)$ and $\dot{B}$ labels the fundamental of the second $\widetilde{s u}(2 \mid 2)$ factor of the $\widetilde{s u}(2 \mid 2)^{2}$ symmetry of the infinite chain. This central extension determines the dispersion relation for the excitations

$$
\begin{aligned}
\frac{i}{g} & =x^{+}+\frac{1}{x^{+}}-x^{-}-\frac{1}{x^{-}} \\
e^{i p} & =\frac{x^{+}}{x^{-}}, \quad \epsilon=i g\left(\frac{1}{x^{+}}-\frac{1}{x^{-}}-x^{+}+x^{-}\right)=\sqrt{1+16 g^{2} \sin ^{2} \frac{p}{2}},
\end{aligned}
$$

Throughout this paper we define $g$ as $^{2}$

$$
g \equiv \frac{\sqrt{\lambda}}{4 \pi}=\frac{\sqrt{g_{Y M}^{2} N}}{4 \pi} .
$$

The scattering matrix between two particles has the form $S_{A \dot{A}, B \dot{B}}^{C \dot{C} D \dot{D}}=S_{0}^{2} \hat{S}_{A B}^{C D} \hat{S}_{\dot{A} \dot{B}}^{\dot{C}}$ [13]. Namely, it is the product of a phase factor $S_{0}^{2}$ and two identical matrices, one for each $\widetilde{s u}(2 \mid 2)$ factor. These matrices are fixed (up to an overall factor) by the $\widetilde{s u}(2 \mid 2)$ symmetry of the theory $[13,25]$. These matrices depend on the two momenta, $p_{1}$ and $p_{2}$, of the scattered variables. The phase factor $S_{0}\left(p_{1}, p_{2}\right)$ was guessed in [6, 26], and a nice derivation was given in [21, 22].

In our problem we need to fix a reflection matrix of the form $R_{A \dot{B}}^{C \dot{D}}(p)$. Let us consider first the reflection from the right boundary, see figure 3 . This matrix depends on only one momentum $p$, the momentum of the incident magnon. The boundary is invariant under an $\widetilde{s u}(2 \mid 2)_{\mathrm{D}}$ symmetry group, which is diagonally embedded in the $\widetilde{s u}(2 \mid 2)^{2}$ symmetry group of the bulk of the spin chain (see appendix A). A similar problem was studied in [16] and the matrix part of the reflection is the same. Thus the symmetries constraining the

\footnotetext{
${ }^{2}$ Note that $g \neq g_{Y M}$.
} 
reflection matrix are exactly the same as those constraining the bulk scattering matrix for each of the $\widetilde{s u}(2 \mid 2)$ factors. From this argument we expect that the matrix structure should be completely fixed. In fact, the matrix structure should be essentially the same as what we encounter in the matrix $\hat{S}_{A B}^{C D}(p,-p)$, or $R_{A \dot{A}}^{C \dot{C}}(p) \propto \hat{S}_{A \dot{A}}^{C \dot{C}}(p,-p)$. One is tempted to say that the scattering phase factor would be $S_{0}(p,-p)$. However, this is not fixed by the symmetries, and will not be true as we discuss below. In the presence of a boundary, we can do a kind of "unfolding" of the spin chain. Here each bulk magnon is viewed as a pair of magnons of $\widetilde{s u}(2 \mid 2)_{\mathrm{D}}$, one with momentum $p$ to the left of the boundary and one with momentum $-p$ to the right of the boundary. See figure 3 .

This completely solves the problem of fixing the matrix structure of the reflection matrix. The full reflection matrix, in complete detail, is given in appendix A. One can also check that it obeys the boundary Yang Baxter equation. But this is clear from the "unfolded" picture in terms of a single chain. We should emphasize that we have assumed that there are no boundary degrees of freedom. We do not see any evidence of any boundary degrees of freedom at either weak or strong coupling, so this is a reasonable assumption.

Before we determine the phase, let us make a side remark. There is a variety of problems that give rise to a spin chain with boundaries and preserve the same symmetries, $O S p\left(4^{*} \mid 4\right)$. We can consider an open string ending on a D5 brane that wraps $A d S_{4} \times S^{2}$, or $A d S_{2} \times S^{4}$. In fact, there is a whole family of BPS branes of this kind that arises by adding flux for the $U(1)$ gauge field on the brane worldvolume on the $S^{2}$ or $A d S_{2}$. In fact, in the limit of large electric flux on the $A d S_{2} \times S^{4}$ brane we get a boundary condition like the Wilson loop one. In fact the $A d S_{2} \times S^{4}$ branes can be interpreted as Wilson loops in the $k$-fold antisymmetric representation of $U(N)$ [27]. In all these cases one can choose the BMN vacuum (or choose the field $Z$ ) in such a way that we preserve the $\widetilde{s u}(2 \mid 2)_{\mathrm{D}}$ of the spin chain. Therefore, we would get the same matrix structure for the reflection matrix, again assuming that there are no boundary degrees of freedom. However, they would differ in the choice of a phase factor. Below we get a phase factor which has all the right properties to correspond to the one of the Wilson loop. It would be interesting to fix the phase factor also for these other cases, but we leave this to the future.

In order to fix the phase factor we write a crossing equation. We derive this by writing the identity state of [13], scattering it through the boundary and demanding that the full phase is equal to one. Denoting the phase factor as $R_{0}$, defined more precisely in appendix A, we obtain the crossing equation

$$
R_{0}(p) R_{0}(\bar{p})=\sigma(p,-\bar{p})^{2}
$$

where the bar indicates the action of the crossing transformation. Here $\sigma\left(p_{1}, p_{2}\right)$ is the bulk dressing phase, discussed in $[6,22]$. We are going to $\bar{p}$ along the the same contour in momentum space that we choose in the formulation of the bulk crossing equation.

In addition, we also should impose the unitarity condition

$$
R_{0}(p) R_{0}(-p)=1
$$


We now write the ansatz

$$
R_{0}(p)=\frac{1}{\sigma_{B}(p) \sigma(p,-p)}\left(\frac{1+\frac{1}{\left(x^{-}\right)^{2}}}{1+\frac{1}{\left(x^{+}\right)^{2}}}\right) .
$$

Here $\sigma$ is the bulk dressing phase. This would be our naive choice for a phase factor. The explicit factors of $x^{ \pm}$have been chosen only to simplify the final formula. We have an unknown factor $\sigma_{B}(p)$. Now (2.4) becomes

$$
\sigma_{B}(p) \sigma_{B}(\bar{p})=\frac{x^{-}+\frac{1}{x^{-}}}{x^{+}+\frac{1}{x^{+}}}
$$

We can now solve this equation using the method proposed in [21, 22]. We give the details in appendix B. We obtain

$$
\begin{aligned}
\sigma_{B} & =e^{i \chi\left(x^{+}\right)-i \chi\left(x^{-}\right)}, \\
i \chi(x) & =i \Phi(x)=\oint_{|z|=1} \frac{d z}{2 \pi i} \frac{1}{x-z} \log \left\{\frac{\sinh \left[2 \pi g\left(z+\frac{1}{z}\right)\right]}{2 \pi g\left(z+\frac{1}{z}\right)}\right\}, \quad|x|>1 .
\end{aligned}
$$

This expression is valid when $|x|>1$. The value for $\chi$ in other regions is given by analytic continuation. We have also introduced the function $\Phi(x)$ which is given by the integral for all values of $x$. When $|x|<1$ these two functions differ by

$$
i \chi(x)=i \Phi(x)+\log \left\{\frac{\sinh \left[2 \pi g\left(x+\frac{1}{x}\right)\right]}{2 \pi g\left(x+\frac{1}{x}\right)}\right\}, \quad|x|<1
$$

The ambiguities in the choice of branch cuts for the logarithm cancel out when we compute $\sigma_{B}$ in (2.8). Note that $\chi(x)=\chi(-x)$.

So far, we have found a particular solution of the boundary crossing equation. Still, the true dressing phase might require the inclusion of further CDD factors. In order to make a conjecture for the exact boundary dressing phase, we need to compare against some explicit computations.

Before doing so, let us observe that, given $\sigma_{B}(p)$, we can define an infinite family of solutions by taking

$$
\sigma_{B}^{(s)}(p)=\left(\frac{x^{-}+\frac{1}{x^{-}}}{x^{+}+\frac{1}{x^{+}}}\right)^{s}\left[\sigma_{B}(p)\right]^{1-2 s} .
$$

By computing the dressing phase in the physical regime we will be able to show that $s=0$ is the solution we want.

The proposal for the phase factor for the reflection matrix, given in (2.6), (2.8) is one of the important results of this paper. We will perform various checks on its validity.

\subsection{Checks of the boundary reflection phase in the physical region}

Let us describe how to compute the boundary dressing phase at strong coupling. We have to consider the open string solution that corresponds to a $1 / 2$ BPS Wilson line carrying 


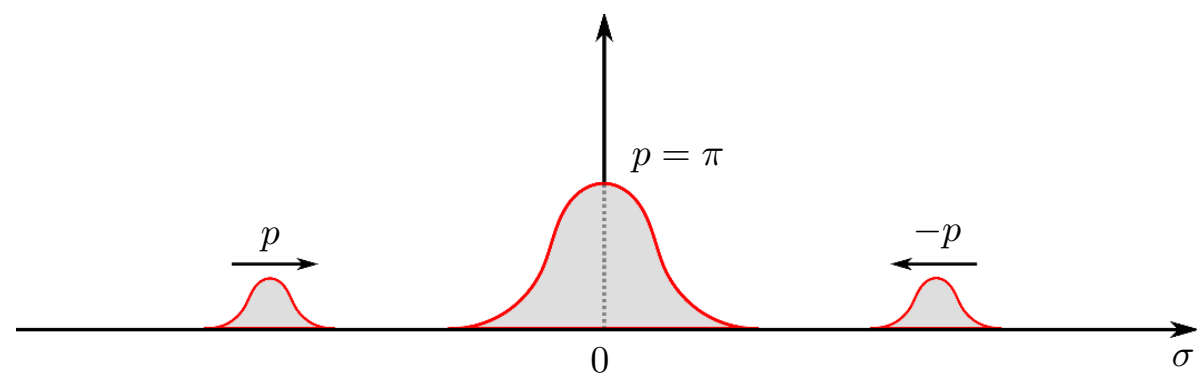

Figure 4. Computation of the reflection phase at strong coupling. We have a soliton at the boundary, which is at rest at $\sigma=0$. There is also an image soliton coming from the right. Then the soliton with momentum $p$ scatters through the soliton at rest and the one with momentum $-p$, leading to a certain time delay. From the time delay we can compute the derivative of the reflection phase with respect to the energy.

a large $J_{56}$ charge given in [11]. This solution describes the transition from the boundary Wilson line to an infinite BMN vacuum. It is convenient to understand this solution in the conformal gauge, when we set the stress tensor on the $S^{5}$ equal to one, and the stress tensor of the $A d S_{5}$ to minus one. In these variables, the problem only involves an $A d S_{2} \times S^{2}$ subspace and we can perform the Pohlmeyer reduction in each factor. The $S^{2}$ part gives rise to a sine gordon theory and the solution is just half of a soliton at rest. More precisely, the center of the sine gordon soliton sits at the boundary. In the $A d S_{2}$ part we have a sinh gordon theory, and the solution is a sinh-gordon "soliton". This is a singular solution which is the direct analytic continuation of the sine gordon soliton. The singularity reflects the fact that the string goes to the AdS boundary. If we compute the energy, there is a divergent part and the finite part is zero. The setup is explained in more detail in [11]. The fact that the finite part of the energy is zero is consistent with the absence of a boundary impurity transforming non-trivially under $\widetilde{s u}(2 \mid 2)$. A bulk magnon is a sine gordon soliton, and leaves the AdS part of the solution unperturbed. In the presence of a boundary, we need to put also the "image" of this soliton and the configuration looks as in figure 4. The reflection involves the scattering of the soliton with the image soliton as well as the scattering with the soliton at rest. These soliton scattering phases were computed in $[14,28]$. So the strong coupling limit of the right boundary scattering phase $R_{0}(p)=e^{i \delta_{\mathrm{R}}(p)}$ is given by

$$
\delta_{\mathrm{R}}(p)=-8 g \cos \frac{p}{2} \log \cos \frac{p}{2}-4 g \cos \frac{p}{2} \log \left(\frac{1-\sin \frac{p}{2}}{1+\sin \frac{p}{2}}\right)
$$

The first term in (2.12) is exactly what one gets from the strong coupling limit of the factor $1 / \sigma(p,-p)[28]$. We will see that the second term corresponds to $\sigma_{B}(p)^{-1}$. At strong coupling can expand (2.9) as

$$
i \chi(x) \sim 4 g\left[-1+\left(x+\frac{1}{x}\right) \frac{1}{2 i} \log \frac{(x+i)}{(x-i)}\right]+\mathcal{O}(1),
$$


which leads, for physical excitations, to

$$
\frac{1}{i} \log \sigma_{B}(p)=4 g \cos \frac{p}{2} \log \left(\frac{1-\sin \frac{p}{2}}{1+\sin \frac{p}{2}}\right) .
$$

This indicates that we must pick the case $s=0$ from the family of solutions (2.11).

Finally, let us discuss the behavior at weak coupling. The bulk dressing phase $\sigma$ has its first contribution at order $g^{6}$, leading to four loop corrections to anomalous dimensions. On the other hand, the boundary dressing phase, $\sigma_{B}$, receives its first contribution at $g^{4}$, so that it will start modifying anomalous dimensions of operators inserted on the Wilson loop (dual to open string states) at three loops.

\subsection{Reflection matrix for a Wilson line at general angles}

We will need the boundary reflection matrix for a Wilson line sitting at general angles, $\phi$ and $\theta$. In particular, we want the left and right boundaries of the open chain to be rotated by relative angles. We can obtain the boundary state of the Wilson line at a different position on the $S^{3}$, or the $S^{5}$, by applying a symmetry transformation on $B_{r}$. This should be a symmetry that is broken by $B_{r}$. So for example, we can apply an $S U(2)_{L}$ rotation on the $S^{3}$ which is in one of the $S U(2)$ factors in the $S O(4)$ group of rotations of the 3 -sphere. If we apply an $S U(2)_{L}$ rotation with an angle $2 \phi$, we will get that the quark is rotated by an angle $\phi$ on the $S^{3}$, away from the south pole. See figure 1(b). Note that the $S U(2)_{L}$ we are considering is a symmetry of the $Z$ vacuum. We have a similar feature on the $S^{5}$. We can also apply a rotation in an $S U(2)_{L^{\prime}}$ factor inside $S O(4) \subset S O(6)$ (this $S O(4)$ leaves the $Z$ vacuum invariant). The reflection matrix is very easy to obtain. We pick these two $S U(2)$ generators so that they sit in the bosonic part of one of the $\widetilde{s u}(2 \mid 2)$ factors of the $\widetilde{s u}(2 \mid 2)^{2}$ symmetry of the bulk. Then they will simply introduce some phases of the form $e^{i \phi}$ or $e^{i \theta}$ when a state is reflected from the boundary and its $S U(2)$ quantum number changes. The $S U(2)_{L}$ or $S U(2)_{L^{\prime}}$ quantum numbers can change because they are not symmetries of the boundary state. More explicitly, the reflection matrix from a boundary state at angles $\phi, \theta$ is given by

$$
R_{A \dot{A}}^{B \dot{B}}(\theta, \phi)=\left(m^{-1}\right){ }_{D}^{B} m_{A}^{C} R_{C \dot{A}}^{D \dot{B}}(0,0), \quad \text { with } \quad m=\operatorname{diag}\left(e^{i \theta}, e^{-i \theta}, e^{i \phi}, e^{-i \phi}\right) .
$$

Note that the matrix $m$ acts only on the undotted indices since we did a rotation inside only one of the $\widetilde{s u}(2 \mid 2)$ factors.

\subsection{Luscher computations and checks in the mirror region}

In this subsection we start considering the problem with two boundaries. In other words the operator $B_{l} Z^{L} B_{r}(\phi, \theta)$. Here $\phi, \theta$ are the relative orientations of the two boundaries. On the plane, this corresponds to a cusp, plus an operator of the form $Z^{L}$ at the tip. In the limit $L \gg 1$ we get the naive superposition of the two boundaries and the energy of the state is zero $(\mathcal{E}=\Delta-L=0)$, regardless of the orientation of the two boundaries. The leading correction is of the form $e^{- \text {(constant) } L}$. These corrections come from the exchange of particles along the "mirror" channel. The boundary sources particles, which then travel to 
the other boundary. These corrections sometimes go under the name of Luscher corrections. Of course the familiar Yukawa potential is a simple example where the leading correction comes from the exchange of a single massive particle.

In order to derive the precise correction formula it is convenient to describe in more detail the mirror theory. In the bulk of the worldsheet the mirror theory was discussed in various papers, see [29] for example. This theory is defined by exchanging the space and time directions of the spin chain we have been considering so far. Thus, instead of (2.2) we define $q=i \epsilon$ and $E_{m}=i p$, and use the same formulas as in (2.2). Here $q$ is the mirror momentum and $E_{m}$ is the mirror energy. In order for these to be real we will need to pick a solution of (2.1) with $\left|x^{+}\right|>1$ and $\left|x^{-}\right|<1$. From the expression for $q$, we can write

$$
\begin{aligned}
z^{[ \pm a]} & =\frac{1}{4 g}\left(\sqrt{1+\frac{16 g^{2}}{a^{2}+q^{2}}} \pm 1\right)(q+i a), \\
E_{m} & =2 \operatorname{arcsinh} \frac{\sqrt{q^{2}+a^{2}}}{4 g}
\end{aligned}
$$

Here $z^{ \pm}$just denote the values of $x^{ \pm}$in the mirror region. We have also written the dispersion relation in the mirror region, for an arbitrary bound state. The elementary mirror magnon has $a=1$.

When we have a boundary, this time/space flip turns the boundary into a boundary state, see figure 2 . Then a suitable analytic continuation of the boundary reflection matrix characterizes the boundary state. The boundary state creates a supersposition of many particles. The total mirror momentum should be zero since it is translational invariant. So, schematically the state has the form

$$
|B\rangle=|0\rangle+\int_{0}^{\infty} \frac{d q}{2 \pi} K^{A \dot{A}, B \dot{B}}(q) a_{-q A \dot{A}}^{\dagger} a_{q B \dot{B}}^{\dagger}|0\rangle+\cdots
$$

with

$$
K^{A \dot{A}, B \dot{B}}(q)=\left[R^{-1}\left(z^{+}, z^{-}\right)\right]_{D \dot{D}}^{A \dot{A}} \mathcal{C}^{D \dot{D}, B \dot{B}}
$$

where we put the mirror values (2.16). Here $R$ is the right reflection matrix, with $z^{ \pm}$ continued to the mirror region (2.16). This amounts to an analytic continuation of the reflection matrix. Here $a_{q A \dot{A}}^{\dagger}$ is the creation operator of a magnon with momentum q. $\mathcal{C}$ is a charge conjugation matrix. In the case of a relativistic model with a single particle (2.19) reads $K(\theta)=1 / R\left(\theta-i \frac{\pi}{2}\right)$, [17]. The formula (2.19) can be obtained by performing a $\pi / 2$ rotation of the boundary condition. Due to the independence of reflection events from a boundary, we can exponentiate (2.19) to get the full boundary state [17, 18]. Similarly, we can form a future boundary state. This is a boundary state that annihilates the particles. It is given by

$$
\langle B|=\langle 0|+\langle 0| \int_{0}^{\infty} \frac{d q}{2 \pi} \bar{K}_{A \dot{A}, B \dot{B}}(q) a_{q}^{A \dot{A}} a_{-q}^{B \dot{B}}+\cdots
$$


with

$$
\bar{K}_{A \dot{A}, B \dot{B}}(q)=\left[R^{-1}\left(-\frac{1}{z^{-}},-\frac{1}{z^{+}}\right)\right]_{B \dot{B}}^{D \dot{D}} \mathcal{C}_{D \dot{D} A \dot{A}}
$$

In the relativist case $(2.21)$ would be $\bar{K}(\theta)=\frac{1}{R\left(-i \frac{\pi}{2}-\theta\right)}$.

When $L$ is very large the leading $L$-dependent contribution comes from the exchange of this pair of particles and we can write the corresponding contribution as

$$
\delta \mathcal{E}=-\int_{0}^{\infty} \frac{d q}{2 \pi} e^{-2 L E_{m}(q)} t(q), \quad t(q)=\operatorname{Tr}[K(q) \bar{K}(q)] .
$$

This formula is correct whenever the integral is finite.

In our case, the phase factor $\sigma_{B}$ has a pole at $q=0$. In the physical region $\sigma_{B}(p)$ was perfectly finite. This pole in the mirror region is crucial for obtaining the correct answer. But first we need to generalize (2.22) to the situation when we have a pole at $q=0$. The physical interpretation of this pole at $q=0$ is that the boundary state is sourcing single particles states in the mirror theory [17]. For a similar case in the AdS/CFT context see [30]. Obviously such source has to contain only zero momentum particles.

A careful analysis leads to the formula [23]

$$
\mathcal{E} \sim-\int_{0}^{\infty} \frac{d q}{2 \pi} \log \left\{1+e^{-2 L E_{m}(q)} \operatorname{Tr}[K(q) \bar{K}(q)]\right\} \sim-\frac{1}{2} e^{-L E_{m}(0)} \sqrt{\left.q^{2} \operatorname{Tr}[K(q) \bar{K}(q)]\right|_{q=0}} .
$$

In the last equality we extracted the leading term in the integral, which comes only from the coefficient of the pole. Notice that the $L$ dependence is precisely what we expect from the exchange of a single particle. We should sum over all the particles that can be exchanged. The mirror theory contains bound states indexed by an integer $a=1,2, \cdots$, and we should sum over them.

In appendix $\mathrm{D}$ we show that we can evaluate $t(q)$ for a fundamental mirror particle and we obtain

$$
t(q)=\sigma_{B}\left(z^{+}, z^{-}\right) \sigma_{B}\left(-\frac{1}{z^{-}},-\frac{1}{z^{+}}\right)\left(\frac{z^{-}}{z^{+}}\right)^{2}\left(\operatorname{Tr}\left[(-1)^{F}\right]\right)^{2},
$$

where the trace is over the four states of a single $\widetilde{s u}(2 \mid 2)$ magnon. Let us now give a simple explanation for this formula, for more details see appendix D. We can write the reflection matrices that appear in $K$ and $\bar{K}(2.19)$ (2.21) in terms of bulk S-matrices for the unfolded theory, namely in terms of bulk $S$ matrices for a single $\widetilde{s u}(2 \mid 2)$ factor. The matrix in $K$ is essentially $\mathcal{S}(-p, p)$ and the one in $\bar{K}$ is $\mathcal{S}(\bar{p},-\bar{p})$. When we multiply these matrices we can use the bulk crossing equation to get the identity. Here we should use the full bulk matrix, including the bulk $\sigma$ factor. This is the reason that the bulk $\sigma$ factor disappears from the final formula (2.24), but the boundary one remains. The factor of $z^{-} / z^{+}$arises from the factor in parenthesis in (2.6). Finally, the $(-1)^{F}$ is related to the fact that we have fermions. Here $F$ is the fermion number. When we perform the TBA trick, we get periodic fermions in Euclidean time if we started with periodic fermions in the spatial directions. Of 


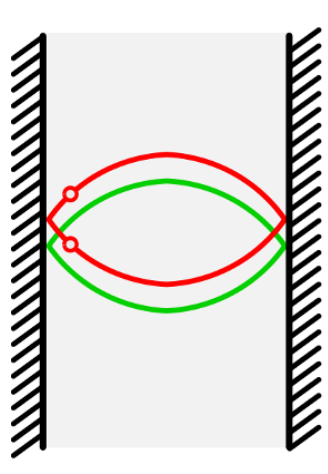

(a)

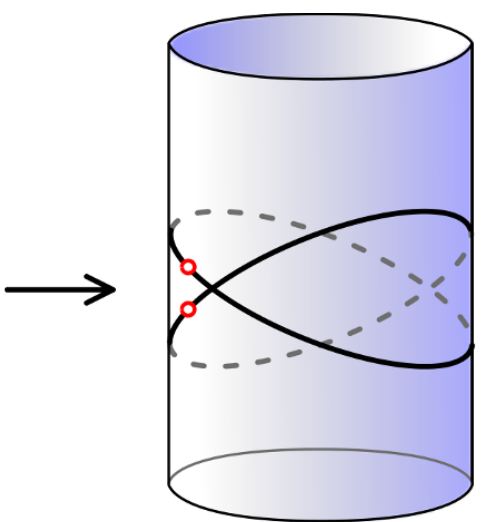

(b)

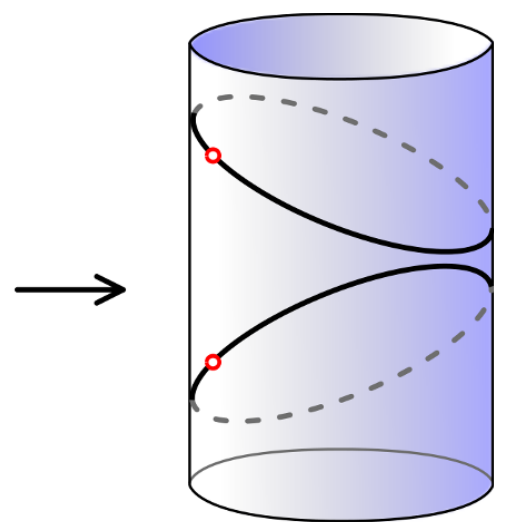

(c)

Figure 5. (a) We have a strip with pairs of particles being exchanged. The two colors represent the two types of indices. In (b) we unfolded this into a cylinder computation. The $K$ matrices became $S$ matrices for a single $\widetilde{s u}(2 \mid 2)$. (c) Using crossing we have moved the lines. The red circles indicates the action of the matrix $m$.

course a periodic fermion in Euclidean time is the same as the trace with a $(-1)^{F}$ inserted. The operations that lead to $(2.24)$ can be understood graphically as in figure 5 .

Of course, for a fundamental magnon $\operatorname{Tr}\left[(-1)^{F}\right]=0$. This is good, since it is saying that the correction vanishes in the BPS situation. If we rotate one boundary relative to the other then we need to perform the replacement

$$
\operatorname{Tr}\left[(-1)^{F}\right] \longrightarrow \operatorname{Tr}\left[(-1)^{F} m\right]=-2(\cos \phi-\cos \theta) .
$$

where $m$ is given in (2.15). Again, we see that it vanishes in the BPS case $\phi= \pm \theta$.

To write down the full Luscher formula we need to compute $t(q)$ also for the bound states of the mirror theory. One can first use the standard fusion procedure to get the bound state reflection matrix. Then one can use the same argument as above to eliminate the bulk $S$ matrices, as in figure 5 . The final formula is

$$
\begin{gathered}
t_{a}(q)=\sigma_{B}\left(z^{[+a]}, z^{[-a]}\right) \sigma_{B}\left(-\frac{1}{z^{[-a]}},-\frac{1}{z^{[+a]}}\right)\left(\frac{z^{[-a]}}{z^{[+a]}}\right)^{2}\left(\operatorname{Tr}\left[(-1)^{F} m_{a}\right]\right)^{2} \\
\operatorname{Tr}\left[(-1)^{F} m_{a}\right]=(-1)^{a} 2(\cos \phi-\cos \theta) \frac{\sin a \phi}{\sin \phi}
\end{gathered}
$$

where now the trace is over all the states of a magnon boundstate in a single copy of $\widetilde{s u}(2 \mid 2)$, see equation (D.7) in appendix D. As anticipated, an important property of $\sigma_{B}$ is that it has a pole at $q=0$. More precisely the combination of $\sigma_{B}$ in (2.26) becomes

$$
\begin{aligned}
e^{i \chi\left(z^{[+a]}\right)-i \chi\left(z^{[-a]}\right)+i \chi\left(1 / z^{[-a]}\right)-i \chi\left(1 / z^{[+a]}\right)}= & \frac{2 \pi g\left(z^{[-a]}+\frac{1}{z^{[-a]}}\right)}{\sinh \left[2 \pi g\left(z^{[-a]}+\frac{1}{z^{[-a]}}\right)\right]} \frac{2 \pi g\left(z^{[+a]}+\frac{1}{z^{[+a]}}\right)}{\sinh \left[2 \pi g\left(z^{[+a]}+\frac{1}{z^{[+a]}}\right)\right]} \\
& \times e^{i\left(\Phi\left(z^{[+a]}\right)-\Phi\left(z^{[-a]}\right)+\Phi\left(1 / z^{[-a]}\right)-\Phi\left(1 / z^{[+a]}\right)\right)} \cdot
\end{aligned}
$$


Here we used that $z$ is in the mirror kinematics and we used (2.10) to evaluate $\chi(x)$ when $|x|<1$. We have also used that $\chi(-x)=\chi(x)$. Each of the sinh factors leads to a pole at $q=0$. Namely, using (2.16) we get

$$
2 \pi g\left(z^{[ \pm a]}+\frac{1}{z^{[ \pm a]}}\right)= \pm i \pi a+\pi q \sqrt{1+\frac{16 g^{2}}{a^{2}}}+\mathcal{O}\left(q^{3}\right) .
$$

for small $q$. We then can write the pole part of (2.28) as

$$
\begin{aligned}
e^{i \chi\left(z^{[+a]}\right)-i \chi\left(z^{[-a]}\right)+i \chi\left(1 / z^{[-a]}\right)-i \chi\left(1 / z^{[+a]}\right)} & \sim \frac{1}{q^{2}} \frac{a^{4}}{\left(a^{2}+16 g^{2}\right)} F(a, g)^{2}+\mathcal{O}(1) \\
\text { with } \quad F(a, g)^{2} & \left.\equiv e^{i\left(\Phi\left(z^{[+a]}\right)-\Phi\left(z^{[-a]}\right)+\Phi\left(1 / z^{[-a]}\right)-\Phi\left(1 / z^{[+a]}\right)\right)}\right|_{q=6}
\end{aligned}
$$

where the last factor is evaluated at $q=0$.

Then we find the coefficient of the double pole of $t$ as

$$
\lim _{q \rightarrow 0} q^{2} t_{a}(q)=4 \frac{(\cos \phi-\cos \theta)^{2}}{\sin ^{2} \phi} \sin ^{2}(a \phi) \frac{a^{4}}{\left(a^{2}+16 g^{2}\right)}\left(\frac{-a+\sqrt{a^{2}+16 g^{2}}}{a+\sqrt{a^{2}+16 g^{2}}}\right)^{2} F(a, g)^{2} .
$$

The factor in parenthesis is $\left(z^{[-a]} / z^{[a]}\right)^{2}$. Finally, inserting this into the expression for the energy (2.23), we find

$$
\Delta \mathcal{E} \sim-\frac{(\cos \phi-\cos \theta)}{\sin \phi} \sum_{a=1}^{\infty}(-1)^{a}\left(\frac{-1+\sqrt{1+16 g^{2} / a^{2}}}{1+\sqrt{1+16 g^{2} / a^{2}}}\right)^{1+L} \sin (a \phi) \frac{a}{\sqrt{1+16 g^{2} / a^{2}}} F(a, g)
$$

The factor in parenthesis is just $e^{-E_{m}(a)(L+1)}$, representing the exchange of a bound state in the mirror channel. The sign $(-1)^{a}$ is a bit subtle and has to do with the correct sign we should pick for the square root in (2.23). The correct sign is easier to understand for an angle of the form $\phi=\pi-\delta$, for small $\delta$. In this case we have a quark antiquark configuration and it is clear that we should get a negative contribution to the energy. In fact, we can think of the overlap of the two boundary states as computing a kind of norm or inner product. We see that in terms of $\delta$ the expression has the expected sign. In other words, for small $\delta$ we get the positive sign of the square root in (2.23). Of course, once we get the expression for small $\delta$ we can write it in terms of $\phi$, or even analytically continue $\phi=-i \varphi$.

\subsubsection{Leading Luscher correction at weak coupling}

The expression (2.33) gives the leading Luscher correction at all values of the coupling for large $L$. Let us now examine it at weak coupling. Then the factor in parenthesis in (2.33) is of order $g^{2}$. So, at leading order, we get a term of the form $g^{2+2 L}$. This has the interesting implication that this leading "wrapping" correction appears at $L+1$ loops. In particular for $L=0$, the one loop contribution comes from such a term!. In fact, expanding (2.33) to 
leading order in $g^{2}$, and setting $L=0$, we can set $F=1$ to this order and obtain

$$
\begin{aligned}
\Gamma_{\text {cusp }} & =-4 g^{2} \frac{(\cos \phi-\cos \theta)}{\sin \phi} \sum_{a=1}^{\infty}(-1)^{a} \frac{\sin a \phi}{a} \\
& =2 g^{2} \frac{(\cos \phi-\cos \theta)}{\sin \phi} \phi,
\end{aligned}
$$

which coincides exactly with the leading 1-loop contribution to $\Gamma_{\text {cusp }}(\phi, \theta)$ computed in [2].

We can also do the computation of the leading order term for any $L$, we get

$$
\mathcal{E}=-g^{2+2 L} \frac{(\cos \phi-\cos \theta)}{\sin \phi} \frac{(-1)^{L}(4 \pi)^{1+2 L}}{(1+2 L) !} B_{1+2 L}\left(\frac{\pi-\phi}{2 \pi}\right)+\mathcal{O}\left(g^{4+2 L}\right)
$$

where $B_{n}(x)$ is the Bernoulli polynomial, which is a polynomial of degree $2 L+1$. In [5] a particular class of diagrams was identified which produced the same expression.

\subsubsection{Leading Luscher correction at strong coupling}

We can also compute the leading large $L$ correction at strong coupling. We simply evaluate the large $g$ limit of (2.33). First we note that

$$
\left(\frac{z^{[-a]}}{z^{[+a]}}\right)^{L+1} \sim e^{-\frac{a}{2 g} L}=e^{-L E_{m}(q=0)} .
$$

This implies that to leading order in $e^{-L}$ we only need to consider the case $a=1$. The expansion of the function $F$ is done in appendix C.1 eq. (C.31). Putting everything together we find that the leading strong coupling correction goes as

$$
\mathcal{E}=(\cos \phi-\cos \theta) \frac{16 g}{e^{2}} e^{-\frac{L}{2 g}}
$$

This agrees precisely with the result computed directly from classical string theory in appendix C, see (C.23). This constitutes a nontrivial check of the reflection phase. Notice, the funny factor of $e^{-2}$ which is correctly matched.

\section{The open Asymptotic Bethe Ansatz equations}

We will now write down the asymptotic Bethe ansatz (ABA) equations that describe the spectrum of operators with large $L$ inserted on the Wilson loop. These give rise to a spin chain with two boundaries, which are separated by a large distance $L$. Moreover, the ABA equations are used to derive the BTBA system by embedding them into the closed equations, as we do in appendix E.1.

In order to obtain the $\mathrm{ABA}$ equations we have to diagonalize the way the bulk and boundary scattering matrices act. This can be done by formulating a nested Bethe ansatz, which defines impurities at different levels of nesting. Here we just sketch the computation, which is a straightforward generalization of the case with periodic boundary conditions studied in [13]. 
Consider an asymptotic state with $N^{\mathrm{I}}$ bulk magnons, or level I excitations, on the half-line with a right boundary. We will introduce a second boundary and relative angles later, when writing down the Bethe equations. In particular, we can consider a state whose level I impurities all carry the same $S U(2 \mid 2)_{\mathrm{D}}$ index. ${ }^{3}$ Say, for example, in the unfolded notation,

$$
\left|\Psi_{3}\left(p_{1}\right) \cdots \Psi_{3}\left(p_{N^{\mathrm{I}}}\right) \Psi_{\check{3}}\left(-p_{N^{\mathrm{I}}}\right) \cdots \Psi_{\check{3}}\left(-p_{1}\right)\right\rangle \equiv|0\rangle^{\mathrm{II}},
$$

which is regarded as the level II vacuum state. Of course, we could also consider states where $N^{\mathrm{II}}$ out of the $N^{\mathrm{I}}$ level I impurities have different indices. Those should be understood as $N^{\mathrm{II}}$ impurities in the level II vacuum state. In total, such states will contain $N^{\mathrm{I}}$ level I impurities and $N^{\mathrm{II}}$ level II impurities. In general, we have $\left|\Psi_{a_{1}}\left(y_{1}\right) \cdots \Psi_{a_{N^{\mathrm{II}}}}\left(y_{N^{\mathrm{II}}}\right)\right\rangle^{\mathrm{II}}$ for $a_{k}=1,2$, where $y_{k}$ are auxiliary parameters associated with the level II impurities.

Similarly, a third level of nesting can be defined. If all the level II excitations carry the same index, for instance $\left|\Psi_{1}\left(y_{1}\right) \cdots \Psi_{1}\left(y_{N^{\mathrm{II}}}\right)\right\rangle^{\mathrm{II}} \equiv|0\rangle^{\mathrm{III}}$, we can define a level III vacuum

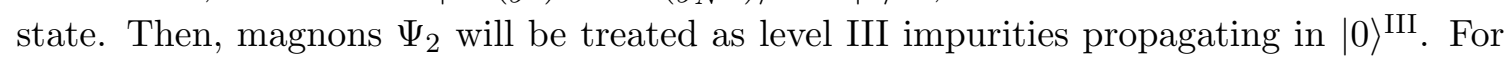
the kind of $S U(2 \mid 2)$ spin chain we are considering, this level III is the final level of nesting. ${ }^{4}$

Then, to formulate a coordinate Bethe ansatz, bulk and boundary scattering factors among excitations of different levels have to be introduced to write the nested wavefunctions. Those can be determined by imposing certain compatibility conditions. Namely, that the action of the bulk and boundary scattering matrices on wavefunctions with higher level impurities just pulls out the same factor as when acting on the level II vacuum state. Naturally, the bulk scattering factors are exactly the same as the ones obtained in the periodic case [13],

$$
\begin{aligned}
S^{\mathrm{I}, \mathrm{I}}\left(x_{1}^{ \pm}, x_{2}^{ \pm}\right) & =-S_{0}\left(p_{1}, p_{2}\right), \\
S^{\mathrm{I}, \mathrm{II}}\left(x^{ \pm}, y\right) & =1 / S^{\mathrm{II}, \mathrm{I}}\left(y, x^{ \pm}\right)=-\frac{y-x^{-}}{y-x^{+}}, \\
S^{\mathrm{III}, \mathrm{II}}(w, y) & =\frac{w-y-\frac{1}{y}+\frac{i}{2 g}}{w-y-\frac{1}{y}-\frac{i}{2 g}}=\frac{w-v+\frac{i}{2 g}}{w-v-\frac{i}{2 g}}, \\
S^{\mathrm{III}, \mathrm{III}}\left(w_{1}, w_{2}\right) & =\frac{w_{1}-w_{2}-\frac{i}{g}}{w_{1}-w_{2}+\frac{i}{g}},
\end{aligned}
$$

where

$$
S_{0}\left(p_{1}, p_{2}\right)^{2}=\frac{\left(x_{1}^{+}-x_{2}^{-}\right)\left(1-\frac{1}{x_{1}^{-} x_{2}^{+}}\right)}{\left(x_{1}^{-}-x_{2}^{+}\right)\left(1-\frac{1}{x_{1}^{+} x_{2}^{-}}\right)} \frac{1}{\sigma\left(p_{1}, p_{2}\right)^{2}}
$$

is the bulk dressing factor and $v=y+\frac{1}{y}$. All other bulk scattering factors are trivial.

The reflection factors can be derived in the same way. The level II vacuum (3.1) containing $N^{\mathrm{I}}$ magnons becomes a lattice with $2 N^{\mathrm{I}}$ sites. Consider a single level II impurity propagating in this vacuum from left, i.e. propagating along the left (undotted) indices of the bulk magnons. Undotted and dotted indices can only mix by the reflection of the

\footnotetext{
${ }^{3}$ The choice of index is arbitrary.

${ }^{4} \Psi_{4}$ are not considered as elementary but as double excitations.
} 


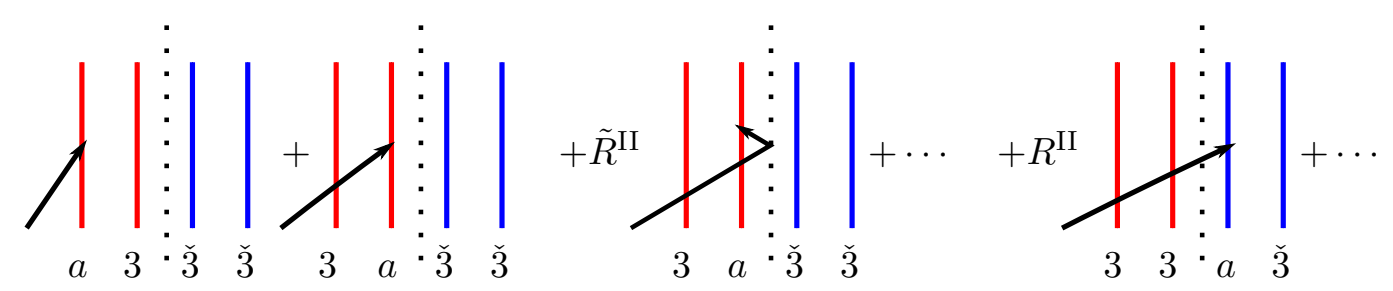

Figure 6. Propagation of a single level II impurity across the defect.

rightmost bulk magnon. That could make us think there exists a defect in the middle of the level II vacuum lattice which separates the 3 and $\check{3}$ indices of the rightmost level I impurity. In principle, the level II impurity could be reflected and transmitted across such defect, see figure 6.

However, and because the boundary scattering matrix $R(p) \propto S(p,-p)$, the compatibility condition we obtain from the reflection of the rightmost level I impurity is analogous to the ones we obtain from the scattering of two level I impurities. In this way, the compatibility conditions imply that level II impurities are purely transmitted. In other words $\tilde{R}^{\mathrm{II}}=0$ and $R^{\mathrm{II}}=1$. Analogously, the reflection of level III impurities is determined. In summary, we have

$$
R^{\mathrm{I}}\left(x^{ \pm}\right)=R_{0}(p), \quad R^{\mathrm{II}}(y)=1, \quad R^{\mathrm{III}}(w)=1,
$$

where $R_{0}(p)$ is the boundary phase factor (2.6).

Let us now put the system in a finite strip by introducing another boundary. We will then have certain quantization conditions on the rapidities for all kind of excitations, namely the Bethe ansatz equations. We will introduce the left boundary with relative angles with respect to the right one, by using the rotation discussed in section 2.3. To understand how this rotation affects the factors $R^{\mathrm{I}}, R^{\mathrm{II}}$ and $R^{\mathrm{III}}$ it is enough to consider the action of $m$, defined in (2.15), on the following key components of the reflection matrix

$$
\begin{aligned}
& R_{3 \check{3}}^{3 \check{3}} \mapsto R_{3 \check{3}}^{3 \check{3}}, \Rightarrow R^{\mathrm{I}} \mapsto R^{\mathrm{I}}, \\
& R_{1 \check{3}}^{3 \check{1}} \mapsto e^{i \theta-i \phi} R_{1 \check{3}}^{3 \check{1}}, \Rightarrow R^{\mathrm{II}} \mapsto e^{i \theta-i \phi} R^{\mathrm{II}}, \\
& R_{2 \check{1}}^{1 \check{2}} \mapsto e^{-2 i \theta} R_{2 \check{1}}^{1 \check{2}}, \Rightarrow R^{\mathrm{III}} \mapsto e^{-2 i \theta} R^{\mathrm{III}} \text {, }
\end{aligned}
$$

Let us finally write down the nested Bethe ansatz equations. They are obtained by picking an impurity of any level of nesting and moving it through all the other impurities twice and reflecting it from both boundaries as it is shown in the left picture of figure 7 .

If we go to the unfolded picture what we have is periodic chain of length $2 L$, where for every level I excitation of momentum $p_{k}$ there exists a mirrored one of momentum $-p_{k}$, figure 7. Such duplication does not occur for higher levels of nesting, for which the excitations do not necessarily come in pairs. When moving around the level I excitations to derive the Bethe equations, we have to recall that their duplication is an artifact of the unfolding. Every pair represents a single magnon in the original picture. When we move the original magnon, it looks like moving the pair simultaneously in the unfolded picture. Then, for level I impurities we pick up the factors that correspond to simultaneously moving 


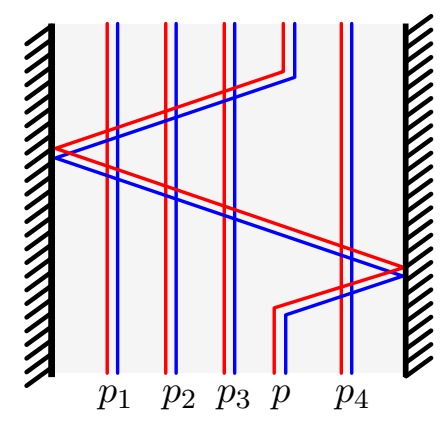

(a)

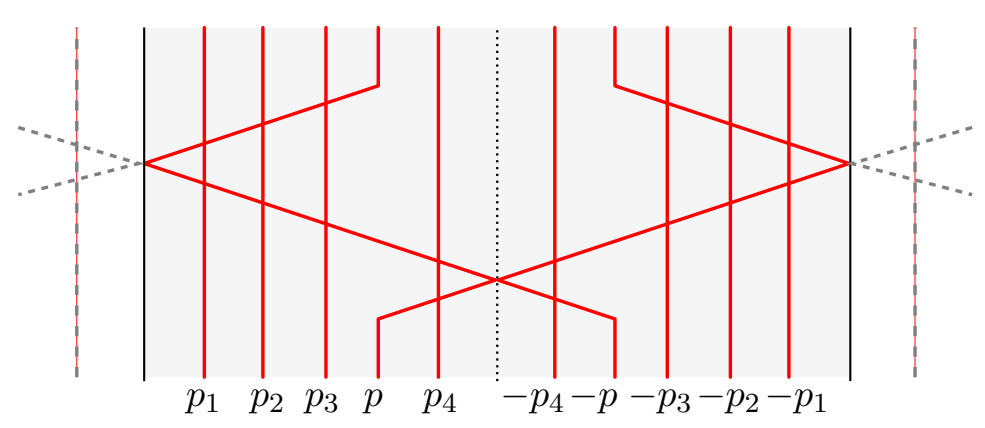

(b)

Figure 7. Bethe equation for the open chain. (a) The original picture with boundaries. The particle goes to one boundary, then the other, and finally back to the origina position. (b) The unfolded picture. We have a closed circle. The leftmost solid line is identified the rightmost one. The motion that leads to the Bethe equations involves moving the magnon with momentum $p$ around the closed circle and at the same time we also move its partner which has momentum $-p$ around the circle in the opposite direction.

around the pair with momentum $p_{k}$ and $-p_{k}$ in opposite directions. For level II impurities, we have to collect the factors corresponding to going through all the level I pairs and all the level III impurities (scattering between level II particles is trivial). Finally, for level III impurities we get the factors of going through all the level II impurities and all the other level III impurities (scattering between level III and level I particles is trivial). The resulting set of open Bethe ansatz equations is the following

$$
\begin{aligned}
1= & \left(\frac{x_{k}^{+}}{x_{k}^{-}}\right)^{2 L}\left(\frac{1+\frac{1}{\left(x^{-}\right)^{2}}}{1+\frac{1}{\left(x^{+}\right)^{2}}}\right)^{2} \frac{1}{\sigma_{B}\left(p_{k}\right)^{2} \sigma\left(p_{k},-p_{k}\right)^{2}} \prod_{l=1}^{N^{\mathrm{II}}} \frac{y_{l}-x_{k}^{-}}{y_{l}-x_{k}^{+}} \frac{y_{l}+x_{k}^{-}}{y_{l}+x_{k}^{+}}, \\
& \prod_{l \neq k}^{N^{\mathrm{I}}} \frac{\left(x_{k}^{+}-x_{l}^{-}\right)\left(1-\frac{1}{x_{k}^{-} x_{l}^{+}}\right)}{\left(x_{k}^{-}-x_{l}^{+}\right)\left(1-\frac{1}{x_{k}^{+} x_{l}^{-}}\right)} \frac{\left(x_{l}^{+}+x_{k}^{+}\right)\left(1+\frac{1}{x_{l}^{-} x_{k}^{-}}\right)}{\left(x_{l}^{-}+x_{k}^{-}\right)\left(1+\frac{1}{x_{l}^{+} x_{k}^{+}}\right)} \frac{1}{\sigma\left(p_{k}, p_{l}\right)^{2} \sigma\left(p_{l},-p_{k}\right)^{2}} \\
1= & e^{i \theta-i \phi} \prod_{l=1}^{N^{\mathrm{I}}} \frac{y_{k}-x_{l}^{+}}{y_{k}-x_{l}^{-}} \frac{y_{k}+x_{l}^{-}}{y_{k}+x_{l}^{+}} \prod_{l=1}^{N^{\mathrm{III}}} \frac{w_{l}-v_{k}-\frac{i}{g}}{w_{l}-v_{k}+\frac{i}{g}} \\
1= & e^{-2 i \theta} \prod_{l=1}^{N^{\mathrm{II}}} \frac{w_{k}-v_{l}+\frac{i}{g}}{w_{k}-v_{l}-\frac{i}{g}} \prod_{l \neq k}^{N^{\mathrm{III}}} \frac{w_{k}-w_{l}-\frac{2 i}{g}}{w_{k}-w_{l}+\frac{2 i}{g}} .
\end{aligned}
$$

Eq. (3.9) can be re-written, including $l=k$ in the second product, as

$$
\begin{aligned}
1= & -\left(\frac{x_{k}^{+}}{x_{k}^{-}}\right)^{2 L} \frac{x^{-}+\frac{1}{x^{-}}}{x^{+}+\frac{1}{x^{+}}} \frac{1}{\sigma_{B}\left(p_{k}\right)^{2}} \prod_{l=1}^{N^{\mathrm{II}}} \frac{y_{l}-x_{k}^{-}}{y_{l}-x_{k}^{+}} \frac{y_{l}+x_{k}^{-}}{y_{l}+x_{k}^{+}}, \\
& \prod_{l=1}^{N^{\mathrm{I}}} \frac{\left(x_{k}^{+}-x_{l}^{-}\right)\left(1-\frac{1}{x_{k}^{-} x_{l}^{+}}\right)}{\left(x_{k}^{-}-x_{l}^{+}\right)\left(1-\frac{1}{x_{k}^{+} x_{l}^{-}}\right)} \frac{\left(x_{l}^{+}+x_{k}^{+}\right)\left(1+\frac{1}{x_{l}^{-} x_{k}^{-}}\right)}{\left(x_{l}^{-}+x_{k}^{-}\right)\left(1+\frac{1}{x_{l}^{+} x_{k}^{+}}\right)} \frac{1}{\sigma\left(p_{k}, p_{l}\right)^{2} \sigma\left(p_{l},-p_{k}\right)^{2}}
\end{aligned}
$$


As usual, the energy is given by

$$
\mathcal{E}=\sum_{k=1}^{N^{I}} \epsilon\left(p_{k}\right)
$$

\section{The boundary TBA equations}

The Bethe equations (3.9)-(3.11) presented in the previous section are the correct description of the spectrum for large chains, $L \gg 1$. As $L$ becomes small, wrapping effects come into play and the Bethe equations are no longer valid. Moreover, in this paper, we are mainly interested in $L=0$. A description of the spectrum that is valid for any $L$ is the Boundary Thermodynamic Bethe Ansatz (BTBA) equations. These are a set of integral equations that govern the dynamics in the mirror channel. That is, the dynamics of excitations after exchanging the two dimensional space and time directions [17, 31], see figure 2 . The TBA equations can be derived from the knowledge of the spectrum of states and bound states in the mirror channel. This spectrum was derived in [32]. The derivation of the TBA equations then follows the standard route given in [31, 33-36]. In the case that we have a boundary we can follow essentially the same route. We use the boundary state defined in section 2, and the untangling of boundary reflection matrices described in figure 5. Then we get a TBA which looks very similar to what we would obtain for a closed chain of twice the length $L$, except for the fact that for each particle of momentum $q$ we get one of momentum $-q$, since the boundary state creates such a pair of particles. The consequence of this is that the $Y$ functions obey a reflection property

$$
Y_{a, s}(u)=Y_{a,-s}(-u)
$$

The set of $Y_{a, s}$ functions is the same as the one we have for the closed string problem [3436]. However, due to (4.1) we can restrict our attention to the ones with $s \geq 0$. The boundary data appears as chemical potentials which depend on the angles, $\theta$, $\phi$, as well as a $u$ dependent chemical potential given by the boundary dressing phase $\sigma_{B}$. The precise form of the equations is derived in appendix (E).

Let us summarize the final equations

$$
\begin{aligned}
& \log \frac{Y_{1,1}}{\mathbf{Y}_{1,1}}=K_{m-1} * \log \frac{1+\bar{Y}_{1, m}}{1+\overline{\mathbf{Y}}_{1, m}} \frac{1+\mathbf{Y}_{m, 1}}{1+Y_{m, 1}}+\mathcal{R}_{1 a}^{(01)} * \log \left(1+Y_{a, 0}\right) \\
& \log \frac{\bar{Y}_{2,2}}{\overline{\mathbf{Y}}_{2,2}}=K_{m-1} * \log \frac{1+\bar{Y}_{1, m}}{1+\overline{\mathbf{Y}}_{1, m}} \frac{1+\mathbf{Y}_{m, 1}}{1+Y_{m, 1}}+\mathcal{B}_{1 a}^{(01)} * \log \left(1+Y_{a, 0}\right) \\
& \log \frac{\bar{Y}_{1, s}}{\overline{\mathbf{Y}}_{1, s}}=-K_{s-1, t-1} * \log \frac{1+\bar{Y}_{1, t}}{1+\overline{\mathbf{Y}}_{1, t}}-K_{s-1} \hat{*} \log \frac{1+Y_{1,1}}{1+\bar{Y}_{2,2}} \\
& \log \frac{Y_{a, 1}}{\mathbf{Y}_{a, 1}}=-K_{a-1, b-1} * \log \frac{1+Y_{b, 1}}{1+\mathbf{Y}_{b, 1}}-K_{a-1} \hat{*} \log \frac{1+Y_{1,1}}{1+\bar{Y}_{2,2}} \\
& \quad+\left[\mathcal{R}_{a b}^{(01)}+\mathcal{B}_{a-2, b}^{(01)}\right] * \log \left(1+Y_{b, 0}\right) \\
& \log \frac{Y_{a, 0}}{\mathbf{Y}_{a, 0}}=\left[2 \mathcal{S}_{a b}-\mathcal{R}_{a b}^{(11)}+\mathcal{B}_{a b}^{(11)}\right] * \log \left(1+Y_{b, 0}\right)+2\left[\mathcal{R}_{a b}^{(10)}+\mathcal{B}_{a, b-2}^{(10)}\right]_{\mathrm{sym}}^{*} \log \frac{1+Y_{b, 1}}{1+\mathbf{Y}_{b, 1}}
\end{aligned}
$$




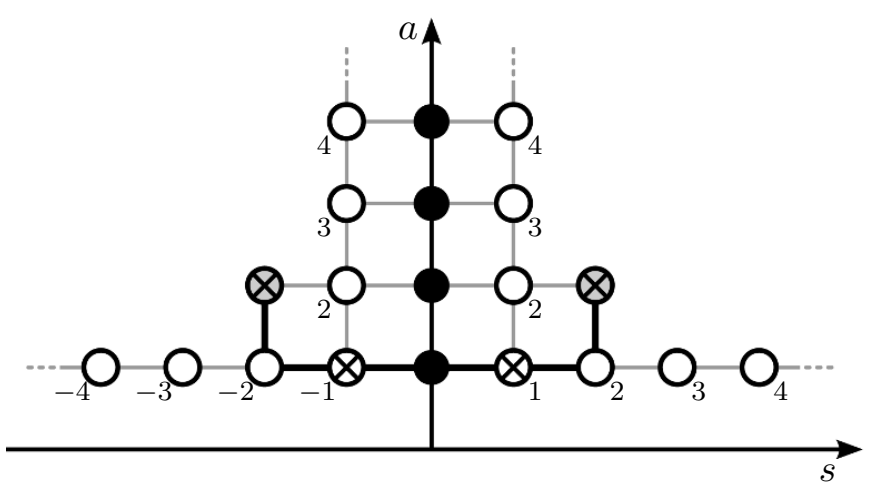

(a)

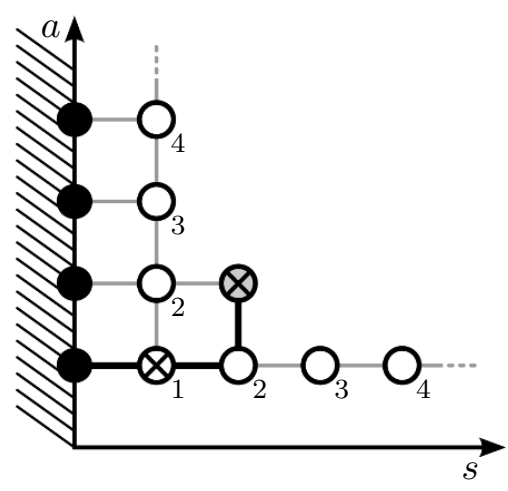

(b)

Figure 8. (a) Set of $Y_{a, s}$ functions for the closed string problem. Here we have the same set but the additional condition (4.1) implies that we can restrict to the set in (b).

$$
+2 \mathcal{R}_{a 1}^{(10)} \underset{\text { sym }}{\hat{*}} \log \frac{1+Y_{1,1}}{1+\mathbf{Y}_{1,1}}-2 \mathcal{B}_{a 1}^{(10)} \underset{\text { sym }}{\hat{*}} \log \frac{1+\bar{Y}_{2,2}}{1+\overline{\mathbf{Y}}_{2,2}}
$$

where we used the conventions of $[37,38]$ for the kernels and integration contours. ${ }^{5}$ We have also defined the barred $Y$ 's as $\bar{Y}_{a, s}^{\text {(here) }}=1 / Y_{a, s}^{\text {(there) }}$, (see appendix E.3 for a summary). Here, the momentum carrying $Y_{a, 0}$ functions are defined as symmetric functions $Y_{a, 0}(-u)=$ $Y_{a, 0}(u)$ and ${ }_{\text {sym }}^{*} f(v)=[* f(v)+* f(-v)] / 2$ is a symmetric convolution. ${ }^{6}$ There are implicit sums over one of the indices of the kernels. ${ }^{7}$ The bold face $\mathbf{Y}$ 's represent the asymptotic large $L$ solution. This is the solution we obtain when the convolutions with the momentum carrying $Y_{a, 0}$ 's are dropped. These asymptotic solutions are the only place where the angles and the boundary dressing phase enter. They are given by

$$
\begin{aligned}
\mathbf{Y}_{1,1} & =-\frac{\cos \theta}{\cos \phi}, \quad \overline{\mathbf{Y}}_{1, s}=\frac{\sin ^{2} \theta}{\sin [(s+1) \theta] \sin [(s-1) \theta]} \\
\overline{\mathbf{Y}}_{2,2} & =-\frac{\cos \theta}{\cos \phi}, \quad \mathbf{Y}_{a, 1}=\frac{\sin ^{2} \phi}{\sin [(a+1) \phi] \sin [(a-1) \phi]} \\
\mathbf{Y}_{a, 0} & =4 \frac{e^{i \chi\left(z^{[+a]}\right)+i \chi\left(1 / z^{[-a]}\right)}}{e^{i \chi\left(z^{[-a]}\right)+i \chi\left(1 / z^{[+a]}\right)}}\left(\frac{z^{[-a]}}{z^{[+a]}}\right)^{2 L+2}(\cos \phi-\cos \theta)^{2} \frac{\sin ^{2} a \phi}{\sin ^{2} \phi} .
\end{aligned}
$$

where $\chi$ is the function defining the boundary dressing phase (2.8). Notice that the length $L$ appears only in (4.9). Here $z^{[ \pm a]}$ are the solutions of

$$
u=g\left(z^{[+a]}+\frac{1}{z^{[+a]}}\right)-i \frac{a}{2}=g\left(z^{[-a]}+\frac{1}{z^{[-a]}}\right)+i \frac{a}{2}=\frac{q}{2} \sqrt{1+\frac{16 g^{2}}{a^{2}+q^{2}}}
$$

\footnotetext{
${ }^{5}$ The convolutions of terms depending on $Y_{1,1}$ or $\bar{Y}_{2,2}$ are over a finite range $|u| \leq 2 g$. We use $\hat{*}$ as a reminder of that.

${ }^{6}$ For the ground state, we expect all functions to be symmetric, $Y_{a, s}(u)=Y_{a, s}(-u)$. But for excited states (4.1) only requires the $Y_{a, 0}$ functions to be symmetric. The equation for excited states could in principle be obtained by analytic continuation from these equations [37-40].

${ }^{7}$ The indices of $Y_{1, m}$ or $Y_{m, 1}$ run over $m \geq 2$. For $Y_{b, 0}$ they run over $b \geq 1$. The same as in [38].
} 
in the mirror region with $\left|z^{[+a]}\right|>1$ and $\left|z^{[-a]}\right|<1$.

Once we solve this system of equations, we can compute the ground state energy as

$$
\mathcal{E}=-\sum_{a=1}^{\infty} \int_{0}^{\infty} \frac{d q}{2 \pi} \log \left(1+Y_{a, 0}\right)
$$

where $q$ is the mirror momentum of each magnon bound state

$$
q=g\left[z^{[+a]}-z^{[-a]}-\frac{1}{z^{[+a]}}+\frac{1}{z^{[-a]}}\right]
$$

\subsection{Recovering the Luscher result}

As a simple check of these equations let us rederive the results of section 2.4. In the large $L$ limit we see that the factor $\left(\frac{z^{[-a]}}{z^{[+a]}}\right)^{2 L+2}=e^{-E_{m} 2(L+1)}$ is very small. This implies that the $\mathbf{Y}_{a, 0}$ in (4.9) are very small. So we expect that the $Y_{a, 0}$ are also small and that we can set them to zero in all the convolution terms of the TBA equations. In this limit, the energy is given by inserting the asymptotic form $\mathbf{Y}_{a, 0},(4.9)$, in the expression for the energy (4.11). One would be tempted to expand the logarithm in (4.11), since $\mathbf{Y}_{a, 0}$ is very small. However, $\mathbf{Y}_{a, 0}$ has a double pole a $u=0$, or $q=0$, coming from the boundary dressing phase. In other words, it behaves as

$$
\mathbf{Y}_{a, 0} \sim \frac{G_{a}^{2}}{q^{2}}+\mathcal{O}(1)
$$

for small $q$. We can then write the integrals in (4.11) as

$$
\int_{0}^{\infty} \frac{d q}{2 \pi} \log \left(1+\mathbf{Y}_{a, 0}\right)=\int_{0}^{\infty} \frac{d q}{2 \pi} \log \left(1+\frac{G_{a}^{2}}{q^{2}}\right)+\int_{0}^{\infty} \frac{d q}{2 \pi} \log \frac{\left(1+\mathbf{Y}_{a, 0}\right)}{\left(1+\frac{G_{a}^{2}}{q^{2}}\right)}
$$

In the second term we can certainly expand to first order in $\mathbf{Y}_{a, 0}$ and $G_{a}^{2}$, which produces a result which is of order $e^{-2 E_{m}(L+1)}$. The first term, however, gives $G_{a} / 2 \sim e^{-E_{m}(L+1)}$, which is bigger. So we get

$$
\mathcal{E} \sim-\frac{1}{2} \sum_{a=1}^{\infty} G_{a}
$$

But this is precisely the same as what we got in section 2. Namely, (2.23) is the same as (4.15) after we realize that $G_{a}$ defined in (4.13) is essentially the same as (2.32), using (4.9). This is not too surprising since [23] derived (2.23) by appealing to TBA equations. In summary, (4.15) agrees precisely with (2.33).

In the next section we will perform a weak coupling check of the equations. We will derive a simplified set of equations that describe the small angle limit $\theta, \phi \sim 0$ and we will expand and solve the resulting equations up to order $g^{6}$. 


\section{The near BPS limit}

When $\phi=\theta$ the Wilson loop is BPS and the energy vanishes. As we deform the angles away from this supersymmetric configuration, the energy behaves as

$$
\Gamma_{\text {cusp }}(\phi, \theta)=-\left(\phi^{2}-\theta^{2}\right) \frac{1}{1-\frac{\phi^{2}}{\pi^{2}}} B(\tilde{\lambda})+\mathcal{O}\left(\left(\phi^{2}-\theta^{2}\right)^{2}\right), \quad \tilde{\lambda}=\lambda\left(1-\frac{\phi^{2}}{\pi^{2}}\right) .
$$

The function $B$, also known as the "Bremsstrahlung function", is related to a variety of physical quantities $[8,9]$. It was computed exactly in $[8,9]$ using localization. In the planar limit we get

$$
B=\frac{1}{4 \pi^{2}} \frac{\sqrt{\tilde{\lambda}} I_{2}(\sqrt{\tilde{\lambda}})}{I_{1}(\sqrt{\tilde{\lambda}})}+\mathcal{O}\left(1 / N^{2}\right)
$$

On the one hand, this allows us to test the BTBA equation to high loop orders by penetrating deep into almost all parts of the equation. On the other hand, the simplicity of (5.2) suggests that, in the near BPS limit, the BTBA equations can be drastically simplified. The equations we will find in this limit are not that simple. We hope that understanding how to simplify them will teach us how to simplify TBA equation in general.

In this section we will study the BTBA equations in this limit. We will show that the BTBA equations can be reduced to a simplified set of equations. We will then solve them to 3 -loop order. Here we restrict the discussion to $\theta=0,{ }^{8}$ so that $\tilde{\lambda}=\lambda$ and $\Gamma_{\text {cusp }}(\phi, \theta)=$ $-\phi^{2} B(\lambda)+\mathcal{O}\left(\phi^{4}\right)$. We also set $L=0$ to extract the cusp anomalous dimension. It is important to note that now $\phi$ is the smallest parameter. In particular, it is smaller than $\lambda$.

In this small angle limit, the momentum carrying Y-functions are of order $Y_{a, 0}=$ $\mathcal{O}\left(\phi^{4}\right)$ and therefore very small. This limit reminds us of the large $L$ asymptotic limit where the momentum carrying $Y_{a, 0}$ 's are exponentially suppressed. However, as opposed to the asymptotic limit, in the small angle limit, we cannot drop the convolutions with the momentum carrying $Y_{a, 0}$ 's. Instead, we remain with a simplified set of non linear equations. The reason is that the large value of $\log Y_{a, 0}$ is not due to the sources in the BTBA equations. Instead, it is due to the fact that the fermionic $Y$-functions $\left(Y_{1,1}\right.$ and $\left.Y_{2,2}\right)$ approach -1 and lead to a big contribution through the $\log \left(1+Y_{1,1}\right)$ and $\log \left(1+Y_{2,2}\right)$ terms in the convolutions.

\subsection{The simplified equations at small angles}

As the momentum carrying Y-functions are small, they only contribute to $B(\lambda)$ through their double pole. We define $\mathbb{C}_{a}$ as the coefficient of the double pole at $u=0$,

$$
\lim _{\substack{q \rightarrow 0 \\ \phi \rightarrow 0}} Y_{a, 0}=\left[-\frac{\phi^{2}}{2 u} \mathbb{C}_{a}\right]^{2}
$$

\footnotetext{
${ }^{8}$ The general near BPS case, with $\theta \neq 0$, has a similar degree of complexity. In fact, we have explicitly expanded the equations up to second order in $\lambda$ and verified the corresponding expansion in (5.2). But we will not give the details here.
} 
The energy, which is dominated by the value of $Y_{a, 0}$ at the double pole, reduces to

$$
\mathcal{E}=\frac{\phi^{2}}{2} \sum_{a=1}^{\infty} \frac{\mathbb{C}_{a}}{\sqrt{1+16 g^{2} / a^{2}}}
$$

where square root factor comes from the $q \rightarrow 0$ limit of $(q / 2 u)$, see (4.10).

In this small $\phi$-limit, the other $Y$-functions can be expanded as

$$
\begin{aligned}
Y_{1,1}=-1-\phi^{2} \Psi+\mathcal{O}\left(\phi^{4}\right), & Y_{m, 1}=\mathcal{Y}_{m}\left[1+\phi^{2}\left(\Omega_{m}-\mathcal{X}_{m}\right) / 2\right]+\mathcal{O}\left(\phi^{4}\right) \\
\bar{Y}_{2,2}=-1-\phi^{2} \Phi+\mathcal{O}\left(\phi^{4}\right), & \bar{Y}_{1, m}=\mathcal{Y}_{m}\left[1+\phi^{2}\left(\Omega_{m}+\mathcal{X}_{m}\right) / 2\right]+\mathcal{O}\left(\phi^{4}\right)
\end{aligned}
$$

where we assumed that to leading order $Y_{1,1}=Y_{2,2}=-1$ and $Y_{m, 1}=Y_{1, m}$. It is not difficult to see that this assumption is consistent with the BTBA equations. Moreover, we find that the functions $\Omega_{m}$ drop out of the equations.

We find that the BTBA equations (4.2)-(4.9) reduce to

$$
\begin{aligned}
\Psi & =\frac{1}{2}+K_{m-1} *\left[\mathcal{X}_{m} \frac{\mathcal{Y}_{m}}{1+\mathcal{Y}_{m}}+\frac{1}{3}\right]-\pi \mathbb{C}_{a} \mathcal{R}_{1 a}^{(01)}(u, 0) \\
\Phi & =\frac{1}{2}+K_{m-1} *\left[\mathcal{X}_{m} \frac{\mathcal{Y}_{m}}{1+\mathcal{Y}_{m}}+\frac{1}{3}\right]-\pi \mathbb{C}_{a} \mathcal{B}_{1 a}^{(01)}(u, 0) \\
\log \mathcal{Y}_{m} & =-K_{m-1, n-1} * \log \left(1+\mathcal{Y}_{n}\right)-K_{m-1} \hat{*} \log \frac{\Psi}{\Phi} \\
\mathcal{X}_{m} & =-\frac{m^{2}}{3}-K_{m-1, n-1} *\left[\mathcal{X}_{n} \frac{\mathcal{Y}_{n}}{1+\mathcal{Y}_{n}}+\frac{1}{3}\right]+\pi \mathbb{C}_{n}\left[\mathcal{R}_{m n}^{(01)}+\mathcal{B}_{m-2, n}^{(01)}\right](u, 0) \\
\Delta_{\mathrm{conv}} & =\left.\left\{\mathcal{R}_{a 1}^{(10)} \hat{*} \log \left(\frac{\Psi}{1 / 2}\right)-\mathcal{B}_{a 1}^{(10)} * \log \left(\frac{\Phi}{1 / 2}\right)+\left[\mathcal{R}_{a b}^{(10)}+\mathcal{B}_{a, b-2}^{(10)}\right] * \log \left(\frac{1+\mathcal{Y}_{b}}{1+\frac{1}{b^{2}-1}}\right)\right\}\right|_{u=} \\
\mathbb{C}_{a} & =(-1)^{a} a^{2} F(a, g) \frac{z_{0}^{[-a]}}{z_{0}^{[+a]}} e^{\Delta_{\text {conv }}}
\end{aligned}
$$

where $z_{0}^{[ \pm a]}$ denote the values of $z^{[ \pm a]}$ at $q=0$ (2.16). In (5.11) we are evaluating the non-convoluted variable of the kernels at $u=0$. The hat on $\hat{*}$ is a convolution over the range $|u| \leq 2 g . F(a, g)$ is given in (2.31). These equations are derived by implementing the expansion of Y-functions (B.18) in the TBA system of equations (4.2)-(4.6). Let us make a couple of comments. First, the factors of $1 / 2,1 / 3, \mathrm{~m}^{2} / 3$

stand for the subtraction of the asymptotic solutions. These read

$$
\underline{\Psi}=\underline{\Phi}=\frac{1}{2}, \quad \underline{\mathcal{Y}_{m}}=\frac{1}{m^{2}-1}, \quad \underline{\mathcal{X}_{m}}=-\frac{m^{2}}{3}
$$

Second, note that in the BPS vacuum where $\phi=0$, the TBA equations are not well defined and need a regularization. A regulator commonly used is a twist for the fermions [41]. Here, the angle $\phi$ can be viewed as a physical regulator. As opposed to other regulators, the leading order solution $\mathcal{Y}_{m}$ is a non trivial function of the coupling. 


\subsection{Weak coupling expansion of the small $\phi$ TBA}

To test the BTBA equations, we have solved the small angle simplified equations, (5.6)(5.12), up to three loops. In this section we will present the results. The derivation is given in appendix $\mathrm{F}$.

The small $\phi$ TBA equations, (5.6)-(5.12), are certainly simpler than the general TBA equations (4.2)-(4.6), but they continue to be non-linear. However, if we make a weak coupling expansion we obtain a linear system of integral equations order by order.

To solve these linear equations we find it useful to first simplify the TBA equations as in $[42,43]$. To simplify (5.6) and (5.7), we take a convolution of the equations with $s * s^{-1}$ where

$$
s(u)=\frac{1}{2 \cosh (\pi u)}
$$

The other equations can also be simplified as shown in the appendix F. Then (5.6)-(5.12) become

$$
\begin{aligned}
\Phi-\Psi & =\pi \mathbb{C}_{a} \hat{K}_{y, a}(u, 0) \\
\Phi+\Psi & =-2 s * \frac{\mathcal{X}_{2}}{1+\mathcal{Y}_{2}}+2 \pi s * \mathcal{R}_{2 n}^{(01)}(u, 0) \mathbb{C}_{n}-\pi \mathbb{C}_{a} K_{a}(u, 0) \\
\log \mathcal{Y}_{m} & =s * I_{m, n} \log \frac{\mathcal{Y}_{n}}{1+\mathcal{Y}_{n}}+\delta_{m, 2} s \hat{*} \log \frac{\Phi}{\Psi} \\
\mathcal{X}_{m} & =s * I_{m, n} \frac{\mathcal{X}_{n}}{1+\mathcal{Y}_{n}}+\pi s \mathbb{C}_{m}+\delta_{m, 2} s \hat{*}(\Phi-\Psi) \\
\Delta_{\text {conv }} & =\left.\left\{\mathcal{R}_{a 1}^{(10)} * \log \left(\frac{\Psi}{1 / 2}\right)-\mathcal{B}_{a 1}^{(10)} * \log \left(\frac{\Phi}{1 / 2}\right)+\left[\mathcal{R}_{a b}^{(10)}+\mathcal{B}_{a, b-2}^{(10)}\right] * \log \left(\frac{1+\mathcal{Y}_{b}}{1+\frac{1}{b^{2}-1}}\right)\right\}\right|_{u=} \\
\mathbb{C}_{a} & =(-1)^{a} a^{2} F(a, g) \frac{z_{0}^{[-a]}}{z_{0}^{[+a]}} e^{\Delta_{\text {conv }}}
\end{aligned}
$$

where $I_{m, n}=\delta_{m+1, n}+\delta_{m-1, n}$ and $\hat{K}_{y, a}$ is defined in appendix F.

Now expanding the functions $\Psi, \Phi, \mathcal{Y}_{n}$ and $\mathcal{X}_{n}$ in powers of $g^{2}$, we can obtain them order by order by solving a linear system of equations. Up to three loops (see appendix $\mathrm{F}$ for details) we find that

$$
\mathbb{C}_{a}=4(-1)^{a} g^{2}+8(-1)^{a}\left[\pi^{2}-\frac{4}{a^{2}}\right] g^{4}+16(-1)^{a}\left[\frac{\pi^{4}}{3}-\frac{4 \pi^{2}}{a^{2}}+\frac{20}{a^{4}}\right] g^{6}+\mathcal{O}\left(g^{8}\right),
$$

Finally, the relation (5.4), we obtain the expression for energy up to 3-loop order ${ }^{9}$

$$
\mathcal{E}=-\phi^{2}\left[g^{2}-g^{4} \frac{2 \pi^{2}}{3}+g^{6} \frac{2 \pi^{4}}{3}+O\left(g^{8}\right)\right]=-\phi^{2}\left[\frac{\lambda}{16 \pi^{2}}-\frac{\lambda^{2}}{384 \pi^{2}}+\frac{\lambda^{3}}{6144 \pi^{2}}+\mathcal{O}\left(\lambda^{4}\right)\right],
$$

In perfect agreement with the expansion of (5.2).

\footnotetext{
${ }^{9}$ We encounter the sum $\sum_{a=1}^{\infty}(-1)^{a}=-\frac{1}{2}$. This can be understood by regularizing it as $\lim _{\phi \rightarrow 0}\left[\sum_{a=1}^{\infty}(-1)^{a} \frac{\sin a \phi}{a \phi}\right]=-\frac{1}{2}$.
} 


\section{Conclusions and discussion}

In this paper we have considered the problem of computing the quark anti-quark potential on the 3 -sphere in $\mathcal{N}=4$ super Yang Mills in the planar approximation. Since the planar theory is integrable [12], we expected to be able to derive an exact expression. Indeed, we found a system of boundary TBA equations (4.2)-(4.6) which determines the potential as a function of three parameters: the planar coupling $\lambda$, the geometric angle $\phi$, which sets the angular separation on the 3 -sphere and an internal angle $\theta$ which is the relative orientation of the coupling to the scalar field for the quark and the anti-quark.

This quark and anti-quark configuration gives rise to an integrable system with a boundary. This is most clearly seen in the string theory picture where we have a string going between the two lines on the boundary. One might be surprised that we have a boundary since the string is infinitely long. However, note that the local geometry of the string near the boundary is $A d S_{2}$, which indeed has a boundary. The energy is then the ground state energy, or Casimir energy, on the strip and it is given in terms of the solution of the TBA equations (4.11). This is the energy of the flux tube connecting the quark and anti-quark. These TBA equations should also enable one to compute the energies of excitations of the flux tube. These correspond to operators that are inserted on the Wilson loop.

The quark anti-quark potential on $S^{3}$ is the same as the cusp anomalous dimension as a function of the angles, $\Gamma_{\text {cusp }}(\phi, \theta, \lambda)$.

The derivation of the boundary TBA equations is similar to the one in other integrable models with boundary [18]. A crucial step is the determination of the boundary reflection matrix. The matrix part is fixed by the symmetries and the dressing phase was found by solving the boundary crossing equation and the final answer is in (2.8), (2.9). Since there is always a certain amount of guesswork in determining the dressing phase, we have checked it at strong coupling and we have seen that it gives the right value both in the physical and mirror regions. A crucial feature of the dressing phase is that it contains a pole at zero mirror momentum. This is crucial for the proposed phase to work at weak coupling. Note that the boundary dressing phase is responsible for the leading order contribution in the mirror picture, while it only starts contributing at three loops for anomalous dimensions in the physical picture. The pole simply means that the boundary is sourcing single particle states.

The BTBA equations were written in (4.2)-(4.6). They look very similar to the bulk TBA equations [34, 36, 42], except that the boundary conditions for large $u$ are different. They now depend on the angles. In addition, for the momentum carrying nodes, the $Y_{a, 0}$, there is an extra source term involving the boundary dressing phase.

We have obtained a simplified set of equations, (5.15)-(5.20), which describes the small angle region, $\phi, \theta \ll 1$. In this region, the simplest way to solve the problem is through supersymmetric localization, as explained in [8]. The planar answer is

$$
\Gamma_{\text {cusp }}(\phi, \theta=0, \lambda)=-\phi^{2} B+\mathcal{O}\left(\phi^{4}\right), \quad B=\frac{1}{4 \pi^{2}} \frac{\sqrt{\lambda} I_{2}(\sqrt{\lambda})}{I_{1}(\sqrt{\lambda})}
$$

So, we know the answer by independent means. Thus, these simplified BTBA equations should reproduce (6.1). Indeed, directly expanding these simplified equations up to third 
order in the coupling we reproduced the expansion of (6.1). However, these "simplified" equations are vastly more complex than the simple Bessel functions in (6.1)!. Thus, there should be a way to simplify these equations much further and directly get the simple answer (6.1). Hopefully, the methods used to simplify the equation will also be useful in order to simplify the full BTBA equations for general angles. Note that in [44] the TBA system for closed strings was reduced to a set of equations involving a finite number of functions. It is very likely that the same method works in our case.

Notice that the simplified small angle equations connect the integrability and the localization exact solutions. In particular, computing the function $B$ by both methods would enable us to see whether the coupling constant $\lambda$ that appears in both approaches is the same or not. Of course, we expect them to be the same for $\mathcal{N}=4$ super Yang Mills. However, if one could generalize the discussion in this paper to Wilson loops in ABJM theory [45], then this small angle region could enable us to compute the undetermined function $h(\lambda)$ that appears in the integrability approach to the ABJM theory [46].

In principle, one might wonder whether the Wilson loop leads to an integrable boundary condition. We have found that the reflection matrix obeys the boundary Yang Baxter equation. The TBA equations were derived assuming integrability. So all the checks we performed on them are further evidence that the Wilson loop boundary condition is indeed integrable.

There are further checks of the equations that one should be able to do. In particular, one would like to reproduce the BES equation [6] for $\varphi \rightarrow \infty$.

It would also be nice to take the small $\delta=\pi-\phi$ limit. In this limit the answer should go like $1 / \delta$ and probably one can obtain again a simplified equation for the coefficient. This determines the quark anti-quark potential in the flat space limit.

One should also be able to take the strong coupling limit of the equations and reproduce the result derived from classical strings in $A d S_{5} \times S^{5}$ in $[47,48]$. It is likely that the ideas in $[49,50]$ would enable this.

Though solving the TBA equation analytically looks difficult, it should be possible to solve the equations numerically. The problem should be very similar to the one solved in [51].

It would also be nice to study the problem of determining the open string spectrum on the $A d S_{4} \times S^{2}$ or $A d S_{2} \times S^{4}$ D-branes which also preserve the same amount of symmetry. The only difference with the current paper should be a different choice for the boundary dressing phase. For this reason, the TBA equations would be the same, except for the choice of the boundary dressing phase.

The study of perturbative amplitudes at weak coupling has found remarkably simple underlying structures. It would be interesting to study these structures in the context of the cusp anomalous dimension, where we have a function of a single angle $\phi$. In particular, it would be nice to see how to connect those structures with the TBA approach described here. This would most probably lead to both a simplification of this TBA approach as well as some hints on the exact structure underlying the amplitude problem.

Throughout this paper we have considered the locally BPS Wilson loop which contains the coupling to the scalar, as in (1.2). Of course, one can also consider the Wilson loop 
which does not couple to the scalars, $W=\operatorname{tr} P e^{i \oint A}$. It would be interesting to see whether this leads to an integrable boundary condition. At strong coupling this loop leads to a Neumann boundary condition on the $S^{5}$ [52], which is classically integrable. ${ }^{10}$

Note. We were informed that similar ideas were pursued in [24].

\section{Acknowledgments}

We would like to thank N. Arkani-Hamed, B. Baso, S. Caron-Huot, N. Drukker, D. Gaiotto, N. Gromov, I. Klebanov, P. Vieira and A. Zamolodchikov for discussions.

A. S. would like to thank Nordita for warm hospitality. This work was supported in part by U.S. Department of Energy grant \#DE-FG02-90ER40542. Research at the Perimeter Institute is supported in part by the Government of Canada through NSERC and by the Province of Ontario through MRI. The research of A.S. has been supported in part by the Province of Ontario through ERA grant ER 06-02-293. D.C would like to thanks IAS for hospitality. The research of D.C has been supported in part by a CONICET-Fulbright fellowship and grant PICT 2010-0724.

\section{A Reflection matrix}

With the conventions we are using, the (canonical) diagonal symmetry generators are

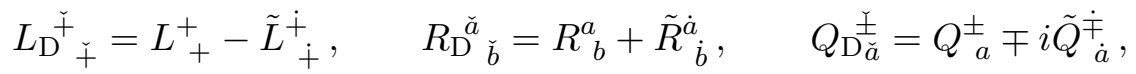

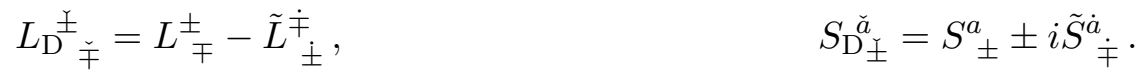

The generators (A.1) give rise to the diagonal $\widetilde{s u}(2 \mid 2)_{\mathrm{D}}$ residual symmetry. Now we should determine how a bulk magnon transforms under the diagonal $\widetilde{s u}(2 \mid 2)_{\mathrm{D}}$. A bulk magnon transforms in a representation $\left(\square_{(a, b, c, d)}, \tilde{\square}_{(a, b, c, d)}\right)$ of the bulk symmetry $\widetilde{s u}(2 \mid 2)_{L} \times$ $\widetilde{s u}(2 \mid 2)_{R}$. The quantum numbers

$$
a=\sqrt{g} \eta, \quad b=\sqrt{g} \frac{i \zeta}{\eta}\left(\frac{x^{+}}{x^{-}}-1\right), \quad c=-\sqrt{g} \frac{\eta}{\zeta x^{+}}, \quad d=-\sqrt{g} \frac{x^{+}}{i \eta}\left(\frac{x^{-}}{x^{+}}-1\right),
$$

characterize the action of the fermionic generators. For the left fundamental $\left(\phi^{1}, \phi^{2}, \psi^{+}, \psi^{-}\right)$

$$
\begin{aligned}
Q_{a}^{\alpha}\left|\phi^{b}\right\rangle & =a \delta_{a}^{b}\left|\psi^{\alpha}\right\rangle, & S_{\alpha}^{a}\left|\phi^{b}\right\rangle & =c \epsilon_{\alpha \beta} \epsilon^{a b}\left|\psi^{\beta}\right\rangle, \\
Q_{a}^{\alpha}\left|\psi^{\beta}\right\rangle & =b \epsilon^{\alpha \beta} \epsilon_{a b}\left|\phi^{b}\right\rangle, & S_{\alpha}^{a}\left|\psi^{\beta}\right\rangle & =d \delta_{\alpha}^{\beta}\left|\phi^{a}\right\rangle,
\end{aligned}
$$

and similarly for the right generators $\tilde{Q}_{\dot{a}}^{\dot{\alpha}}$ and $\tilde{S}_{\dot{\alpha}}^{\dot{a}}$ and on a right fundamental $\left(\tilde{\phi}^{\dot{1}}, \tilde{\phi}^{\dot{2}}, \tilde{\psi}^{\dot{+}}, \tilde{\psi}^{-}\right) . \zeta$ is a phase and unitarity requires $|\eta|^{2}=i\left(x^{-}-x^{+}\right)$.

The left part of a bulk magnon, $\nabla_{(a, b, c, d)}$ is also $\nabla_{(a, b, c, d)}^{\mathrm{D}}$, with identical quantum numbers However, the right $\tilde{\nabla}_{(a, b, c, d)}$ needs to be re-arranged to transform canonically under the action of the diagonal symmetry generators. That can be achieved defining,

$$
\left(\tilde{\phi}^{\check{1}}, \tilde{\phi}^{\check{2}}, \tilde{\psi}^{\check{+}}, \tilde{\psi}^{-}\right):=\left(\tilde{\phi}^{\dot{1}}, \tilde{\phi}^{\dot{2}},-i \tilde{\psi}^{-},+i \tilde{\psi}^{\dot{+}}\right),
$$

\footnotetext{
${ }^{10}$ See [61] for a systematic study of classically integrable boundary conditions.
} 
which turns out to be a $\nabla_{(a,-b,-c, d)}^{\mathrm{D}}$. Due to the signs in $-b$ and $-c$ we should interpret the right part as a magnon with quasi-momentum $-p$ and phase $\zeta e^{i p}$ under $s u(2 \mid 2)_{\mathrm{D}}$. Therefore, with the change of basis (A.4), the original left and right parts of the bulk magnon transforms in the following tensor representation of $s u(2 \mid 2)_{\mathrm{D}}$

$$
\square_{(a, b, c, d)} \otimes \square_{(a,-b,-c, d)}=\mathcal{V}(p, \zeta) \otimes \mathcal{V}\left(-p, \zeta e^{i p}\right) .
$$

Diagonal $\widetilde{s u}(2 \mid 2)_{\mathrm{D}}$ is preserved during the reflection, which fixes the boundary scattering matrix up to a phase factor. We should take into account that the multiplet labels change with the reflection according to,

$$
\mathcal{V}(p, \zeta) \otimes \mathcal{V}\left(-p, \zeta e^{i p}\right) \rightarrow \mathcal{V}(-p, \zeta) \otimes \mathcal{V}\left(p, \zeta e^{-i p}\right)
$$

The reflection matrix $\mathcal{R}_{\mathrm{R}}(p,-p)$ intertwines the same representations as a bulk $S$-matrix $\mathcal{S}(p,-p)$, and therefore the two must be equal up to a phase [16]. The resulting reflection matrix is given by

$$
\begin{aligned}
\mathcal{R}_{\mathrm{R}}\left|\phi_{p}^{\check{a}} \times \tilde{\phi}_{-p}^{\check{b}}\right\rangle & =A_{\mathrm{R}}(p)\left|\phi_{-p}^{\{\check{a}} \times \tilde{\phi}_{p}^{\check{b}\}}\right\rangle+B_{\mathrm{R}}(p)\left|\phi_{-p}^{[\check{a}} \times \tilde{\phi}_{p}^{\check{b}]}\right\rangle+\frac{1}{2} C_{\mathrm{R}}(p) \epsilon^{\check{a} \check{b}} \epsilon_{\check{\alpha} \check{\beta}}\left|\psi_{-p}^{\check{\alpha}} \times \tilde{\psi}_{p}^{\check{\beta}}\right\rangle, \\
\mathcal{R}_{\mathrm{R}}\left|\psi_{p}^{\check{\alpha}} \times \tilde{\psi}_{-p}^{\check{\beta}}\right\rangle & =D_{\mathrm{R}}(p)\left|\psi_{-p}^{\{\check{\alpha}} \times \tilde{\psi}_{p}^{\check{\beta}\}}\right\rangle+E_{\mathrm{R}}(p)\left|\psi_{-p}^{[\check{\alpha}} \times \tilde{\psi}_{p}^{\check{\beta}]}\right\rangle+\frac{1}{2} F_{\mathrm{R}}(p) \epsilon_{\tilde{a}_{\check{b}}} \epsilon^{\check{\alpha} \check{\beta}}\left|\phi_{-p}^{\check{a}} \times \tilde{\phi}_{p}^{\check{b}}\right\rangle, \\
\mathcal{R}_{\mathrm{R}}\left|\phi_{p}^{\check{a}} \times \tilde{\psi}_{-p}^{\check{\beta}}\right\rangle & =G_{\mathrm{R}}(p)\left|\psi_{-p}^{\check{\beta}} \times \tilde{\phi}_{p}^{\check{a}}\right\rangle+H_{\mathrm{R}}(p)\left|\phi_{-p}^{\check{a}} \times \tilde{\psi}_{p}^{\check{\beta}}\right\rangle, \\
\mathcal{R}_{\mathrm{R}}\left|\psi_{p}^{\check{\alpha}} \times \tilde{\phi}_{-p}^{\check{b}}\right\rangle & =K_{\mathrm{R}}(p)\left|\psi_{-p}^{\check{\alpha}} \times \tilde{\phi}_{p}^{\check{b}}\right\rangle+L_{\mathrm{R}}(p)\left|\phi_{-p}^{\check{b}} \times \tilde{\psi}_{p}^{\check{\alpha}}\right\rangle .
\end{aligned}
$$

where

$$
\begin{array}{ll}
A_{\mathrm{R}}=R_{0}(p) \frac{x^{-}}{x^{+}} \frac{\eta_{1} \eta_{2}}{\tilde{\eta}_{1} \tilde{\eta}_{2}}, & D_{\mathrm{R}}=-R_{0}(p), \\
B_{\mathrm{R}}=-R_{0}(p) \frac{x^{-}\left(x^{-}+\left(x^{+}\right)^{3}\right)}{\left(x^{+}\right)^{2}\left(1+x^{-} x^{+}\right)} \frac{\eta_{1} \eta_{2}}{\tilde{\eta}_{1} \tilde{\eta}_{2}}, & E_{\mathrm{R}}=R_{0}(p) \frac{x^{+}+\left(x^{-}\right)^{3}}{x^{-}\left(1+x^{-} x^{+}\right)}, \\
C_{\mathrm{R}}=-R_{0}(p) \frac{i \eta_{1} \eta_{2}\left(x^{-}+x^{+}\right)}{\zeta x^{+}\left(1+x^{-} x^{+}\right)}, & F_{\mathrm{R}}=-R_{0}(p) \frac{i \zeta\left(x^{-}+x^{+}\right)\left(x^{-}-x^{+}\right)^{2}}{\tilde{\eta}_{1} \tilde{\eta}_{2} x^{+}\left(1+x^{-} x^{+}\right)}, \\
G_{\mathrm{R}}=R_{0}(p) \frac{x^{-}+x^{+}}{2 x^{+}} \frac{\eta_{1}}{\tilde{\eta}_{1}}, & H_{\mathrm{R}}=R_{0}(p) \frac{x^{-}-x^{+}}{2 x^{+}} \frac{\eta_{1}}{\tilde{\eta}_{2}}, \\
K_{\mathrm{R}}=R_{0}(p) \frac{x^{-}-x^{+}}{2 x^{+}} \frac{\eta_{2}}{\tilde{\eta}_{1}}, & L_{\mathrm{R}}=R_{0}(p) \frac{x^{-}+x^{+}}{2 x^{+}} \frac{\eta_{2}}{\tilde{\eta}_{2}} .
\end{array}
$$

$\tilde{\eta}_{i}$ are the values of $\eta_{i}$ after the reflection. The choice for $\eta$ in the so-called string theory basis is

$$
\eta(p, \zeta)=\zeta^{\frac{1}{2}} e^{\frac{i p}{4}} \sqrt{i x^{-}-i x^{+}}
$$

\section{B Solution to the boundary crossing equation}

In this appendix we solve the crossing equation for the boundary dressing phase

$$
\sigma_{B}(p) \sigma_{B}(\bar{p})=\frac{x^{-}+\frac{1}{x^{-}}}{x^{+}+\frac{1}{x^{+}}}, \quad \sigma_{B}(p) \sigma_{B}(-p)=1 .
$$


We follow a procedure similar to the one described in [21, 22]. First we introduce the Zhukovski variable $u$,

$$
x(u)+\frac{1}{x(u)}=\frac{u}{g}, \quad x^{ \pm}:=x\left(u \pm \frac{i}{2}\right),
$$

so that the crossing equation can be written as

$$
\sigma_{B}(u) \sigma_{B}^{\gamma}(u)=\frac{u-\frac{i}{2}}{u+\frac{i}{2}} .
$$

The index $\gamma$ means the analytical continuation along a closed contour $\gamma$ that crosses both $x^{+}(u)$ and $x^{-}(u)$ cuts. We are also going to assume that

$$
\sigma_{B}\left(x^{+}(u), x^{-}(u)\right)=\frac{G\left(x^{+}\right)}{G\left(x^{-}\right)} .
$$

In terms of the shift operator $D:=e^{\frac{i}{2} \partial_{u}}$, the crossing equation reads

$$
[G(x(u)) G(1 / x(u))]^{D-D^{-1}}=u^{D^{-1}-D} .
$$

Therefore, our crossing equation is of the form

$$
G(x(u)) G(1 / x(u))=u^{F(D)} .
$$

Naively one would say that $F(D)=-1$, but that would associate the cuts of $x(u)$ to $G(x(u)) G(1 / x(u))$. Instead, we will use

$$
F_{k}(D)=\frac{D^{k}}{1-D^{k}}+\frac{D^{-k}}{1-D^{-k}}=\sum_{n=1}^{\infty} D^{k n}+\sum_{n=1}^{\infty} D^{-k n},
$$

for some integer $k$. Different values of $k$ would lead to different expressions for $u^{F_{k}(D)}$. A posteriori we will analyze what values of $k$ are consistent with the crossing condition. With this $F_{k}(D)$, we get

$$
u^{F_{k}(D)}=\exp \left(\sum_{n=1}^{\infty} \log \left(u^{2}+\frac{k^{2} n^{2}}{4}\right)\right) .
$$

As in [22], we regulate the divergent sum in (B.8), by taking the derivative of the exponential's argument, performing the sum and integrating back. As a result, up to an irrelevant integration constant, we obtain

$$
u^{F_{k}(D)}=\frac{\sinh \left(\frac{2 \pi}{k} u\right)}{\frac{2 \pi}{k} u}=\frac{1}{\Gamma\left(1+\frac{2 u i}{k}\right) \Gamma\left(1-\frac{2 u i}{k}\right)} .
$$

We should now check consistency with original crossing equation, so we compute:

$$
\left(\frac{\sinh \left(\frac{2 \pi}{k} u\right)}{\frac{2 \pi}{k} u}\right)^{D-D^{-1}}=\frac{\sinh \left(\frac{2 \pi}{k}\left(u+\frac{i}{2}\right)\right)}{\sinh \left(\frac{2 \pi}{k}\left(u-\frac{i}{2}\right)\right)} \frac{u-\frac{i}{2}}{u+\frac{i}{2}} .
$$


Consistency with the crossing condition (B.3) requires

$$
\frac{\sinh \left(\frac{2 \pi}{k}\left(u+\frac{i}{2}\right)\right)}{\sinh \left(\frac{2 \pi}{k}\left(u-\frac{i}{2}\right)\right)}=1,
$$

and we then choose the value $k=1$.

We still need to solve for the function $G(x)$. Let us define $G(x):=e^{i \chi(x)}$. The crossing condition (B.6) imposes

$$
\chi(x(u+i 0))+\chi(x(u-i 0))=\frac{1}{i} \log \left(\frac{\sinh (2 \pi u)}{2 \pi u}\right) .
$$

The kernel introduced in the bulk case can also be used to solve our problem. Indeed, the kernel

$$
K \star f:=\int_{-2 g+i 0}^{2 g+i 0} \frac{d w}{2 \pi i} \frac{x(u)-\frac{1}{x(u)}}{x(w)-\frac{1}{x(w)}} \frac{1}{w-u} f(w),
$$

satisfies

$$
(K \star f)(u+i 0)+(K \star f)(u-i 0)=f(u),
$$

if $|u|<2 g$. Thus, equation (B.12) is solved by

$$
\chi(x(u))=-i K \star \log \left(\frac{\sinh (2 \pi u)}{2 \pi u}\right) .
$$

Up to a term that cancels when we compute the difference $\chi\left(x^{+}\right)-\chi\left(x^{-}\right)$, we can write $\chi(x)$ as a contour integral,

$$
\chi(x)=\Phi(x)=-i \oint_{|z|=1} \frac{d z}{2 \pi i} \frac{1}{x-z} \log \left(\frac{\sinh \left[2 \pi g\left(z+\frac{1}{z}\right)\right]}{2 \pi g\left(z+\frac{1}{z}\right)}\right) .
$$

This 'DHM' representation [53] of the solution for the crossing equation is valid for $|x|>1$. As $x$ moves towards the interior of the unit circle, the function defined by the contour integral is discontinuous, as it picks up a residue at $x$. Since we want the analytic continuation to the interior of the disk to be continuous, for $|x|<1$, we have instead

$$
\chi(x)=\Phi(x)-i \log \left(\frac{\sinh \left[2 \pi g\left(x+\frac{1}{x}\right)\right]}{2 \pi g\left(x+\frac{1}{x}\right)}\right) .
$$

This analogous to the analysis of [54] for the bulk dressing phase. As $x$ moves inside the unit disk, some of the branch cuts of the logarithmic term in (B.17) can be crossed. There are infinitely many of those branch cuts, corresponding to the zeros of $\sinh \left(2 \pi g\left(x+\frac{1}{x}\right)\right)$. However, crossing such branch cuts could only produce $2 \pi$ terms in $\chi$ which are, in any case, irrelevant to the boundary dressing factor $\sigma_{B}\left(x^{+}, x^{-}\right)=e^{i \chi\left(x^{+}\right)-i \chi\left(x^{-}\right)}$.

Being careful about the crossing contour one can check that the final $\sigma_{B}$ obtained after this procedure indeed solve the crossing equation (B.1). The unitarity condition is a 
consequence of the fact that $\chi(-x)=\chi(x)$ plus the the fact that $\sigma_{B}$ is a ratio of a function of $x^{+}$and the same function of $x^{-}$(B.4).

We can expand the contour integral $\Phi(x)$ in negative powers of $x$ for large $|x|$ values,

$$
\Phi(x)=-\sum_{r=1}^{\infty} \frac{c_{r}(g)}{x^{r}} .
$$

The coefficients $c_{r}(g)$ can be expanded either in the weak or in the strong coupling limit. In the weak coupling limit we obtain

$$
c_{r}(g)=\sum_{n=1}^{\infty} \frac{i(-4)^{n} g^{2 n} \zeta(2 n) \Gamma\left(-\frac{r}{2}-n\right)}{\Gamma(1-2 n) \Gamma\left(-\frac{r}{2}+n+1\right)} .
$$

Notice that for odd $r$ the coefficient $c_{r}(g)$ is vanishing, while for even $r$ is order $g^{r}$.

In the strong coupling limit we find

$$
c_{r}(g)=2 g i \int_{0}^{2 \pi} d t e^{i t r}|\cos t|+\mathcal{O}(1)=\frac{4 g i^{r+1}\left(1+(-1)^{r}\right)}{1-r^{2}}+\mathcal{O}(1) .
$$

To evaluate the contour integral for $|x|<1$ we can use the identity

$$
\Phi(x)+\Phi(1 / x)=\Phi(0), \quad \text { for }|\mathrm{x}| \neq 1,
$$

and the expansion

$$
\Phi(0)=-i \sum_{n=1}^{\infty} g^{2 n}(-16)^{n} \frac{(2 n-1) ! !}{n(2 n) ! !} \zeta(2 n)
$$

\section{Luscher correction at strong coupling}

In this appendix we consider an open string operator of the form $B_{l} Z^{L} B_{r}(\theta, \phi)$ and compute the leading correction to the energy for large $L$, this correction goes as $e^{- \text {(constant) } L}$. We will compute the correction for $\phi=0, \theta \neq 0$ at leading order in the strong coupling expansion. In this case we have a string that moves on $A d S_{2} \times S^{3}$. It is convenient to decouple the $A d S_{2}$ and $S^{3}$ problems by choosing a worldsheet gauge where $-T_{ \pm \pm}^{A d S}=T_{ \pm \pm}^{S}=1$. We fix the solution on the $A d S_{2}$ part. This $A d S_{2}$ solution is completely characterized by the extent of the spatial worldsheet coordinate $\sigma$, which we take to run between $[-s / 2, s / 2]$. The other worldsheet coordinate is $\tau$. In particular the spacetime energy $\Delta$ of the solution is fixed, once $s$ is fixed. As we vary the parameters we will see that $L$ will change, $\theta$ will change, and so will $\Delta-L$.

So we now concentrate on the solution on the $S^{3}$, which we parametrize as

$$
x_{1}+i x_{2}=e^{i \gamma \tau} \sqrt{1-\rho^{2}(\sigma)}, \quad x_{3}+i x_{4}=\rho(\sigma) e^{i \varphi(\sigma)}
$$

Inserting this in the Euler Lagrange equations for the string and imposing the Virasoro constraints, $T_{ \pm \pm}=1$ one finds two integrals of motion, $\ell$ and $\gamma$. They are given by

$$
\ell=\rho^{2} \varphi^{\prime}
$$


and

$$
\frac{\rho^{2}\left(\rho^{\prime}\right)^{2}}{1-\rho^{2}}=-\ell^{2}-\left(\gamma^{2}-1\right) \rho^{2}+\gamma^{2} \rho^{4}
$$

The boundary conditions are $\rho^{\prime}(0)=0, \rho(s / 2)=1$. Let us define $\rho_{0}$ to be the value of $\rho$ at $\sigma=0$ where the derivative vanishes. It is a root of

$$
0=-\ell^{2}-\left(\gamma^{2}-1\right) \rho_{0}^{2}+\gamma^{2} \rho_{0}^{4}
$$

By using (C.3) we can write the following expressions

$$
\begin{aligned}
\frac{s}{2} & =\int_{\rho_{0}}^{1} d \rho \frac{\rho}{\sqrt{1-\rho^{2}} \sqrt{D}} \\
\frac{\theta}{2} & =\int_{\rho_{0}}^{1} d \rho \frac{\ell}{\rho \sqrt{1-\rho^{2}} \sqrt{D}} \\
\frac{L}{2} & =2 g \int_{0}^{s / 2} d \sigma \gamma\left|x_{1}+i x_{2}\right|^{2}=2 g \gamma \int_{\rho_{0}}^{1} d \rho \frac{\rho \sqrt{1-\rho^{2}}}{\sqrt{D}} \\
D & =-\ell^{2}-\left(\gamma^{2}-1\right) \rho^{2}+\gamma^{2} \rho^{4}=\left(\rho^{2}-\rho_{0}^{2}\right)\left[\gamma^{2}\left(\rho^{2}+\rho_{0}^{2}\right)-\left(\gamma^{2}-1\right)\right]
\end{aligned}
$$

From the first two equations we should find $\rho_{0}$ and $\gamma$ as a function of $s$ and $\theta$, and then we can find the expression for $L$ and for the energy. We want to find a solution where $L$ is very large.

This happens when $\rho_{0} \rightarrow 0$ and $\gamma \rightarrow 1$ and $\ell \rightarrow 0$. More precisely, we need to scale them as

$$
\gamma=1+\epsilon / 2, \quad \ell=\epsilon \frac{\hat{\ell}}{2}, \quad \rho=\sqrt{\epsilon} v
$$

where $v$ is a new rescaled variable and $\hat{\ell}$ is fixed as $\epsilon \rightarrow 0$. Now, to leading order in $\epsilon$ we find that (C.4) becomes

$$
0=-\frac{\hat{\ell}^{2}}{4}-v_{0}^{2}+v_{0}^{4}, \quad \text { or } \quad v_{0}^{2}=\frac{1+\sqrt{1+\hat{\ell}^{2}}}{2}
$$

The integral for $\theta$, (C.6), becomes negligibly small away from $\rho \sim \rho_{0}$ since there a factor of $\ell$ multiplying. So it receives all its contribution from the small $\rho$ region, namely the finite $v$ region, see (C.9). We can write

$$
\begin{aligned}
\frac{\theta}{2} & =\frac{\hat{\ell}}{2} \int_{v_{0}}^{\infty} \frac{1}{v \sqrt{\tilde{D}}}, \quad \Longrightarrow \quad \hat{\ell}=\tan \theta \\
\tilde{D} & =\left(v^{2}-v_{0}^{2}\right)\left(v^{2}+v_{0}^{2}-1\right)
\end{aligned}
$$

We can similarly compute the integral for $s$,

$$
\begin{aligned}
\frac{s}{2} & =\int_{\rho_{0}}^{1} d \rho \frac{\rho}{\sqrt{1-\rho^{2}}}\left(\frac{1}{\sqrt{D}}-\frac{1}{\rho^{2}}\right)+\int_{\rho_{0}}^{1} d \rho \frac{1}{\rho \sqrt{1-\rho^{2}}} \\
& =\int_{v_{0}}^{\infty} d v v\left(\frac{1}{\sqrt{\tilde{D}}}-\frac{1}{v^{2}}\right)+\log 2-\log \rho_{0} \\
\frac{s}{2} & =\log 4-\frac{1}{2} \log \left(\frac{\epsilon}{\cos \theta}\right), \quad \Longrightarrow \frac{\epsilon}{\cos \theta}=16 e^{-s}
\end{aligned}
$$


where we used $\rho_{0}=\sqrt{\epsilon} v_{0}$ and the result (C.11), and the definition of $\tilde{D}$ in (C.12). Here we have split the integral in two terms, the first receives contributions only form the small $\rho$ region and the second, which can be done explicitly with no need to take the small $\rho_{0}$ limit (though we quoted here only the small $\rho_{0}$ answer).

We now want to compute $L$. We will compute instead

$$
\begin{gathered}
\frac{L}{4 g}-\frac{s}{2}=\frac{\epsilon}{2} \int_{\rho_{0}}^{1} d \rho \rho \sqrt{1-\rho^{2}}\left(\frac{1}{\sqrt{D}}-\frac{1}{\rho^{2}}\right)- \\
\int_{\rho_{0}}^{1} d \rho \frac{\rho^{3}}{\sqrt{1-\rho^{2}}}\left(\frac{1}{\sqrt{D}}-\frac{1}{\rho^{2}}+\epsilon \frac{\left(1-\rho^{2}\right)}{2 \rho^{4}}\right)-\int_{\rho_{0}}^{1} d \rho \frac{\rho}{\sqrt{1-\rho^{2}}} \\
\frac{L}{4 g}-\frac{s}{2}=\frac{\epsilon}{2} \int_{v_{0}}^{\infty} d v v\left(\frac{1}{\sqrt{\tilde{D}}}-\frac{1}{v^{2}}\right)- \\
\epsilon \int_{v_{0}}^{\infty} d v v^{3}\left(\frac{1}{\sqrt{\tilde{D}}}-\frac{1}{v^{2}}+\frac{1}{2 v^{4}}\right)-1+\frac{\rho_{0}^{2}}{2} \\
\frac{L}{4 g}-\frac{s}{2}=\epsilon\left(\frac{1}{4}-\frac{v_{0}^{2}}{2}\right)-1+\epsilon \frac{v_{0}^{2}}{2}=-1+\frac{\epsilon}{4}=-1+\cos \theta 4 e^{-s} \\
L-2 g s=-4 g+16 g \cos \theta e^{-\frac{L}{2 g}-2}
\end{gathered}
$$

Here we have split the integrals having in mind that we want an accuracy of order $\epsilon$. The first has an $\epsilon$ in front and we made sure that only the small $\rho$ region contributes. In the second we made sure that only the small $\rho$ region contributes up to order $\epsilon$. The last can can be evaluated exactly and we quoted here the small $\rho_{0}$ result.

Here we have in mind keeping $s$ fixed as we change $\theta$. Under these circumstances $\Delta$ stays fixed, since the $A d S_{2}$ part of the solution would always be the same. In addition, we know that for $\theta=0$ the result should vanish due to the BPS condition. Thus we find that

$$
\Delta-L=g(1-\cos \theta) \frac{16}{e^{2}} e^{-\frac{L}{2 g}}
$$

If we changed the angle in the AdS part, then instead of 1 in (C.22) we would get some function of $\phi$. However, since we know that for $\theta=\phi$ we should get zero due to the BPS condition, we conclude that for generic angles we get

$$
\Delta-L=g(\cos \phi-\cos \theta) \frac{16}{e^{2}} e^{-\frac{L}{2 g}}
$$

\section{C.1 Strong coupling expansion of the function $F$}

In order to compare this to the expected answer from the Luscher type correction we need to evaluate the function $F$ in (2.31) at strong coupling. This involves evaluating the function $\Phi$ in (2.8), (2.10) at $z^{[ \pm a]}$ at $q=0$. When $q=0$ we have that

$$
-1 / z^{[-a]}(0)=z^{[+a]}(0)=i\left(\sqrt{1+a^{2} /\left(16 g^{2}\right)}-a /(4 g)\right)=i\left(1+\frac{a}{4 g}+\cdots\right)
$$


which is very close to $i$, where the strong coupling expansion is tricky, since we cannot use (2.13). We need to compute

$$
\log F=i \Phi(y)-i \Phi(1 / y)=\frac{2}{\pi} \int_{0}^{\frac{\pi}{2}} d t \frac{\left(y^{4}-1\right)}{\left(1+y^{2}\right)^{2}-4 y^{2} \sin ^{2} t} \log \left[\frac{\sinh 4 \pi g \sin t}{4 \pi g \sin t}\right]
$$

with $y=z^{[a]}(0)$. Then the $y$ dependent factor can be well approximated by

$$
\left.\frac{\left(y^{4}-1\right)}{\left(1+y^{2}\right)^{2}-4 y^{2} \sin ^{2} t}\right|_{y=x^{[a]}(0)} \sim \frac{a}{4 g}\left[\frac{1}{\sin ^{2} t+\frac{a^{2}}{16 g^{2}}}\right]
$$

We now insert this into the integral (C.25), and split the integral into two pieces

$$
\begin{aligned}
\log F & =r_{1}+r_{2} \\
r_{1} & =\frac{a}{2 \pi g} \int_{0}^{\frac{\pi}{2}} d t\left[\frac{1}{\sin ^{2} t+\frac{a^{2}}{16 g^{2}}}\right] 4 \pi g \sin t=2 a \log \left[\frac{8 g}{a}\right]+o(1 / g) \\
r_{2} & =\frac{a}{2 \pi g} \int_{0}^{\frac{\pi}{2}} d t\left[\frac{1}{\sin ^{2} t+\frac{a^{2}}{16 g^{2}}}\right] \log \left[\frac{1-e^{-8 \pi g \sin t}}{8 \pi g \sin t}\right]= \\
r_{2} & =\int_{0}^{\infty} d t \frac{4 a}{v^{2}+4 a^{2} \pi^{2}} \log \left[\frac{1-e^{-v}}{v}\right]=2[a \log a-a-\log \Gamma(a+1)]
\end{aligned}
$$

where we have defined $t=v /(8 \pi g)$ in the integral for $r_{2}$ and taken the $g \rightarrow \infty$ limit. Summarizing, we get that the leading strong coupling approximation is

$$
F(a, g)=\frac{2^{6 a} g^{2 a}}{e^{2 a}(a !)^{2}}
$$

\section{Evaluating $t(q)$}

Let us now evaluate $t(q)$, given in (2.22). In order to perform the trace over the matrix indices it is convenient to write the reflection matrix in terms of the bulk $S$ matrix. The reason is that we will be able to use the bulk crossing equation to simplify the form of $t(q)$. We start by writing

$$
K^{A \dot{A}, B \dot{B}}(q)=\sigma_{b}\left(z^{+}, z^{-}\right) \frac{1+\frac{1}{\left(z^{+}\right)^{2}}}{1+\frac{1}{\left(z^{-}\right)^{2}}} \sqrt{\frac{z^{+}+1 / z^{+}}{z^{-}+1 / z^{-}}} S_{C D}^{A E}(-p, p) T_{E}^{\dot{A}} T_{F}^{\dot{B}} \mathcal{C}^{C B} \mathcal{C}^{D F},
$$

where $S$ is the full bulk $S$ matrix for one of the $\widetilde{s u}(2 \mid 2)$ factors, which obeys the crossing equation, with the identity in the right hand side. Here $p$ is the value of the momentum analytically continued so that we have $x^{ \pm}$in the mirror region. The square root arises because of a conventional way to define the bulk $S$ matrix. It is cancelling a square root in a the phase factor of the bulk $S$ matrix. The matrix $T$ converts the dotted indices into 
undotted indices. It arises in the precise implementation of the "unfolding" trick, where we replace a bulk magnon with momentum $p$ that transforms under $\widetilde{s u}(2 \mid 2)^{2}$ into two magnons of $\widetilde{s u}(2 \mid 2)_{D}$, one with momentum $p$ and the other with momentum $-p$, see appendix A. We then see that $\bar{K}$ in $(2.21)$ is essentially the same as $K$, but evaluated at $-\bar{p}$. More explicitly, we can write

$$
\bar{K}_{A \dot{A}, B \dot{B}}(q)=\sigma_{b}\left(-\frac{1}{z^{-}},-\frac{1}{z^{+}}\right) \frac{1+\left(z^{-}\right)^{2}}{1+\left(z^{+}\right)^{2}} \sqrt{\frac{z^{-}+1 / z^{-}}{z^{+}+1 / z^{+}}} S_{B M}^{G H}(\bar{p},-\bar{p}) T_{\dot{A}}^{N} T_{\dot{B}}^{M} \mathcal{C}_{G A} \mathcal{C}_{H N} .
$$

We can now insert this into the expression for $t(q)$. We will need to use that

$$
S_{C D}^{A E}(-p, p) \Sigma_{E}^{N} \mathcal{C}^{C B} \mathcal{C}^{D F} S_{B M}^{G H}(\bar{p},-\bar{p}) \Sigma_{F}^{M} \mathcal{C}_{G A} \mathcal{C}_{H N}=\operatorname{Tr}[\Sigma]^{2},
$$

here $\Sigma_{E}^{N}=T_{\dot{A}}^{N} T_{E}^{\dot{A}}$, where

$$
\Sigma=\operatorname{diag}(1,1,-1,-1)=(-1)^{F} .
$$

This arises because the action of the charge conjugation changes in the basis given by (A.4). The charge conjugation matrix can be taken to be

$$
\mathcal{C}_{A B}=\left(\begin{array}{cc}
-i \epsilon_{a b} & 0 \\
0 & \epsilon_{\alpha \beta}
\end{array}\right) \text {. }
$$

Equation (D.3) arises from the repeated use of the crossing equation. Ignoring charge conjugation matrices the identity we need is

$$
\mathcal{S}(-p, p) \mathcal{S}(\bar{p},-\bar{p})=\mathcal{S}(-p, p) \mathcal{S}(-\bar{p}, p) \mathcal{S}(p,-\bar{p}) \mathcal{S}(\bar{p},-\bar{p})=\mathbf{1}
$$

where $\mathcal{S}$ denotes the full $S$ matrix. When (D.3) is used, we get (2.24) in the main text. The series of operations we have done are most clearly summarized by the figure 9 . First we do the unfolding trick. Then the use of the crossing relation (D.6) amounts to moving the lines, as in figure 5, and untangling them. If we introduce the rotation matrix $m$, we can do all the same steps but we have insertions of the matrix $m$ or $m^{-1}$ along some lines. This is represented in figure 9 by the solid circles. Once we untangle the lines as in figure 5, we get get an insertion of $m$ on one line and an insertion of $m^{-1}$ on the other, leading to (2.25) (since the trace of $m$ or $m^{-1}$ are the same).

This gave us $t(q)$ for the fundamental mirror magnon. For the bound states we can use the fusion procedure. Given the form of $\sigma_{B}$ in (2.8), which involves a ratio of a function of $x^{+}$and $x^{-}$, then it is clear that the fusion procedure gives a $\sigma_{B}$ which is the same ratio, but evaluated at $z^{[+a]}$ and $z^{[-a]}$. The corresponding matrices are determined also by multiplying the matrices of the elementary constituents. All the manipulations we used above can be used again for these matrices. In particular, we can untangle the lines as in 9. The only new thing we need to understand is the set of states of a magnon bound state and the action of the matrix $m$. The matrix $m$ is

$$
\begin{gathered}
m_{a}=\operatorname{diag}(\overbrace{\left(e^{i \theta-i(a-1) \phi}, \cdots, e^{i \theta+i(a-1) \phi}\right.}^{a} \overbrace{e^{-i \theta-i(a-1) \phi}, \cdots, e^{-i \theta+i(a-1) \phi}}^{a}, \\
\overbrace{e^{-i a \phi}, \cdots, e^{i a \phi}}^{a+1}, \overbrace{e^{-i(a-2) \phi}, \cdots, e^{i(a-2) \phi}}^{a-1})
\end{gathered}
$$




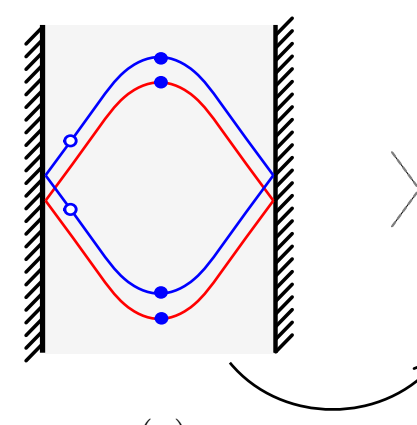

(a)

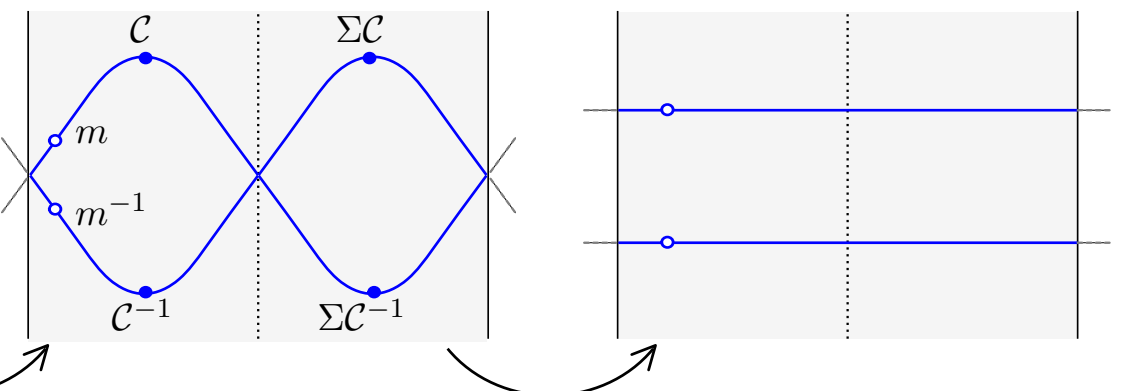

(b) (c)

Figure 9. (a) Original picture. White circles represent rotation matrices, one is $m$ and the other is $m^{-1}$. Solid circles represent charge conjugations. (b) Unfolded picture. In the unfolding of the dotted indices there is a change of basis that produces the $\Sigma$ 's. (c) Untangled picture. After using crossing we get two independent traces of the matrix $m$.

This can be understood as follows. The mirror magnon bound state arises from a $S L(2)$ sector fundamental magnons, giving rise to an $S L(2)$ representation of spin $2 j=a$, these lead to the components with the $a+1$ bracket. The other elements arise from acting with the supercharges in $\widetilde{s u}(2 \mid 2)$. Then we see that the trace gives the result quoted in (2.27). Thus, the cancelation of the bulk $S$ matrices, plus the form for $\sigma_{B}$ for the bound states, together with (D.7) lead to $t_{a}(q)$ in $(2.26)$.

\section{E Derivation of the BTBA equations}

In this appendix we derive the BTBA equations presented in the main text (4.2)-(4.9). We will do so in two different ways. In the first way, presented in section E.1, we will follow the original derivation of the TBA for the spectrum [37, 38] by embedding our open ABA equations into the closed ones. In the second way, presented in section E.2, we will take a more direct route and derive the BTBA from the thermodynamics in the mirror picture [17].

\section{E.1 Derivation by embedding into the bulk system}

The spectrum of closed strings or single trace operators in $\mathcal{N}=4 \mathrm{SYM}$ is governed by the so called Y-system [37]. The Y-system is a set of functions $Y_{a, s}(u)$ characterizing the ratio of the density of bulk excitation to the density of holes in the mirror channel [31]. The indices $(a, s)$ stands for rectangular representations of the bulk $S U(2,2 \mid 4)$ excitations and $u$ is the spectral parameter. The Y-function are subject to the general functional equation

$$
\frac{Y_{a, s}^{+} Y_{a, s}^{-}}{Y_{a+1, s} Y_{a-1, s}}=\frac{\left(1+Y_{a, s+1}\right)\left(1+Y_{a, s-1}\right)}{\left(1+Y_{a+1, s}\right)\left(1+Y_{a-1, s}\right)}
$$

where $f^{ \pm}=f(u \pm i / 2)$. For the bulk excitations, the Y's live in a fat hook bounded by $Y_{0, s}=\infty, Y_{2,|s|>2}=\infty$ and $Y_{a>2, \pm 2}=0$, (see fig 8.a). The Y-system is also equivalent to the Hirota equation for the T-functions as

$$
T_{a, s}^{+} T_{a, s}^{-}=T_{a, s+1} T_{a, s-1}+T_{a+1, s} T_{a-1, s}, \quad \text { where } \quad Y_{a, s}=\frac{T_{a, s+1} T_{a, s-1}}{T_{a+1, s} T_{a-1, s}}
$$


and enjoy a gauge invariance under $T_{a, s} \rightarrow g^{[ \pm a \pm s]} T_{a, s}$. For more details, see [37, 38]. The TBA equations for the $\mathrm{Y}$ functions are the solution to the functional relation (E.1), subject to the relevant boundary conditions and analytic behavior. The procedure of deriving the TBA equations in this fashion was carried out in [37, 38].

Operators on Wilson loops are associated to a string, or a spin chain, with boundaries. Suppose we start with such operators and go to the mirror picture where space and time are interchanged (see figure 2). In the mirror picture, one have exactly the same system of mirror particles as in the closed case. In the limit of large $T$, see figure 2 , these particles live on a large closed chain governed by the mirror asymptotic Bethe equations [35]. The boundaries in the original physical picture are mapped to two boundary states in the mirror past and future. These boundary states are determined by the boundary reflection matrix as we discussed in section 2. As opposed to the closed case where all mirror states are traced over in the partition function, in the overlap between boundary states only a subset of mirror excitations are summed over. Moreover, the weights of these excitations in the summation lead to a new asymptotic behavior for their densities. We therefore expect the the Y-system and the TBA equations to be identical to the ones in the closed case modulo projections and new sources.

In this section we will exploit that relation to derive the boundary TBA equations. That is, we will first map the open $\widetilde{s u}(2 \mid 2)_{\mathrm{D}}$ ABA equations (3.9)-(3.11) into a folded version of the standard closed $\widetilde{s u}(2 \mid 2)^{2}$ ones. That map is nothing but the embedding of the diagonal $\widetilde{s u}(2 \mid 2)_{\mathrm{D}}$ excitations in the full $\widetilde{s u}(2 \mid 2)_{\mathrm{L}} \times \widetilde{s u}(2 \mid 2)_{\mathrm{R}}$ by restricting to singlet excitations of the diagonal $\widetilde{s u}(2 \mid 2)_{\mathrm{D}}$ preserved by the boundary. Having done so, the corresponding BTBA equations will follow from the derivation of the closed TBA ones [38]. We will only add the angles that enter as (diagonal) twists and the boundary dressing phase that enters as a momentum dependent chemical potential for the momentum carrying excitations. As most of the details are the same as in the closed case, we will be brief.

As we discussed in section 2 , and in appendix A, the diagonal $\widetilde{s u}(2 \mid 2)_{\mathrm{D}}$ magnon excitations transforms in a tensor representation of the bulk $\widetilde{s u}(2 \mid 2)_{\mathrm{L}} \times \widetilde{s u}(2 \mid 2)_{\mathrm{R}}$. Correspondingly, the $\mathrm{ABA}$ equations can be embedded into the closed ones together with the addition of the 
reflection matrix. That is, (3.9)-(3.11) can also be written as in a redundant way as

$$
\begin{aligned}
e^{i \phi-i \theta} & =\left.\frac{\mathbb{B}^{(-)} Q_{2}^{+}}{\mathbb{B}^{(+)} Q_{2}^{-}}\right|_{u_{1, k}} \\
-e^{2 i \theta} & =\left.\frac{Q_{1}^{+} Q_{2}^{--} Q_{3}^{+}}{Q_{1}^{-} Q_{2}^{++} Q_{3}^{-}}\right|_{u_{2, k}} \\
e^{i \phi-i \theta} & =\left.\frac{\mathbb{R}^{(-)} Q_{2}^{+}}{\mathbb{R}^{(+)} Q_{2}^{-}}\right|_{u_{3, k}} \\
R_{0}^{-2}(u) & =\left[\left(\frac{x^{+}}{x^{-}}\right)^{L} S(u)^{2} \frac{R_{3}^{-} B_{1}^{-}}{R_{3}^{+} B_{1}^{+}}\right] \times\left.\left[\left(\frac{x^{+}}{x^{-}}\right)^{L} \bar{S}(u)^{2} \frac{\bar{R}_{3}^{-} \bar{B}_{1}^{-}}{\bar{R}_{3}^{+} \bar{B}_{1}^{+}}\right]\right|_{u_{4, k}} \\
e^{-i \phi+i \theta} & =\left.\frac{\mathbb{R}^{(-)} \bar{Q}_{2}^{+}}{\mathbb{R}^{(+)} \bar{Q}_{2}^{-}}\right|_{-u_{3, k}} \\
-e^{-2 i \theta} & =\left.\frac{\bar{Q}_{1}^{+} \bar{Q}_{2}^{--} \bar{Q}_{3}^{+}}{\bar{Q}_{1}^{-} \bar{Q}_{2}^{++} \bar{Q}_{3}^{-}}\right|_{-u_{2, k}} \\
e^{-i \phi+i \theta} & =\left.\frac{\mathbb{B}^{(-)} \bar{Q}_{2}^{+}}{\mathbb{B}^{(+)} \bar{Q}_{2}^{-}}\right|_{-u_{1, k}}
\end{aligned}
$$

where

$$
\begin{aligned}
R_{l}(u) & =\prod_{j=1}^{K_{l}} \frac{x(u)-x_{l, j}}{\left(x_{l, j}\right)^{1 / 2}}, & B_{l}(u)=\prod_{j=1}^{K_{l}} \frac{\frac{1}{x(u)}-x_{l, j}}{\left(x_{l, j}\right)^{1 / 2}}, & Q_{l}(u)=\prod_{j=1}^{K_{l}}\left(u-u_{l, j}\right) \\
\bar{R}_{l}(u) & =\prod_{j=1}^{K_{l}} \frac{x(u)+x_{l, j}}{\left(x_{l, j}\right)^{1 / 2}}, & \bar{B}_{l}(u)=\prod_{j=1}^{K_{l}} \frac{\frac{1}{x(u)}+x_{l, j}}{\left(x_{l, j}\right)^{1 / 2}}, & \bar{Q}_{l}(u)=\prod_{j=1}^{K_{l}}\left(u+u_{l, j}\right) \\
\mathbb{R}^{( \pm)}(u) & =\prod_{j=1}^{K_{4}} \frac{\left(x(u)-x_{j}^{\mp}\right)\left(x(u)+x_{j}^{ \pm}\right)}{\left(x_{j}^{+} x_{j}^{-}\right)^{1 / 2}}, & \mathbb{B}^{( \pm)}(u) & =\prod_{j=1}^{K_{4}} \frac{\left(\frac{1}{x(u)}-x_{j}^{\mp}\right)\left(\frac{1}{x(u)}+x_{j}^{ \pm}\right)}{\left(x_{j}^{+} x_{j}^{-}\right)^{1 / 2}} \\
S(u) & =\prod_{j \neq k}^{K_{4}} S_{0}\left(p(u), p_{j}\right)^{2}, & \bar{S}(u) & =\prod_{j \neq k}^{K_{4}} S_{0}\left(p(u),-p_{j}\right)^{2}
\end{aligned}
$$

where we used that $S_{0}\left(p_{j},-p(u)\right)=S_{0}\left(p(u),-p_{j}\right)$.

Note that equations for nodes 1-3 in (E.3) are equivalent to the ones in nodes 57. ${ }^{11}$ However, here we will think about these as describing the left and right parts of the bulk magnon excitations correspondingly. ${ }^{12}$ We see that momentum carrying excitations

\footnotetext{
${ }^{11}$ Remember that in physical kinematics, where (E.3) is written, $\left|x^{[a]}\right|>1$ and therefore $x^{ \pm}(-u)=$ $-x^{\mp}(u), x(-u)=-x(u), p(-u)=-p(u)$ and $\epsilon(-u)=\epsilon(u)$.

${ }^{12}$ After rearrangement of the quantum numbers discussed in the appendix.
} 
come in pairs with opposite momenta. The nested level excitations also comes in pairs of rapidities $u_{5}=-u_{3}, u_{6}=-u_{2}$ and $u_{7}=-u_{1}$. That is, $\bar{Q}_{l}(u)$ is nothing but $Q_{8-l}(u)$ with roots $u_{l, j}=-u_{8-l, j}$.

We will now use that embedding to derive from them the asymptotic values of the left and right T-functions. Of course, these are not independent and we only have one set of independent $\widetilde{s u}(2 \mid 2)_{\mathrm{D}}$ T-functions.

In the asymptotic $L \rightarrow \infty$ limit the right $\left(T_{a, s \geq 0}\right)$ and left $\left(T_{a, s \leq 0}\right)$ T systems decouples. ${ }^{13}$ The asymptotic values of $T_{1,1}$ and $T_{1,-1}$, whose analyticity would lead to equations in nodes 1-3 and 5-7 in (E.3) correspondingly, are derived as in the periodic case [37]. In what follows, we will use bold face $(\mathbf{Y}, \mathbf{T})$ for the asymptotic values of Y's and T's. Up to a gauge transformation, the asymptotic $\mathbf{T}_{1,1}^{\mathrm{R}}$ and $\mathbf{T}_{1,-1}^{\mathrm{L}}$ are

$$
\begin{aligned}
\mathbf{T}_{1,1}^{\mathrm{R}} & =\frac{\mathbb{R}^{-(+)}}{\mathbb{R}^{-(-)}}\left(e^{-i \theta} \frac{Q_{2}^{--} Q_{3}^{+}}{Q_{2} Q_{3}^{-}}-e^{-i \phi} \frac{\mathbb{R}^{-(-)} Q_{3}^{+}}{\mathbb{R}^{-(+)} Q_{3}^{-}}+e^{i \theta} \frac{Q_{2}^{++} Q_{1}^{-}}{Q_{2} Q_{1}^{+}}-e^{i \phi} \frac{\mathbb{B}^{+(+)} Q_{1}^{-}}{\mathbb{B}^{+(-)} Q_{1}^{+}}\right) \\
\mathbf{T}_{1,-1}^{\mathrm{L}} & =\frac{\mathbb{R}^{-(+)}}{\mathbb{R}^{-(-)}}\left(e^{i \theta} \frac{\bar{Q}_{2}^{--} \bar{Q}_{3}^{+}}{\bar{Q}_{2} \bar{Q}_{3}^{-}}-e^{i \phi} \frac{\mathbb{R}^{-(-)} \bar{Q}_{3}^{+}}{\mathbb{R}^{-(+)} \bar{Q}_{3}^{-}}+e^{-i \theta} \frac{\bar{Q}_{2}^{++} \bar{Q}_{1}^{-}}{\bar{Q}_{2} \bar{Q}_{1}^{+}}-e^{-i \phi} \frac{\mathbb{B}^{+(+)} \bar{Q}_{1}^{-}}{\mathbb{B}^{+(-)} \bar{Q}_{1}^{+}}\right)
\end{aligned}
$$

The right 1-3 in (E.3) ABA equations are obtained by demanding analyticity of $\mathbf{T}_{1,1}^{\mathrm{R}}$ when $u$ goes to $u_{1, k}-\frac{i}{2}, u_{2, k}$ and $u_{3, k}+\frac{i}{2}$. The equivalent right equations 5-7 in (E.3) are obtained from the analyticity of $\mathbf{T}_{1,-1}^{\mathrm{L}}$ when $u$ goes to $-u_{1, k}-\frac{i}{2},-u_{2, k}$ and $-u_{3, k}+\frac{i}{2}$. In the gauge where $\mathbf{T}_{0, s}^{\mathrm{L} / \mathrm{R}}=\mathbf{T}_{a, 0}^{\mathrm{L} / \mathrm{R}}=1$, the other $\mathrm{T}$ functions of the right and left (decoupled) $S U(2 \mid 2)$ wings are obtained from the generating functional $[37,55-57]$

$$
\begin{aligned}
\mathcal{W}= & {\left[1-e^{i \phi} \frac{\mathbb{B}^{+(+)} Q_{1}^{-}}{\mathbb{B}^{+(-)} Q_{1}^{+}} \frac{\mathbb{R}^{-(+)}}{\mathbb{R}^{-(-)}} D\right]\left[1-e^{i \theta} \frac{Q_{2}^{++} Q_{1}^{-}}{Q_{2} Q_{1}^{+}} \frac{\mathbb{R}^{-(+)}}{\mathbb{R}^{-(-)}} D\right]^{-1} } \\
& \times\left[1-e^{-i \theta} \frac{Q_{2}^{--} Q_{3}^{+}}{Q_{2} Q_{3}^{-}} \frac{\mathbb{R}^{-(+)}}{\mathbb{R}^{-(-)}} D\right]^{-1}\left[1-e^{-i \phi} \frac{Q_{3}^{+}}{Q_{3}^{-}} D\right]
\end{aligned}
$$

as

$$
\mathcal{W}=\sum_{s=0}^{\infty} \mathbf{T}_{1, s}^{[1-s]} D^{s}, \quad \mathcal{W}^{-1}=\sum_{a=0}^{\infty}(-1)^{a} \mathbf{T}_{a, 1}^{[1-a]} D^{a}, \quad D=e^{-i \partial_{u}}
$$

Similarly, for negative $s$ we reverse the sign of the angles and use $\bar{Q}$ instead of $Q$. Their analyticity leads to the Bethe equations for the bound states. In the vacuum, the T's are independent of $u$. Their constant values read

$$
\begin{aligned}
& \mathbf{T}_{a, 1}^{\mathrm{R}}=\mathbf{T}_{a,-1}^{\mathrm{L}}=(-1)^{a} 2(\cos \phi-\cos \theta) \frac{\sin a \phi}{\sin \phi}, \\
& \mathbf{T}_{1, s}^{\mathrm{R}}=\mathbf{T}_{1,-s}^{\mathrm{L}}=\quad-2(\cos \phi-\cos \theta) \frac{\sin s \theta}{\sin \theta} .
\end{aligned}
$$

\footnotetext{
${ }^{13}$ Throughout this section the word "asymptotic", means this large $L$ limit, which should not be confused with the large $u$ limit.
} 
Using the Hirota equation and the definition of the Y-functions in the right and left decoupled wings (E.2) we read the corresponding values of the asymptotic Y's

$$
\begin{aligned}
\mathbf{Y}_{1,1}=-\frac{\cos \theta}{\cos \phi}, & \mathbf{Y}_{1, s}=\frac{\sin [(s+1) \theta] \sin [(s-1) \theta]}{\sin ^{2} \theta} \\
\mathbf{Y}_{2,2}=-\frac{\cos \phi}{\cos \theta}, & \mathbf{Y}_{a, 1}=\frac{\sin ^{2} \phi}{\sin [(a+1) \phi] \sin [(a-1) \phi]}
\end{aligned}
$$

For the asymptotic $Y_{a, 0}\left(\mathbf{Y}_{a, 0}\right)$ we have

$$
\mathbf{Y}_{a, 0}=\left(\frac{x^{[-a]}}{x^{[+a]}}\right)^{2 L} \frac{\phi^{[-a]}}{\phi^{[+a]}} \mathbf{T}_{a,-1}^{L} \mathbf{T}_{a, 1}^{R}
$$

Here, $\left(\frac{x^{[-a]}}{x^{[+a]}}\right)^{2 L} \frac{\phi^{[-a]}}{\phi^{[+a]}}$ is a zero mode of the discrete Laplace equation $\frac{\mathcal{A}_{a}^{+} \mathcal{A}_{a}^{-}}{\mathcal{A}_{a+1} \mathcal{A}_{a-1}}=1$ [37]. It comes about because $\mathbf{T}^{\mathrm{R}}$ and $\mathbf{T}^{\mathrm{L}}$ in (E.5)-(E.8) are written in different gauges. It is determined by demanding that $Y_{1,0}\left(u_{4, k}\right)=-1$ gives the $4^{\text {th }}$ node Bethe equation. We find

$$
\frac{\phi^{-}}{\phi^{+}}=R_{0}^{2}(u) S^{2}(u) \bar{S}^{2}(u) \frac{\mathbb{R}^{-(-)} \mathbb{B}^{+(+)}}{\mathbb{R}^{+(+)} \mathbb{B}^{-(-)}} \frac{B_{1}^{+} \bar{B}_{1}^{+} B_{3}^{-} \bar{B}_{3}^{-}}{B_{1}^{-} \bar{B}_{1}^{-} B_{3}^{+} \bar{B}_{3}^{+}}
$$

The boundary crossing equation (2.7) is then obtained by demanding that $\mathbf{Y}_{1,1}$ is invariant under crossing (provided that the bulk dressing phase obeys the bulk crossing equation). In particular, in the vacuum we get

$$
\mathbf{Y}_{a, 0}=4 \frac{e^{i \chi\left(z^{[+a]}\right)+i \chi\left(1 / z^{[-a]}\right)}}{e^{i \chi\left(z^{[-a]}\right)+i \chi\left(1 / z^{[+a]}\right)}}\left(\frac{z^{[-a]}}{z^{[+a]}}\right)^{2 L+2}(\cos \phi-\cos \theta)^{2} \frac{\sin ^{2} a \phi}{\sin ^{2} \phi}
$$

Finally, note that $\mathbf{T}_{a,-s}^{\mathrm{L}}(u)$ can be obtained from $\mathbf{T}_{a, s}^{\mathrm{R}}(u)$ in two steps. First reflect the sign of $u$ by considering $\mathbf{T}_{1,1}(-u)$. Second, flip the signs of all the shifts and the angles. In mirror kinematics, that second step that basically amount to a complex conjugation, is equivalent to a gauge transformation [58]. We therefore find that in mirror kinematics $\mathbf{T}_{a,-s}^{\operatorname{mir}}(u) \simeq \mathbf{T}_{a, s}^{\operatorname{mir}}(-u)$ and as a result $\mathbf{Y}_{a,-s}^{\operatorname{mir}}(u)=\mathbf{Y}_{a, s}^{\operatorname{mir}}(u) \cdot{ }^{14}$ As the closed TBA equation respects that symmetry, we expect all solutions to the BTBA equations to respect it. That is, we have a folded version of the bulk Y-system where $Y_{a,-s}(u)$ is identified with $Y_{a, s}(-u)$ as presented in the main text (4.1). Such a folding is also expected a priory as the Wilson lines break the bulk $S U(2,2 \mid 4)$ symmetry (at $\theta=\phi=0$ ) down to $O S p\left(4^{*} \mid 4\right)$. For the ground state, we expect all functions to be symmetric.

We conclude that the BTBA equations, once divided by the asymptotic ones, are the same as in the closed case. The only two differences are the modified asymptotic solutions (E.11), (E.14) and the folding $Y_{a,-s}(u)=Y_{a, s}(-u)$. These are the BTBA equations (4.2)-(4.9) presented in the main text.

\footnotetext{
${ }^{14}$ To see that $\mathbf{Y}_{a, 0}^{\mathrm{mir}}$ is a symmetric function note that in mirror kinematics, $x^{[a]}(-u)=-1 / x^{[-a]}$ and that $\chi(-x)=\chi(x)$.
} 


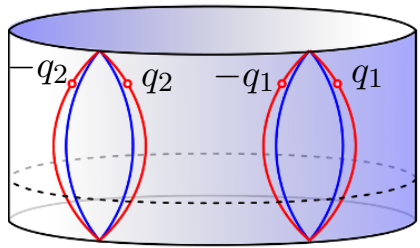

(a)
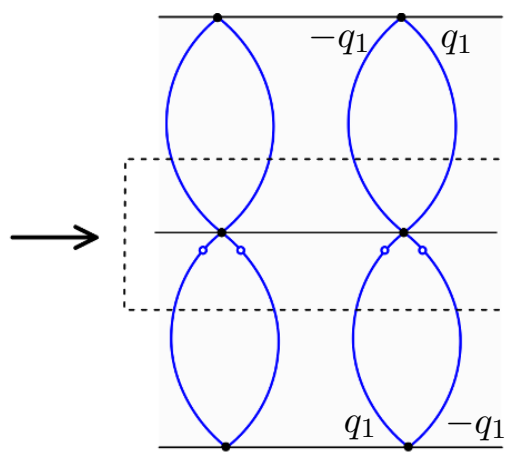

(b)

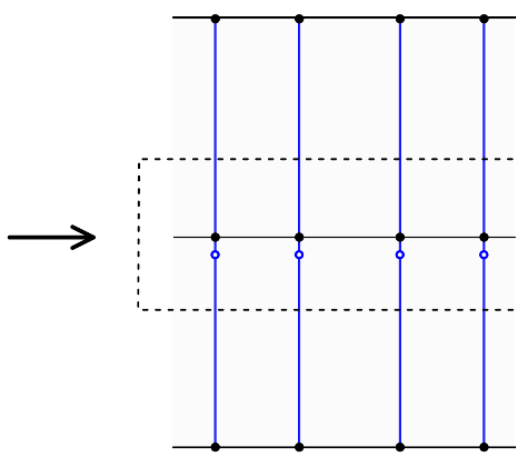

(c)

Figure 10. (a) Original computation. The boundary states create and annihilate pairs of particles. The blue and red lines represent the two $\widetilde{s u}(2 \mid 2)$ representations of each bulk magnon. The doted line represents the projection on to states that obey the Bethe equations for a chain that is closed along the horizontal direction. The red dots are the rotation matrices $m$ in (2.15) and the black dots are the boundary dressing phase. (b) The same in the unfolded picture. We have a single $\widetilde{s u}(2 \mid 2)$ group. We continue to have insertions of the matrix $m$ and the dressing phase. The Bethe equations now involve some operation which also involves the bottom part. (c) The same in the untangled picture. Here for each line we take the trace over all four states of the magnon.

\section{E.2 Direct derivation of the boundary TBA}

The derivation of boundary TBA equations from the boundary state (2.18) is fairly standard in relativistic theories $[17,18]$. Here we need to follow the same steps.

After doing the flip between space and time, see figure 2, we have a past and a future boundary characterized by the matrices $K$ and $\bar{K}$ which give the probability amplitude for creating or annihilating a pair of particles (2.18), (2.20). This pair has opposite momenta and it is in the singlet representation of an $S U(2 \mid 2)_{D}$, since this is a symmetry preserved by the boundary state. By independent creation events we can create a multiparticle state. We can graphically represent the quantity we want to evaluate as in figure 10(a). Along the spatial direction we have a closed circle of length $T$, we need to solve the Asymptotic Bethe equations on this circle and find the Bethe eigenstates. Only the subset of momenta that corresponds to Bethe eigenstates can propagate. These states propagate for Euclidean time $L$. These asymptotic Bethe equations are the ones in the mirror theory and were written in [32], following [20]. These equations involve various roots, $u_{1}, \cdots u_{7}$, where $u_{4}$ are the momentum carrying roots. The only new feature is that we are considering states which are composed of pairs of particles with opposite momenta. This imposes the condition that the $u_{4}$ roots should appear in pairs. In other words a root $u_{4}$ should appear together with a root $-u_{4}$. The boundary state is invariant under a diagonal $S U(2 \mid 2)_{D}$. The condition that we only have singlets under $S U(2 \mid 2)_{D}$ implies that if roots $u_{a}$, with $a=1,2,3$ appear, then so should roots $u_{8-a}=-u_{a}$. This will be more clearly seen below.

For this multiparticle state we can perform an unfolding and untangling trick identical to the one we did for a single particle in section 2, see figure 10(b),(c). Now we have particles for a single $\widetilde{s u}(2 \mid 2)$ chain, but with insertions associated to the rotation matrix $m$ as well as the dressing phase, $\sigma_{B}(q)$. We can view these as "chemical" potentials in 


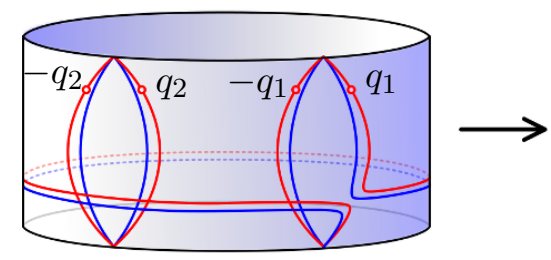

(a)

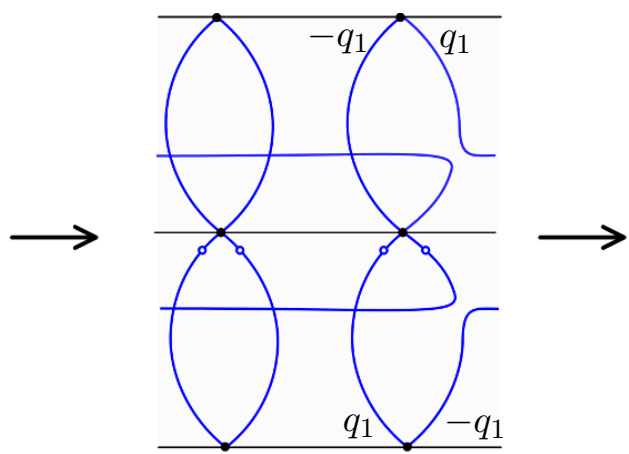

(b)

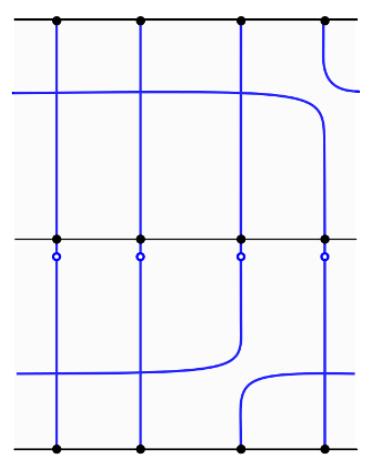

(c)

Figure 11. The Bethe equations in the various pictures come from demanding that each full particle can be taken around the chain producing a total phase of 1. (a) The Bethe equation in the original picture. (b) The Bethe equation in the unfolded picture comes from taking both a top particle with momentum $q$ and a bottom particle with momentum $-q$ around the chain. (c) The same but in the untangled picture.

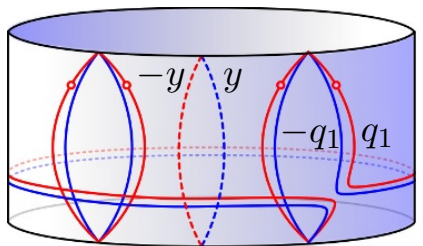

(a)

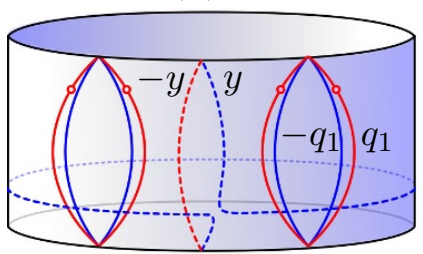

(b)

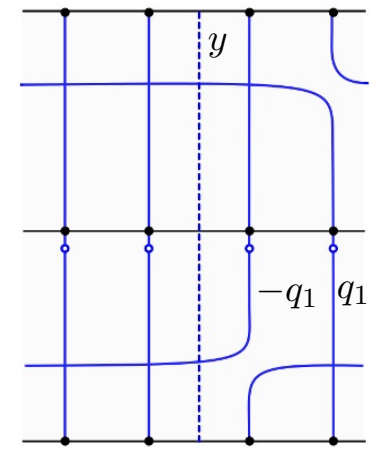

(c)

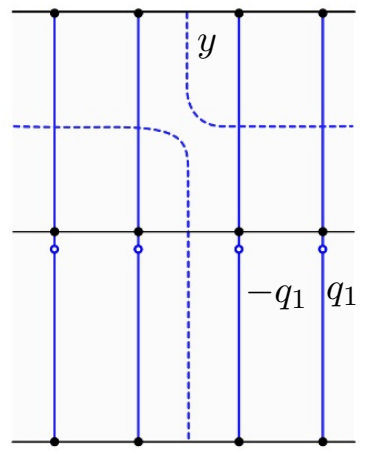

$(\mathbf{d})$

Figure 12. (a) Bethe equations for the momentum carrying nodes in the original picture. The doted lines represent the various nested levels, with red and blue for each of the $\widetilde{s u}(2 \mid 2)$ factors. Here $y$ represents the rapidity of levels $u_{1}, u_{2}$ or $u_{3}$, and $-y$ is the rapidity of the $u_{5}, u_{6}$ or $u_{7}$ levels. (b) Bethe equation for the nesting levels. (c) Equations for the momentum carrying nodes in the unfolded and untangled picture. Note that both the particle with momentum $q$ and the one with momentum $-q$ cross the nesting doted line. (d) Bethe equation for the nested line.

a thermodynamic computation. The Bethe equations in this new picture look slightly more complicated because taking a single full particle around the circle, as in figure 11(a), amounts to taking a pair of particles around the circle, as in figure 11(b),(c). We should project onto states that obey these equations. These equations are for the full matrices. We can do the nesting procedure and follow the unfolding for the various levels of the nesting. The Bethe equations for the various nesting levels can be graphically represented as in figure 12. The final result is that the equations are identical to the ones we would get in a situation where we have the full mirror theory, but we impose that each root $u_{a}$ is 
accompanied by a root $-u_{8-a}$.

The final Bethe equations in the mirror picture are

$$
\begin{aligned}
1 & =e^{i p\left(u_{4}^{j}\right) T} \prod_{k} S_{44}\left(u_{4}^{j}, u_{4}^{k}\right) S_{44}\left(u_{4}^{j},-u_{4}^{k}\right) \prod_{l} S_{43}\left(u_{4}^{j}, u_{3}^{l}\right) S_{43}\left(-u_{4}^{j}, u_{3}^{l}\right) \\
1 & =\prod_{k} S_{34}\left(u_{3}^{l}, u_{4}^{k}\right) S_{34}\left(u_{3}^{l},-u_{4}^{k}\right) \prod_{m} S_{32}\left(u_{3}^{l}, u_{2}^{m}\right) \\
-1 & =\prod_{m} S_{32}\left(u_{3}^{m}, u_{2}^{l}\right) \prod_{n} S_{33}\left(u_{3}^{l}, u_{3}^{n}\right)
\end{aligned}
$$

where $S_{a b}$ are the $S$ matrices (really just phases) between the impurities of the various kinds. We have removed the $u_{1}$ roots by turning them into $u_{3}$ roots, just to make the equations more compact. The $S_{a b}$ are the same as the one that appear in the ordinary mirror Bethe equations in [32], see also [20]. In this form, these equations follow in a straightforward fashion from figure $12(\mathrm{c})(\mathrm{d})$. Note, in particular, that the last factor in (E.15) can also be written as $S_{43}\left(-u_{4}^{j}, u_{3}^{l}\right)=S_{45}\left(u_{4}^{j}, u_{5}^{l}\right)$ with $u_{5}^{l}=-u_{3}^{l}$. So that we can view the whole set of equations, (E.15)-(E.17), as arising from the full chain, with both $\widetilde{s u}(2 \mid 2)$ factors, but in a configuration with the roots related by $u_{8-a}=-u_{a}$, as we discussed above.

We can simply add the rotation matrices to this picture. They act diagonally on the various impurities of the various levels. They appear as chemical potentials. The potentials are as follows. The momentum carrying modes, labeled by $u_{4}$, are in the $S L(2)$ sector and have spacetime charges, so we get a factor of $e^{-2 i \phi}$, one $e^{-i \phi}$ for the particle of momentum $q$ and one for the one with momentum $-q$. The next root, $u_{3}$ or $u_{1}$, is the action of a fermionic generator that changes a fermion into a boson, so that we need to add $e^{i \theta+i \phi}$. Then a bosonic generator with $e^{-2 i \theta}$ is associated to the root $u_{2}$. Taking into account the number of roots of each type in a magnon bound state, we can get the chemical potential coming from the angles for each magnon bound state [32]. In addition, the boundary dressing phase also appears as a kind of chemical potential. For each pair of momenta $q$ and $-q$ we have a factor of

$$
\sigma_{B} \bar{\sigma}_{B} \equiv \sigma_{B}\left(z^{+}, z^{-}\right) \sigma_{B}\left(-\frac{1}{z^{-}},-\frac{1}{z^{+}}\right)
$$

The derivation of the TBA equations for this system follows the same essential steps as the derivation for the full TBA for the closed string. There are only minor differences due to the constraints on the position of the Bethe roots. For example, for the roots $u_{4}$ we only need to know the density in the region $u_{4}>0$, since there is always another associated root at $-u_{4}$. Thus, all integrals over $u_{4}$ will run over $u_{4}>0$. We can define the $Y$ functions, as usual, in terms of densities of particles over densities of holes. We then run through the usual argument [33], but keeping track of the range of integration, etc. We end up with the 
boundary TBA equations

$$
\begin{aligned}
& \log Y_{1,1}=i \theta+i \phi+K_{m-1} * \log \frac{1+\bar{Y}_{1, m}}{1+Y_{m, 1}}+\mathcal{R}_{1 a}^{(01)} * \log \left(1+Y_{a, 0}\right) \\
& \log \bar{Y}_{2,2}=i \theta+i \phi+K_{m-1} * \log \frac{1+\bar{Y}_{1, m}}{1+Y_{m, 1}}+\mathcal{B}_{1 a}^{(01)} * \log \left(1+Y_{a, 0}\right) \\
& \log \bar{Y}_{1, s}=2 i(s-1) \theta-K_{s-1, t-1} * \log \left(1+\bar{Y}_{1, t}\right)-K_{s-1} * \log \frac{1+Y_{1,1}}{1+\bar{Y}_{2,2}} \\
& \log Y_{a, 1}=i 2(a-1) \phi-K_{a-1, b-1} * \log \left(1+Y_{b, 1}\right)-K_{a-1} * \log \frac{1+Y_{1,1}}{1+\bar{Y}_{2,2}}+ \\
& +\left[\mathcal{R}_{a b}^{(01)}+\mathcal{B}_{a-2, b}^{(01)}\right] * \log \left(1+Y_{b, 0}\right) \\
& +\left[2 \mathcal{S}_{a b}-\mathcal{R}_{a b}^{(11)}+\mathcal{B}_{a b}^{(11)}\right] * \log \left(1+Y_{b, 0}\right)+2\left[\mathcal{R}_{a b}^{(10)}+\mathcal{B}_{a, b-2}^{(10)}\right] \text { s }_{\text {ym }}^{*} \log \left(1+Y_{b, 1}\right)+ \\
& +2 \mathcal{R}_{a 1}^{(10)} \underset{\text { sym }}{*} \log \left(1+Y_{1,1}\right)-2 \mathcal{B}_{a 1}^{(10)} \underset{\text { sym }}{*} \log \left(1+\bar{Y}_{2,2}\right)
\end{aligned}
$$

where the kernels are listed in appendix E.3 and are the same as in [37, 38] but with $\bar{Y}_{a, s}^{\text {(here) }}=1 / Y_{a, s}^{\text {(there) }}$. The values of the angle dependent form of the chemical potential for each $Y_{a, s}$ function follows from the particle content of each mirror bound state associated to each $Y_{a, s}$ function [32]. If we set to zero the $Y_{0, a}$ that appear in the convolutions, we get the large $L$ solution quoted in (4.7), (4.9). These equations look similar to the ones in $[59,60]$ for twisted boundary conditions, apart from the folding symmetry and the boundary dressing function $\sigma_{B}$.

Finally, the expression for the energy is the one quoted in (4.11).

\section{E.3 Kernels conventions}

For the kernels we use the same definitions as in [37, 38],

$$
\begin{aligned}
K_{n}(u, v) & =\frac{2 n / \pi}{n^{2}+4(u-v)^{2}}, \quad K_{n, m}(u, v)=\sum_{j=-\frac{n-1}{2}}^{\frac{n-1}{2}} \sum_{k=-\frac{m-1}{2}}^{\frac{m-1}{2}} K_{2 j+2 k+2}(u, v), \\
\mathcal{R}_{n m}^{(a b)}(u, v) & =\sum_{j=-\frac{n-1}{2}}^{\frac{n-1}{2}} \sum_{k=-\frac{m-1}{2}}^{\frac{m-1}{2}} \frac{1}{2 \pi i} \frac{d}{d v} \log \frac{r(u+i a / 2+i j, v-i b / 2+i k)}{r(u-i a / 2+i j, v+i b / 2+i k)} \\
\mathcal{B}_{n m}^{(a b)}(u, v) & =\sum_{j=-\frac{n-1}{2}}^{\frac{n-1}{2}} \sum_{k=-\frac{m-1}{2}}^{\frac{m-1}{2}} \frac{1}{2 \pi i} \frac{d}{d v} \log \frac{b(u+i a / 2+i j, v-i b / 2+i k)}{b(u-i a / 2+i j, v+i b / 2+i k)} \\
\mathcal{S}_{n m}(u, v) & =\frac{1}{2 \pi i} \frac{d}{d v} \log \sigma\left(x^{[ \pm n]}(u), x^{[ \pm m]}(v)\right) \\
r(u, v) & =\frac{x(u)-x(v)}{\sqrt{x(v)}}, \quad b(u, v)=\frac{1 / x(u)-x(v)}{\sqrt{x(v)}}
\end{aligned}
$$

Fourier transformations of $K_{n}(u, 0)$ and $K_{n, m}(u, 0)$ are used at different stages,

$$
\widetilde{K}_{n}(w)=\operatorname{sign}(n) e^{-|n w| / 2}, \quad \widetilde{K}_{m, n}(w)=\operatorname{coth} \frac{|w|}{2}\left[e^{-\frac{|w|}{2}|m-n|}-e^{-\frac{|w|}{2}(m+n)}\right]-\delta_{m, n} .
$$




\section{F Perturbative solution of the small $\phi$ TBA}

Before we start with the perturbative expansion of the small $\phi$ TBA system, let us see in more detail how the simplified system (5.15)-(5.20) arises from (5.6)-(5.12). In order to simplify (5.6), (5.7) we have to convolute their $K_{m-1}$ terms with $s * s^{-1}$ and use the identity

$$
s^{-1} * K_{m-1}=K_{1, m-1}+\delta_{2, m}, \quad \text { for } s(u)=\frac{1}{2 \cosh (\pi u)} .
$$

We then use the TBA equation for $\mathcal{X}_{2}$ and the exact relations

$$
\begin{aligned}
& \mathcal{R}_{1 a}^{(01)}(u, v)+\mathcal{B}_{1 a}^{(01)}(u, v)=K_{a}(u, v), \\
& \mathcal{R}_{1 a}^{(01)}(u, v)-\mathcal{B}_{1 a}^{(01)}(u, v)=\hat{K}_{y, a}(u, v)=K\left(u, v-i \frac{a}{2}\right)-K\left(u, v+i \frac{a}{2}\right),
\end{aligned}
$$

where

$$
K(u, v)=\frac{1}{2 \pi i} \sqrt{\frac{4 g^{2}-u^{2}}{4 g^{2}-v^{2}}} \frac{1}{v-u} .
$$

Regarding the other TBA equations, we can simplify them by convoluting with

$$
\left(K_{l-1, m-1}+\delta(u) \delta_{l, m}\right)^{-1}=\delta(u) \delta_{l, m}-s I_{l, m}, \quad I_{l, m}=\delta_{l+1, m}+\delta_{l-1, m}
$$

The simplified TBA equations (5.15)-(5.20) are useful to solve for the Y-functions in the small $\phi$-limit perturbatively. We should begin by expanding the $\mathrm{Y}$-functions in powers of $g^{2}, 15$

$$
\begin{aligned}
\Psi & =\Psi^{(0)}+\Psi^{(1)} g^{2}+\Psi^{(2)} g^{4}+\cdots \\
\Phi & =\Phi^{(0)}+\Phi^{(1)} g^{2}+\Phi^{(2)} g^{4}+\cdots \\
\mathcal{Y}_{m} & =\mathcal{Y}_{m}^{(0)}\left(1+\mathcal{Y}_{m}^{(1)} g^{2}+\mathcal{Y}_{m}^{(2)} g^{4}+\cdots\right) \\
\mathcal{X}_{m} & =\mathcal{X}_{m}^{(0)}+\mathcal{X}_{m}^{(1)} g^{2}+\mathcal{X}_{m}^{(2)} g^{4}+\cdots \\
\mathbb{C}_{a} & =\mathbb{C}_{a}^{(2)} g^{2}+\mathbb{C}_{a}^{(2)} g^{4}+\mathbb{C}_{a}^{(3)} g^{6}+\cdots
\end{aligned}
$$

The leading orders in the expansion (F.6) can be obtained by setting setting $\theta=0$ and $L=0$ and taking the small $\phi$ limit in the asymptotic solution (4.7)-(4.9). Thus we have

$$
\Psi^{(0)}=\Phi^{(0)}=\frac{1}{2}, \quad \mathcal{Y}_{m}^{(0)}=\frac{1}{m^{2}-1}, \quad \mathcal{X}_{m}^{(0)}=-\frac{m^{2}}{3}, \quad \mathbb{C}_{a}^{(2)}=4(-1)^{a} .
$$

We can obtain the higher order terms by solving the system as follows. By inspecting the equations (5.17) and their perturbative expansions, one realizes that, to any order, the system can be solved in this schematic way: $\Psi^{(k)}-\Phi^{(k)} \rightarrow \mathcal{Y}_{m}^{(k)} \rightarrow \mathcal{X}_{m}^{(k)} \rightarrow \Psi^{(k)}+\Phi^{(k)} \rightarrow$ $\mathbb{C}_{a}^{(k)}$, provided the order $k-1$ functions are known. In the final step of computing $\mathbb{C}_{a}^{(k)}$ (5.20) two kinds of contributions must be distinguished. On the one hand, there is $\Delta_{\text {conv }}$ which is originated in from the convolutions with the Y-functions in (5.19). On the other hand we have contributions from the explicit functions in (5.12) which can be expanded to

\footnotetext{
${ }^{15}$ The different choice in the $\mathcal{Y}_{m}$ expansion is to have similar recurrent equations to those of $\mathcal{X}_{m}$.
} 
any order independently of the Y-system solution. These give

$$
\begin{aligned}
a^{4}\left[\frac{z_{0}^{[-a]}}{z_{0}^{[+a]}}\right]^{2} F(a, g)^{2} & =a^{4}\left(\frac{a-\sqrt{a^{2}+16 g^{2}}}{a+\sqrt{a^{2}+16 g^{2}}}\right)^{2} e^{i\left(\Phi\left(z_{0}^{[+a]}\right)-\Phi\left(z_{0}^{[-a]}\right)+\Phi\left(1 / z_{0}^{[-a]}\right)-\Phi\left(1 / z_{0}^{[+a]}\right)\right)}(\mathrm{F} .8 \\
& =16 g^{4}\left[1+\left(\pi^{2}-\frac{6}{a^{2}}\right) \frac{8 g^{2}}{3}+\left(7 \pi^{4}-\frac{150 \pi^{2}}{a^{2}}+\frac{630}{a^{4}}\right) \frac{16 g^{4}}{45}+\mathcal{O}\left(g^{6}\right)\right]
\end{aligned}
$$

where we have used that $z_{0}^{[ \pm a]}$ are given by $(2.16)$ at $q=0$. We kept the first 3 loop orders only.

\section{F.1 Small $\phi$ solution at 2-loops}

Let us now solve for the small $\phi$ Y-functions to the next to leading order in the coupling expansion. We refer to this as the 2-loop order computation because this order gives rise to a correction $\mathcal{O}\left(g^{4}\right)$ to the energy $\mathcal{E}$. Note however, that the Y-functions corresponding to this order are $\mathcal{O}\left(g^{2}\right)$.

We start with (5.15). Using the leading order of $\mathbb{C}_{a}$ and the expression for $K_{y, a}$,

$$
\Phi-\Psi \approx \sum_{a} \frac{16(-1)^{a} g^{2} \sqrt{4 g^{2}-u^{2}}}{\left(a^{2}+4 u^{2}\right)} .
$$

Given that fermionic Y-functions are defined in the interval $(-2 g, 2 g)$, this difference is essentially $\mathcal{O}\left(g^{3}\right)$. Thus, it will be convenient to use the variable $\tilde{u}=u /(2 g)$ that runs between -1 and 1 . We then obtain

$$
\Phi(2 g \tilde{u})-\Psi(2 g \tilde{u})=32 g^{3} \sqrt{1-\tilde{u}^{2}} \sum_{a=1}^{\infty} \frac{(-1)^{a}}{a^{2}}+\mathcal{O}\left(g^{5}\right)=-\frac{8 \pi^{2} g^{3}}{3} \sqrt{1-\tilde{u}^{2}}+\mathcal{O}\left(g^{5}\right) .
$$

This indicates that fermionic convolutions do not contribute to $\mathcal{X}_{n}^{(1)}$ or $\mathcal{Y}_{n}^{(1)}$ Then, we have

$$
\mathcal{Y}_{m}^{(1)}-s * I_{m, n} \mathcal{Y}_{n}^{(1)} \frac{\left(n^{2}-1\right)}{n^{2}}=0 .
$$

The same recurrence equations, thought with different rhs's, will repeatedly appear in the weak coupling expansion of the small angle limit TBA equations. In F.3 we present the resolvent of the corresponding recurrence operator (demanding $\mathcal{Y}_{1}^{(1)}=0$ and that $\mathcal{Y}_{m}^{(1)}$ remains bounded as $m \rightarrow \infty)$. In this particular case, we solve (F.11) with $\mathcal{Y}_{m}^{(1)}=0$.

The recurrence equation that $\mathcal{X}_{m}^{(1)}$ satisfies is non-homogeneous. It has a non-vanishing r.h.s. because of the term $\pi s \mathbb{C}_{m}$ in (5.18),

$$
\mathcal{X}_{m}^{(1)}-s * I_{m, n} \mathcal{X}_{n}^{(1)} \frac{\left(n^{2}-1\right)}{n^{2}}=4 \pi(-1)^{m} s
$$

In Fourier space this becomes

$$
2 \cosh \frac{w}{2} \widetilde{\mathcal{X}}_{m}^{(1)}-\frac{m(m-2)}{(m-1)^{2}} \widetilde{\mathcal{X}}_{m-1}^{(1)}-\frac{m(m+2)}{(m+1)^{2}} \widetilde{\mathcal{X}}_{m+1}^{(1)}=4 \pi(-1)^{m},
$$


which can be solved using the resolvent of F.3. Here we write down only the component we need

$$
\widetilde{\mathcal{X}}_{2}^{(1)}=-\frac{8}{3} \pi\left[2 \cosh \frac{w}{2} \log \left(1+e^{-\frac{|w|}{2}}\right)-1-e^{-\frac{|w|}{2}}\right] .
$$

Now, from (5.16), we have

$$
\Phi^{(1)}+\Psi^{(1)}=-\frac{3}{2} \mathcal{s} * \mathcal{X}_{2}^{(1)}+8 \pi(-1)^{n} \mathcal{s} * K_{n-1}-4 \pi(-1)^{a} K_{a},
$$

where we have used

$$
\mathcal{R}_{2 b}^{(01)}(u, 0)=K_{b-1}(u)+\frac{8 g^{2}}{b^{2}} K_{1}(u)+O\left(g^{4}\right) .
$$

To compute the convolutions we go to Fourier space and get

$$
\widetilde{\Phi}^{(1)}+\widetilde{\Psi}^{(1)}=4 \pi \log \left(1+e^{-|w| / 2}\right),
$$

and then

$$
\Phi^{(1)}+\Psi^{(1)}=\frac{1}{u^{2}}\left(1-\frac{2 \pi u}{\sinh (2 \pi u)}\right) .
$$

To compute convolution with this fermionic Y-functions $(\Phi$ and $\Psi)$ we shall use the aforementioned $\tilde{u}$, at the expense of introducing a $g$-dependence which has to be expanded as

$$
\Phi^{(1)}(2 g \tilde{u})+\Psi^{(1)}(2 g \tilde{u})=\frac{2 \pi^{2}}{3}-\frac{56 \pi^{4}}{45} g^{2} \tilde{u}^{2}+\mathcal{O}\left(g^{4}\right) .
$$

For the next to leading order it is enough to keep only the constant term. Note, however, that the second term will also contribute to the next to next to leading order.

We now compute

$$
\Delta_{\text {conv }}=\mathcal{R}_{a 1}^{(10)} \hat{*} \log 2 \Psi-\mathcal{B}_{a 1}^{(10)} \hat{*} \log 2 \Phi+\left[\mathcal{R}_{a b}^{(10)}+\mathcal{B}_{a, b-2}^{(10)}\right] * \log \frac{1+\mathcal{Y}_{b}}{1+\frac{1}{b^{2}-1}} .
$$

and we find to the leading order

$$
\Delta_{\text {conv }}=\frac{2 \pi^{2} g^{2}}{3}+\mathcal{O}\left(g^{4}\right) .
$$

We are now in the position to evaluate $\mathbb{C}_{a}$ at the 2-loop order. Inserting (F.21) and (F.8) into (5.20) we find

$$
\mathbb{C}_{a}=4(-1)^{a} g^{2}+8(-1)^{a}\left[\pi^{2}-\frac{4}{a^{2}}\right] g^{4}+\mathcal{O}\left(g^{6}\right),
$$

\section{F.2 Small $\phi$ solution at 3-loops}

Convolutions with fermionic Y-functions $(\Phi$ and $\Psi$ ) start contributing to the next to next to leading order. We have already obtained the difference of fermionic Y-functions in (F.10). For the convolution needed in (5.17) we get

$$
s \hat{*} \log \frac{\Psi}{\Phi} \simeq \frac{16 \pi^{2} g^{3}}{3} s(u-2 g \tilde{u}) \hat{*} \sqrt{1-\tilde{u}^{2}} \simeq \frac{16 \pi^{3} g^{4}}{3} s(u) .
$$


Thus, from (5.17) using $\mathcal{Y}_{m} \simeq \frac{1}{m^{2}-1}\left(1+g^{4} \mathcal{Y}_{m}^{(2)}+\ldots\right)$ we obtain

$$
\mathcal{Y}_{m}^{(2)}-s * I_{m, n} \mathcal{Y}_{n}^{(2)} \frac{\left(n^{2}-1\right)}{n^{2}}=-\frac{16 \pi^{3}}{3} \delta_{m, 2} s
$$

This recursive equation in Fourier space is

$$
2 \cosh \frac{w}{2} \widetilde{\mathcal{Y}}_{m}^{(2)}-\frac{m(m-2)}{(m-1)^{2}} \widetilde{\mathcal{Y}}_{m-1}^{(2)}-\frac{m(m+2)}{(m+1)^{2}} \widetilde{\mathcal{Y}}_{m+1}^{(2)}=-\frac{16 \pi^{3}}{3} \delta_{m, 2},
$$

for which we find the solution

$$
\widetilde{\mathcal{Y}}_{m}^{(2)}=-\frac{16 \pi^{3}}{3} \frac{m}{m^{2}-1} e^{-\frac{1}{2} m|w|}\left(\cosh \frac{w}{2}+m \sinh \frac{|w|}{2}\right),
$$

valid for $m \geq 2$, otherwise $\widetilde{\mathcal{Y}}_{m}^{(2)}$ vanishes.

Next, we consider the equation for $\mathcal{X}_{m}$. Recall that we are expanding it as $\mathcal{X}_{m}=$ $-\frac{m^{2}}{3}+g^{2} \mathcal{X}_{m}^{(1)}+g^{4} \mathcal{X}_{m}^{(2)}+\ldots$ Here, $\mathcal{X}_{m}^{(1)}$ is the solution to (F.12), for which we have only quoted $\mathcal{X}_{2}^{(1)}$. This however does not contribute to the equation for $\mathcal{X}_{m}^{(2)}$. For the latter we get the usual recurrence equation. In the r.h.s. there are contributions from the solution $\mathcal{Y}_{m}^{(2)}, \mathbb{C}_{m}$ and the fermionic convolution. In Fourier space the recurrence equations are

$$
\begin{aligned}
2 \cosh \frac{w}{2} \widetilde{\mathcal{X}}_{m}^{(2)}-\frac{m(m-2)}{(m-1)^{2}} \widetilde{\mathcal{X}}_{m-1}^{(2)}-\frac{m(m+2)}{(m+1)^{2}} \widetilde{\mathcal{X}}_{m+1}^{(2)}= & \frac{2}{3} \cosh \frac{w}{2} \widetilde{\mathcal{Y}}_{m}^{(2)} \\
& +(-1)^{m} 8\left[\pi^{3}-\frac{4 \pi}{m^{2}}\right]-\frac{8}{9} \pi^{3} \delta_{m, 2}
\end{aligned}
$$

where $\widetilde{\mathcal{Y}}_{m}^{(2)}$ is the the solution (F.26). This equation was also solved using the resolvent presented in F.3. We write only $\widetilde{\mathcal{X}}_{2}^{(2)}$,

$$
\begin{aligned}
\widetilde{\mathcal{X}}_{2}^{(2)}= & -\frac{128 \pi}{3}\left[\operatorname{Li}_{2}\left(-e^{-|w| / 2}\right) \sinh \frac{|w|}{2}+\operatorname{Li}_{3}\left(-e^{-|w| / 2}\right) \cosh \frac{w}{2}+1\right] \\
& -\frac{8 \pi^{3}}{27}\left(5 e^{-3|w| / 2}-9 e^{-|w| / 2}\right)-\frac{16 \pi^{3}}{3}\left[2 \cosh \frac{w}{2} \log \left(e^{-|w| / 2}+1\right)-1\right] .
\end{aligned}
$$

which is the only component that enters in the fermionic TBA equations. Then, from the fermionic Y-functions, we get

$$
\begin{aligned}
\Phi^{(2)}+\Psi^{(2)}= & 2 \pi s *\left[8 \pi^{2}(-1)^{n} K_{n-1}+\frac{32(-1)^{n}}{n^{2}}\left(K_{1}-K_{n-1}\right)\right] \\
& -8 \pi(-1)^{a}\left[\pi^{2}-\frac{4}{a^{2}}\right] K_{a}-\frac{3}{2} s * \mathcal{X}_{2}^{(2)}-\frac{1}{2} s * \mathcal{Y}_{2}^{(2)}
\end{aligned}
$$

We compute the convolutions in Fourier space,

$$
\widetilde{\Phi}^{(2)}+\widetilde{\Psi}^{(2)}=\frac{4}{3} \pi^{3} e^{-|w|}+8 \pi^{3} \log \left(1+e^{-|w| / 2}\right)+32 \pi \operatorname{Li}_{3}\left(-e^{-|w| / 2}\right),
$$

and then

$$
\Phi^{(2)}+\Psi^{(2)}=\frac{4 \pi^{2}}{3\left(1+u^{2}\right)}+\frac{2 \pi^{2}}{u^{2}}\left(1-\frac{2 \pi u}{\sinh (2 \pi u)}\right)+\frac{2}{u^{4}}\left(1-\frac{2 \pi u}{\sinh (2 \pi u)}-\frac{2 \pi^{2} u^{2}}{3}\right) .
$$


Evaluating for $u=2 g \tilde{u}$ we obtain for the leading weak coupling limit

$$
\Phi^{(2)}(2 g \tilde{u})+\Psi^{(2)}(2 g \tilde{u})=\frac{4 \pi^{2}}{3}+\frac{32 \pi^{4}}{45}+\mathcal{O}\left(g^{2}\right)
$$

Now we have to compute up to the $\mathcal{O}\left(g^{4}\right)$ convolutions in (5.19). If we use ${ }^{16}$

$$
\begin{aligned}
& \mathcal{R}_{a 1}^{(10)}(u, v)+\mathcal{B}_{a 1}^{(10)}(u, v)=K_{a}(u, v) \\
& \mathcal{R}_{a 1}^{(10)}(u, v)-\mathcal{B}_{a 1}^{(10)}(u, v)=\hat{K}_{a, y}(u, v)=K\left(u+i \frac{a}{2}, v\right)-K\left(u-i \frac{a}{2}, v\right)
\end{aligned}
$$

we can re-write the fermionic convolutions in the TBA equation (5.19) as

$$
2 \mathcal{R}_{a 1}^{(10)} \hat{*} \log \Psi-2 \mathcal{B}_{a 1}^{(10)} \hat{*} \log \Phi=K_{a} \hat{*} \log \frac{\Psi}{\Phi}+K_{a, y} \hat{*} \log \Psi \Phi
$$

It is important to recall that we need only the $u \rightarrow 0$ limit of this. For the order $g^{4}$ of the first term in (F.35) we get

$$
\frac{16 \pi^{3} g^{4}}{3} K_{a}(0)=\frac{32 \pi^{2} g^{4}}{3 a}
$$

The second term in (F.35) is

$$
\begin{aligned}
K_{a, y} \hat{*} \log \Psi \Phi= & \int_{-2 g}^{2 g} d v K_{a, y}(0, v)\left[\log (-4)+2 g^{2}\left(\Psi^{(1)}(v)+\Phi^{(1)}(v)\right)\right. \\
& \left.-\left(\Psi^{(1)}(v)+\Phi^{(1)}(v)\right)^{2} g^{4}+2\left(\Psi^{(2)}(v)+\Phi^{(2)}(v)\right) g^{4}+\cdots\right],
\end{aligned}
$$

where its $g^{4}$ order is

$$
-\frac{56 \pi^{4} g^{4}}{45} \int_{-1}^{1} d \tilde{v} \frac{2 \tilde{v}^{2}}{\pi \sqrt{1-\tilde{v}^{2}}}-\frac{9 \pi^{4} g^{4}}{4}+2\left(\frac{4 \pi^{2}}{3}+\frac{32 \pi^{4}}{45}\right) g^{4}=-\frac{12 \pi^{4} g^{4}}{45}+\frac{8 \pi^{2} g^{4}}{3}
$$

Thus, the total $g^{4}$ order of (F.35) is

$$
\frac{32 \pi^{2} g^{4}}{3 a}+\frac{8 \pi^{2} g^{4}}{3}-\frac{12 \pi^{4} g^{4}}{45} .
$$

For the remaining convolution in $(5.19), 2\left[\mathcal{R}_{a b}^{(10)}+\mathcal{B}_{a, b-2}^{(10)}\right] * \log \left(1+\mathcal{Y}_{b}\right)$ we use

$$
\mathcal{R}_{a b}^{(10)}(0, v)+\mathcal{B}_{a, b-2}^{(10)}(0, v)=K_{b-1}(v)+\sum_{j=-\frac{a-1}{2}}^{\frac{a-3}{2}} K_{b+2 j}+\mathcal{O}\left(g^{2}\right)
$$

We go to Fourier space, where the term order $g^{4}$ is

$$
2\left[\widetilde{K}_{b-1}+\sum_{j=-\frac{a-1}{2}}^{\frac{a-3}{2}} \widetilde{K}_{b+2 j}\right] \frac{\widetilde{\mathcal{Y}}_{b}^{(2)}}{b^{2}}=-\frac{8 \pi^{3}}{3} e^{-|w|}-\frac{16 \pi^{3}}{3} \frac{a-1}{a} e^{-\frac{a|w|}{2}} .
$$

\footnotetext{
${ }^{16}$ Note the difference with $\hat{K}_{y, a}$ defined in (F.3).
} 
We Fourier transform back and evaluate for $u \rightarrow 0$ and we get

$$
\left.2\left[K_{b-1}+\sum_{j=-\frac{a-1}{2}}^{\frac{a-3}{2}} K_{b+2 j}\right] * \frac{\mathcal{Y}_{b}^{(2)}}{b^{2}}\right|_{u=0}=\frac{32 \pi^{2}}{3 a^{2}}-\frac{32 \pi^{2}}{3 a}-\frac{8 \pi^{2}}{3} .
$$

Thus, for $\Delta_{\text {conv }}$ up the 3-loop order we have

$$
\Delta_{\text {conv }}=\frac{2 \pi^{2} g^{2}}{3}+\left[-\frac{6 \pi^{4}}{45}+\frac{16 \pi^{2}}{3 a^{2}}\right] g^{4}+\mathcal{O}\left(g^{6}\right) .
$$

Which, together with (F.8), gives rise to

$$
\mathbb{C}_{a}=4(-1)^{a} g^{2}+8(-1)^{a}\left[\pi^{2}-\frac{4}{a^{2}}\right] g^{4}+16(-1)^{a}\left[\frac{\pi^{4}}{3}-\frac{4 \pi^{2}}{a^{2}}+\frac{20}{a^{4}}\right] g^{6}+\mathcal{O}\left(g^{8}\right) .
$$

\section{F.3 Recurrence resolvent}

For solving perturbatively the small $\phi$ TBA equations we are faced with certain recurrence equations, all of same form but with different inhomogeneities. They can be solved in terms of the resolvent $\hat{\chi}_{n}{ }^{m}=f(m, n)$,for $n \geq m$ and $\hat{\chi}_{n}{ }^{m}=f(n, m)$ for $n<m$ with

$$
f(m, n)=-2 \sqrt{2} \frac{\left(\cosh \frac{w}{2}+n \sinh \frac{|w|}{2}\right)\left(\cosh \frac{w}{2} \sinh \frac{m|w|}{2}-m \sinh \frac{|w|}{2} \cosh \frac{m w}{2}\right)}{m n(\cosh w-1)^{3 / 2}} e^{-\frac{n|w|}{2}}
$$

This resolvent $\hat{\chi}_{n}^{m}$ is the solution to

$$
2 \cosh \frac{w}{2} \frac{n^{2}}{n^{2}-1} \hat{\chi}_{n}^{m}-\hat{\chi}_{n-1}^{m}-\hat{\chi}_{n+1}^{m}=\delta_{n}^{m} .
$$

This can be transformed into our recurrence relations by setting $\chi_{m}=\frac{m^{2}}{m^{2}-1} \hat{\chi}$.

\section{References}

[1] A.M. Polyakov, Gauge Fields as Rings of Glue, Nucl. Phys. B 164 (1980) 171 [INSPIRE].

[2] N. Drukker, D.J. Gross and H. Ooguri, Wilson loops and minimal surfaces, Phys. Rev. D 60 (1999) 125006 [hep-th/9904191] [INSPIRE].

[3] G. Korchemsky and A. Radyushkin, Infrared factorization, Wilson lines and the heavy quark limit, Phys. Lett. B 279 (1992) 359 [hep-ph/9203222] [INSPIRE].

[4] T. Becher and M. Neubert, Infrared singularities of QCD amplitudes with massive partons, Phys. Rev. D 79 (2009) 125004 [Erratum ibid. D 80 (2009) 109901] [arXiv:0904.1021] [INSPIRE].

[5] D. Correa, J. Henn, J. Maldacena and A. Sever, The cusp anomalous dimension at three loops and beyond, JHEP 05 (2012) 098 [arXiv:1203.1019] [INSPIRE].

[6] N. Beisert, B. Eden and M. Staudacher, Transcendentality and Crossing, J. Stat. Mech. 0701 (2007) P01021 [hep-th/0610251] [INSPIRE].

[7] A. Pineda, The Static potential in $N=4$ supersymmetric Yang-Mills at weak coupling, Phys. Rev. D 77 (2008) 021701 [arXiv:0709.2876] [InSPIRE]. 
[8] D. Correa, J. Henn, J. Maldacena and A. Sever, An exact formula for the radiation of a moving quark in $N=4$ super Yang-Mills, JHEP 06 (2012) 048 [arXiv:1202.4455] [INSPIRE].

[9] B. Fiol, B. Garolera and A. Lewkowycz, Exact results for static and radiative fields of a quark in $N=4$ super Yang-Mills, JHEP 05 (2012) 093 [arXiv:1202.5292] [INSPIRE].

[10] D.E. Berenstein, J.M. Maldacena and H.S. Nastase, Strings in flat space and pp waves from $N=4$ super Yang-Mills, JHEP 04 (2002) 013 [hep-th/0202021] [INSPIRE].

[11] N. Drukker and S. Kawamoto, Small deformations of supersymmetric Wilson loops and open spin-chains, JHEP 07 (2006) 024 [hep-th/0604124] [INSPIRE].

[12] N. Beisert et al., Review of AdS/CFT Integrability: An Overview, Lett. Math. Phys. 99 (2012) 3 [arXiv: 1012.3982] [INSPIRE].

[13] N. Beisert, The SU(2|2) dynamic S-matrix, Adv. Theor. Math. Phys. 12 (2008) 945 [hep-th/0511082] [INSPIRE].

[14] D.M. Hofman and J.M. Maldacena, Reflecting magnons, JHEP 11 (2007) 063 [arXiv:0708.2272] [INSPIRE].

[15] D. Correa and C. Young, Reflecting magnons from D7 and D5 branes, J. Phys. A 41 (2008) 455401 [arXiv: 0808.0452] [INSPIRE].

[16] D.H. Correa, V. Regelskis and C.A. Young, Integrable achiral D5-brane reflections and asymptotic Bethe equations, J. Phys. A 44 (2011) 325403 [arXiv:1105.3707] [InSPIRE].

[17] S. Ghoshal and A.B. Zamolodchikov, Boundary S matrix and boundary state in two-dimensional integrable quantum field theory, Int. J. Mod. Phys. A 9 (1994) 3841 [Erratum ibid. A 9 (1994) 4353] [hep-th/9306002] [InSPIRE].

[18] A. LeClair, G. Mussardo, H. Saleur and S. Skorik, Boundary energy and boundary states in integrable quantum field theories, Nucl. Phys. B 453 (1995) 581 [hep-th/9503227] [INSPIRE].

[19] K. Zarembo, Supersymmetric Wilson loops, Nucl. Phys. B 643 (2002) 157 [hep-th/0205160] [INSPIRE].

[20] N. Beisert and M. Staudacher, Long-range PSU $(2,2 \mid 4)$ Bethe Ansatze for gauge theory and strings, Nucl. Phys. B 727 (2005) 1 [hep-th/0504190] [INSPIRE].

[21] D. Volin, Minimal solution of the AdS/CFT crossing equation, J. Phys. A 42 (2009) 372001 [arXiv: 0904.4929] [INSPIRE].

[22] P. Vieira and D. Volin, Review of AdS/CFT Integrability, Chapter III.3: The Dressing factor, Lett. Math. Phys. 99 (2012) 231 [arXiv:1012.3992] [INSPIRE].

[23] Z. Bajnok, L. Palla and G. Takács, Finite size effects in quantum field theories with boundary from scattering data, Nucl. Phys. B 716 (2005) 519 [hep-th/0412192] [INSPIRE].

[24] N. Drukker, Integrable Wilson loops, arXiv:1203.1617.

[25] N. Beisert, The Analytic Bethe Ansatz for a Chain with Centrally Extended SU(2|2) Symmetry, J. Stat. Mech. (2007) P01017 [nlin/0610017].

[26] N. Beisert, R. Hernandez and E. Lopez, A Crossing-symmetric phase for $A d S_{5} \times S^{5}$ strings, JHEP 11 (2006) 070 [hep-th/0609044] [inSPIRE].

[27] S. Yamaguchi, Wilson loops of anti-symmetric representation and D5-branes, JHEP 05 (2006) 037 [hep-th/0603208] [INSPIRE]. 
[28] D.M. Hofman and J.M. Maldacena, Giant Magnons, J. Phys. A 39 (2006) 13095 [hep-th/0604135] [INSPIRE].

[29] G. Arutyunov and S. Frolov, On String S-matrix, Bound States and TBA, JHEP 12 (2007) 024 [arXiv:0710.1568] [INSPIRE].

[30] D. Correa and C. Young, Finite size corrections for open strings/open chains in planar AdS/CFT, JHEP 08 (2009) 097 [arXiv:0905.1700] [INSPIRE].

[31] A. Zamolodchikov, Thermodynamic Bethe ansatz in relativistic models. Scaling three state potts and Lee-Yang models, Nucl. Phys. B 342 (1990) 695 [inSPIRE].

[32] G. Arutyunov and S. Frolov, String hypothesis for the $A d S_{5} \times S^{5}$ mirror, JHEP 03 (2009) 152 [arXiv:0901.1417] [INSPIRE].

[33] C.-N. Yang and C. Yang, Thermodynamics of one-dimensional system of bosons with repulsive delta function interaction, J. Math. Phys. 10 (1969) 1115 [INSPIRE].

[34] D. Bombardelli, D. Fioravanti and R. Tateo, Thermodynamic Bethe Ansatz for planar AdS/CFT: A Proposal, J. Phys. A 42 (2009) 375401 [arXiv:0902.3930] [INSPIRE].

[35] G. Arutyunov and S. Frolov, Thermodynamic Bethe Ansatz for the $A d S_{5} \times S^{5}$ Mirror Model, JHEP 05 (2009) 068 [arXiv: 0903.0141] [INSPIRE].

[36] N. Gromov, V. Kazakov, A. Kozak and P. Vieira, Exact Spectrum of Anomalous Dimensions of Planar $N=4$ Supersymmetric Yang-Mills Theory: TBA and excited states, Lett. Math. Phys. 91 (2010) 265 [arXiv:0902 .4458] [INSPIRE].

[37] N. Gromov, V. Kazakov and P. Vieira, Exact Spectrum of Anomalous Dimensions of Planar $N=4$ Supersymmetric Yang-Mills Theory, Phys. Rev. Lett. 103 (2009) 131601 [arXiv: 0901.3753] [INSPIRE].

[38] N. Gromov, V. Kazakov, A. Kozak and P. Vieira, Exact Spectrum of Anomalous Dimensions of Planar $N=4$ Supersymmetric Yang-Mills Theory: TBA and excited states, Lett. Math. Phys. 91 (2010) 265 [arXiv:0902.4458] [INSPIRE].

[39] P. Dorey and R. Tateo, Excited states by analytic continuation of TBA equations, Nucl. Phys. B 482 (1996) 639 [hep-th/9607167] [INSPIRE].

[40] V.V. Bazhanov, S.L. Lukyanov and A.B. Zamolodchikov, Integrable quantum field theories in finite volume: Excited state energies, Nucl. Phys. B 489 (1997) 487 [hep-th/9607099] [INSPIRE].

[41] S. Frolov and R. Suzuki, Temperature quantization from the TBA equations, Phys. Lett. B 679 (2009) 60 [arXiv:0906.0499] [inSPIRE].

[42] G. Arutyunov and S. Frolov, Simplified TBA equations of the $A d S_{5} \times S^{5}$ mirror model, JHEP 11 (2009) 019 [arXiv:0907.2647] [INSPIRE].

[43] G. Arutyunov, S. Frolov and R. Suzuki, Exploring the mirror TBA, JHEP 05 (2010) 031 [arXiv: 0911.2224] [INSPIRE].

[44] N. Gromov, V. Kazakov, S. Leurent and D. Volin, Solving the AdS/CFT Y-system, JHEP 07 (2012) 023 [arXiv:1110.0562] [INSPIRE].

[45] O. Aharony, O. Bergman, D.L. Jafferis and J. Maldacena, $N=6$ superconformal Chern-Simons-matter theories, M2-branes and their gravity duals, JHEP 10 (2008) 091 [arXiv:0806.1218] [INSPIRE]. 
[46] N. Gromov and P. Vieira, The all loop AdS4/CFT3 Bethe ansatz, JHEP 01 (2009) 016 [arXiv: 0807.0777] [INSPIRE].

[47] V. Forini, Quark-antiquark potential in AdS at one loop, JHEP 11 (2010) 079 [arXiv: 1009.3939] [INSPIRE].

[48] N. Drukker and V. Forini, Generalized quark-antiquark potential at weak and strong coupling, JHEP 06 (2011) 131 [arXiv: 1105.5144] [INSPIRE].

[49] N. Gromov, Y-system and Quasi-Classical Strings, JHEP 01 (2010) 112 [arXiv:0910.3608] [INSPIRE].

[50] N. Gromov and F. Levkovich-Maslyuk, Y-system, TBA and Quasi-Classical strings in $A d S_{4} \times C P^{3}, J H E P 06$ (2010) 088 [arXiv:0912.4911] [INSPIRE].

[51] N. Gromov, V. Kazakov and P. Vieira, Exact Spectrum of Planar $\mathcal{N}=4$ Supersymmetric Yang-Mills Theory: Konishi Dimension at Any Coupling, Phys. Rev. Lett. 104 (2010) 211601 [arXiv:0906.4240] [INSPIRE].

[52] L.F. Alday and J. Maldacena, Comments on gluon scattering amplitudes via AdS/CFT, JHEP 11 (2007) 068 [arXiv:0710.1060] [INSPIRE].

[53] N. Dorey, D.M. Hofman and J.M. Maldacena, On the Singularities of the Magnon S-matrix, Phys. Rev. D 76 (2007) 025011 [hep-th/0703104] [INSPIRE].

[54] G. Arutyunov and S. Frolov, The Dressing Factor and Crossing Equations, J. Phys. A 42 (2009) 425401 [arXiv:0904.4575] [INSPIRE].

[55] V. Kazakov, A.S. Sorin and A. Zabrodin, Supersymmetric Bethe ansatz and Baxter equations from discrete Hirota dynamics, Nucl. Phys. B 790 (2008) 345 [hep-th/0703147] [INSPIRE].

[56] Z. Tsuboi, Analytic Bethe ansatz and functional equations for Lie superalgebra $s l(r+1 \mid s+1)$, J. Phys. A 30 (1997) 7975 [arXiv:0911.5386] [InSPIRE].

[57] N. Beisert, V. Kazakov, K. Sakai and K. Zarembo, Complete spectrum of long operators in $N=4 S Y M$ at one loop, JHEP 07 (2005) 030 [hep-th/0503200] [INSPIRE].

[58] N. Gromov and V. Kazakov, Review of AdS/CFT Integrability, Chapter III.\%: Hirota Dynamics for Quantum Integrability, Lett. Math. Phys. 99 (2012) 321 [arXiv:1012.3996] [INSPIRE].

[59] C. Ahn, Z. Bajnok, D. Bombardelli and R.I. Nepomechie, TBA, NLO Lüscher correction and double wrapping in twisted AdS/CFT, JHEP 12 (2011) 059 [arXiv:1108.4914] [INSPIRE].

[60] M. de Leeuw and S.J. van Tongeren, The spectral problem for strings on twisted $A d S_{5} \times S^{5}$, Nucl. Phys. B 860 (2012) 339 [arXiv:1201.1451] [INSPIRE].

[61] A. Dekel and Y. Oz, Integrability of Green-Schwarz $\sigma$-models with Boundaries, JHEP 08 (2011) 004 [arXiv:1106.3446] [INSPIRE]. 